\title{
Ultrasonic Inspection of Drinking Water Mains
}

\section{Hector Hernandez Delgadillo}




\section{ULTRASONIC INSPECTION OF DRINKING WATER MAINS}

Hector Hernandez Delgadillo 



\section{ULTRASONIC INSPECTION OF DRINKING WATER MAINS}

\section{DISSERTATION}

to obtain

the degree of doctor at the Universiteit Twente, on the authority of the rector magnificus, prof.dr. T.T.M. Palstra, on account of the decision of the graduation committee

to be publicly defended on

Wednesday the $30^{\text {th }}$ of October 2019 at 12.45 p.m.

by

Hector Hernandez Delgadillo

born on the $1^{\text {st }}$ of May 1987

in Mexico City, Mexico 
This dissertation has been approved by:

supervisors

prof.dr.ir. R. Akkerman

prof.dr.ir. T. Tinga

co-supervisor

dr.ir. R. Loendersloot

Cover design: $\quad$ Photo taken by Doekle Yntema and cover designed by

Printed by: Gildeprint

ISBN:

$978-90-365-4853-3$

DOI: $\quad 10.3990 / 1.9789036548533$

(C) H. Hernandez Delgadillo, Leeuwarden, The Netherlands, 2019

The Netherlands. All rights reserved. No parts of this thesis may be reproduced, stored in a retrieval system or transmitted in any form or by any means without permission of the author. 


\section{Graduation Committee:}

\begin{tabular}{|c|c|c|}
\hline $\begin{array}{l}\text { Chairman / } \\
\text { secretary }\end{array}$ & prof.dr. G.P.M.R. Dewulf & University of Twente \\
\hline supervisors: & $\begin{array}{l}\text { prof.dr.ir. R. Akkerman } \\
\text { prof.dr.ir. T. Tinga }\end{array}$ & $\begin{array}{l}\text { University of Twente } \\
\text { University of Twente }\end{array}$ \\
\hline co-supervisor: & dr.ir R. Loendersloot & University of Twente \\
\hline $\begin{array}{l}\text { Committee } \\
\text { Members }\end{array}$ & $\begin{array}{l}\text { prof.dr.ir. A.G. Doree } \\
\text { prof.dr.ir. W. Steenbergen } \\
\text { prof.dr.ir. K. Keesman } \\
\text { prof.dr.ir. F.H.L.R. Clemens } \\
\text { prof.dr. A.J. Croxford }\end{array}$ & $\begin{array}{l}\text { University of Twente } \\
\text { University of Twente } \\
\text { Wageningen University } \\
\text { Delf University of Technology } \\
\text { University of Bristol }\end{array}$ \\
\hline
\end{tabular}



A mis padres 



\section{Summary}

The Dutch drinking water network comprises over 120 thousand kilometres of infrastructure. A large number of these pipes have exceeded their expected operational life-time, while other pipes can still operate for many more years. Judging which pipes have reached their end of operational life is a complex task. However, failing to replace the infrastructure on time (before rupture) can cause serious damage to society, to the nearby infrastructure and to the finances of the water utilities. Preventive maintenance policies can diminish the uncertainty of replacing the 'right' pipe, thus increasing the reliability of the drinking water network.

Condition-based strategies enhance the replacement decision-making. Establishing new strategies for maintenance policies is not a straightforward decision. Obviously, many factors have to be considered, including the cost of launching a condition-based system versus the cost of repairing a failed pipe. With a very complex infrastructure, both below ground and above ground, it is very likely that a failed pipe will cause significant damage to other constructions. And amongst many other negative consequences, the image of a water utility can be greatly affected if its network is unreliable.

The Dutch water utilities have joined a scientific division with the purpose of advancing the reliability of their infrastructure. This is the Smart Water Grids platform, where synergy between academia, government and industrials (the water utilities) is achieved. Within this platform, the knowledge towards a safer, smarter and more sustainable supply of drinking water is promoted from a scientific perspective. In this thesis, the possibility of using ultrasonic sensors for the inspection of drinking water pipes in an inline configuration is investigated. Two materials are studied: polyvinyl chloride (PVC) and asbestos cement (AC).

The study conducted for the development of inspection methodologies is divided into material types. Chapter 2 and Chapter 3 focus on cement-based pipes. Chapter 4 and Chapter 5 describe the advancement of the wave mixing technique for the inspection of PVC pipes.

Chapter 1 describes the motivation to develop inspection methodologies as well as the motivation for using ultrasonic sensors. Thereafter, Chapter 2 describes the degradation mechanisms that reduce the service life of cement-based pipes. A methodology for quantifying degradation levels in cement-based materials is proposed.

Chapter 3 bridges the gap between the laboratory environment and field operations. In collaboration with Acquaint B.V. (Inspection Company) and Brabant Water (drinking water utility) an inline inspection in an asbestos cement pipe in service was performed. Based on the developed methodology in Chapter 2, the degradation levels from the inspected trajectory are determined. 
Chapter 4 describes the wave mixing technique principle. The possibility of using a wider range of excitation frequencies of the incident waves and of reducing the complexity of sensor positioning were investigated.

In Chapter 5, the wave mixing technique is tested in a PVC pipe taken from service by Evides Waterbedrijf (Dutch drinking water utility). From this research, it is found that signal-wise the wave mixing technique can be used for the inspection of PVC pipes from the inside surface.

Chapter 6 brings together the knowledge gained throughout this thesis. It organizes the knowledge from previous work, the work from this thesis and future work. In Chapter 7, the conclusion of this research and some recommendations are provided. 


\section{Samenvatting}

Het Nederlandse drinkwaternetwerk bestaat uit meer dan 120 duizend kilometer infrastructuur. Een hoog aantal van deze leidingen zijn voorbij de verwachte operationele levensduur, terwijl andere leidingen nog vele jaren mee kunnen. Evalueren of een leiding het einde van de levensduur bereikt heeft is een complexe taak. Het niet tijdig vervangen van de infrastructuur, voordat er scheuren in komen, kan ernstige gevolgen hebben voor de samenleving en nabij gelegen infrastructuur, maar ook financiële gevolgen hebben voor waterbedrijven. Beleid omtrent het verrichten van preventief onderhoud kan de onzekerheid verkleinen rondom het vervangen van de 'juiste' gedeelte van de leiding, en daardoor de betrouwbaarheid van het drinkwater netwerk vergroten.

Vervangingsttrategieën gebaseerd op de werkelijke conditie van een leiding verbeteren het besluitnemingsproces om leidingen te vervangen. Het aannemen van nieuwe strategieën voor het onderhoud beleid is niet een simpel besluit. Meerdere factoren spelen mee en moeten in de overweging meegenomen worden, waaronder ook de kosten voor het oprichten van een conditie gebaseerdsysteem t.o.v. de kosten van het vervangen van een kapotte leiding. Met complexe infrastructuren, zowel boven- als ondergrond, is het zeer aannemelijk dat een gescheurde leiding significante schade kan veroorzaken bij andere bebouwingen. Andere negatieve consequenties zijn dat het imago van waterbedrijven aangetast word als het waternetwerk niet betrouwbaar is.

Nederlandse waterbedrijven hebben een wetenschapelijke samenwerkingzijn samengekomen in een Wetsus samenwerking met als doel vooruitgang in de betrouwbaarheid van de infrastructuur. Dit is het Smart Water Grids platform, waar samen gewerkt word tussen academia, overheid en waterbedrijven. Dit platform promoot vanuit de wetenschappelijjkekennis een veiliger, slimmer en duurzamere wijze van de bevoorrading van drinkwater. Dit promotieonderzoek wordt onderzocht of het gebruik van ultrasoon sensoren het inspecteren van de drinkwaterleidingen in een inline configuratie mogelijk maakt. De studie richt ziich voornamelijjk op twee verschillende soorten materialmen: polyvinylchloride (PVC) en asbestcement (AC).

Het verrichtte onderzoek voor het ontwikkelen van inspectie methodologieën is opgesplitst naar type materiaal. Hoofdstukken 2 en 3 leggen de focus op leidingen van cement. Hoofdstukken 4 en 5 beschrijven de vordering van de wavemixing techniek die gebruikt is voor de inspectie van de leidingen die van PVC gemaakt zijn.

Hoofdstuk1 beschrijft de onderbouwing om inspectie methodologieën te ontwikkelen en de motivatie voor het gebruik van ultrasoon sensoren. In Hoofdstuk 2 worden de degradatie mechanismen omschreven die de levensloop 
van cement leidingen reduceren. Een methodologie om het niveau van degradatie in materialen van cement te quantificeren wordt ook beschreven.

Hoofdstuk3 slaat een brug tussen laboratorium onderzoek en praktisch veldwerk. In samenwerking met Acquaint B.V. (inspectie bedrijf) en Brabant Water (waterbedrijf) is een inline inspectie uitgevoerd in een operationele betonnen leiding. Volgens de in hoofdstuk 2 omschreven methodologieis het niveau van degradatie in het geinspecteerde stuk bepaald.

Hoofdstuk4 omschrijft het principe van de wave mixing techniek. De mogelijkheid om gebruik te maken van een breder bereikvan excitatie-frequenties van de inkomende geluidsgolven en het reduceren van de complexiteit van de sensorpositionering is ook onderzocht en om de geluidspatronen te sturen.

In hoofdstuk 5, the wave mixing techniek is getest op een door een Evides Waterbedrijf (Nederlands waterbedrijf) verwijderde leiding van PVC. Uit dit onderzoek is gebleken dat de signaaltechnisch- de wave mixing techniek gebruikt kan worden voor de inspectie van de binnenkant van de PVC leidingen.

Hoofdstuk 6 brengt de kennis die is opgedaan tijdens deze scriptie tezamen. Het ordent de opgedane kennis van voorgaande studies, deze studie en voor toekomstig werk. In hoofdstuk 7 is de conclusie met aanbevelingen voor toekomstig onderzoek te vinden. 


\section{Contents}

Summary

ix

Samenvatting

xi

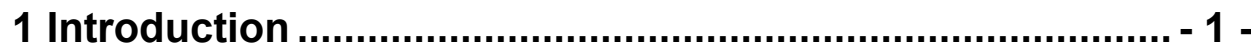

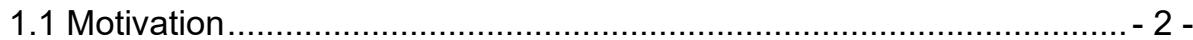

1.1.1 The Dutch drinking water network ......................................... - 3 -

1.1.2 Basic principle of ultrasonic testing (pulse-echo) ....................... - 4 -

1.1.3 Ultrasonic testing in drinking water pipes ................................ 7 -

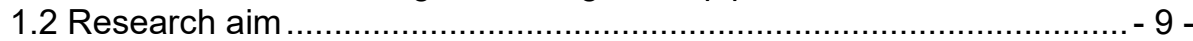

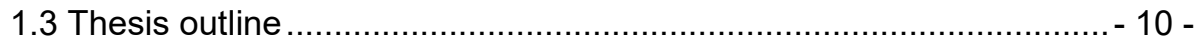

1.4 Publications ................................................................................. 11 -

2 A methodology based on pulse-velocity measurements to quantify the chemical degradation levels in thin mortar specimens .......................................................................... - 15 -

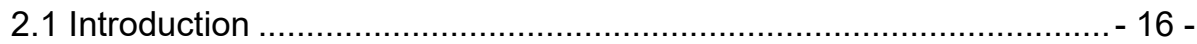

2.1.1 Condition assessment methods ........................................ 16 -

2.1.2 Ultrasonic testing in cement-based structures.......................... - 17 -

2.1.3 Cement hydration and main phases .................................... 18 -

2.1.4 Acidic deterioration ...................................................... 18 -

2.1.5 Calcium leaching .............................................................. 19 -

2.1.6 Effect of calcium content on the speed of sound.................... 20 -

2.1.7 Layers present in degraded cement ....................................... 20 -

2.2 Methods ............................................................................... 21 -

2.2.1 Production of specimens and preparation .............................. 21 -

2.2.2 Accelerated degradation .................................................. 22 -

2.2.3 Ultrasonic test .......................................................... 22 -

2.2.4 Signal processing ........................................................ 24 -

2.2.5 Noise removal ............................................................... 24 -

2.2.6 Analytic envelope (Hilbert transform) …………................... - 25 -

2.2.7 Estimation of degraded depth............................................ 26 -

2.2.8 Destructive testing and validation....................................... 27 -

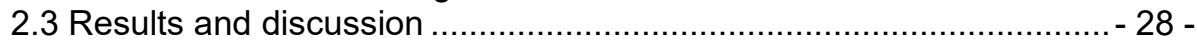

2.3.1 Estimated depth from calcium leaching ............................... 28 -

2.3.2 Validation from acidic deterioration ...................................... 32 -

2.3.3 Response at acoustic interfaces.......................................... 33 -

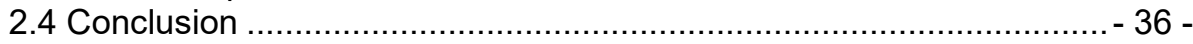




\section{Ultrasonic inline inspection of a cement-based drinking water} pipeline ............................................................................... 41 -

3.1 Introduction ................................................................................ 42 -

3.1.1 Commercially available inspection technologies .................... 42 -

3.1.2 Leaching depth in a degraded structure ............................... 42 -

3.2 Methodology ……................................................................... 43 -

3.2.1 Inspected trajectory ....................................................... 43 -

3.2.2 Automatic processing of ultrasonic signals............................. - 45 -

3.2.3 Visualization of extracted parameters ................................. 46 -

3.3 Results and discussion ............................................................ 47 -

3.3.1 Visualization of extracted parameters ................................. 47 -

3.3.2 Results of inspected section ............................................. 49 -

3.3.3 Sensitivity ................................................................. 56 -

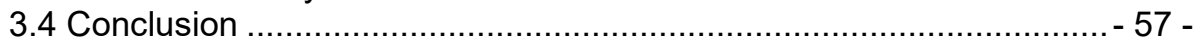

4 Steering the propagation direction of a non-linear acoustic wave in a solid material ........................................................ - 61 -

4.1 Introduction ................................................................... 62 -

4.2 Wave mixing theory ............................................................... 63 -

4.3 Methodology .............................................................................. 66 -

4.3.1 Analytical solution ......................................................... 66 -

4.3.2 Experimental set-up....................................................... 67 -

4.4 Results and discussion ......................................................... 72 -

4.4.1 Analytical results ........................................................... 72 -

4.4.2 Experimental results .................................................... 74 -

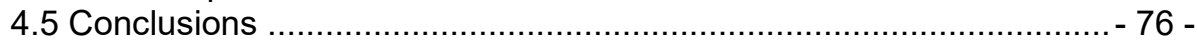

\section{Power spectral analysis of a one-sided non-collinear wave} mixing test in a PVC pipe ................................................. - 79 -

5.1 Introduction ........................................................................... 80

5.1.1 Polyvinyl chloride pipes ................................................. 81 -

5.1.2 The non-collinear wave mixing principle................................ 81 -

5.2 Methodology .......................................................................... 83 -

5.2.1 PVC samples ................................................................. 83 -

5.2.2 Longitudinal wave mode speed of sound ............................. - 84 -

5.2.3 Shear wave mode speed of sound ..................................... 85 -

5.2.4 Non-collinear wave mixing................................................ 88 -

5.2.5 Field test of PVC drinking water pipe ................................. 91 -

5.3 Results and discussion .............................................................. 94 -

5.3.1 Non-collinear wave mixing laboratory tests ........................... 94 -

5.3.2 Testing of PVC pipe.......................................................... 95 -

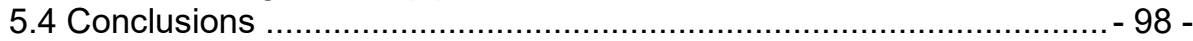

6 General Discussion.................................................. - 103 -

6.1 Initial problem overview ........................................................... 104 - 
6.2 Development of ultrasonic inspection methods for the drinking water pipes

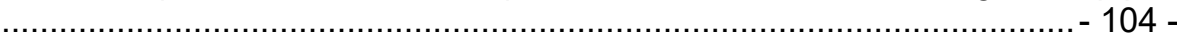

6.3 Summary on achieved research objectives ............................... 108 6.4 Outlook on inspection technologies and management of the drinking water network.................................................................................... 109 -

7 Conclusions and recommendations ............................... - 113 -

7.1 Conclusions ........................................................................ 114 -

7.2 Recommendations ............................................................ $115-$

7.2.1 Scientific recommendations ............................................... $115-$

7.2.2 General recommendations ....................................... 115 -

Acknowledgements ........................................................ - 117 -

About The Author................................................................ - 121 - 



\section{Chapter 1}

\section{Introduction}




\subsection{Motivation}

Drinking water supply systems have been in operation since the Roman Empire (27 B.C - 476 A.D). Lead pipes were used for the distribution of water within cities and were placed above ground level. By potential energy, the water was carried through the system to the users. The pipes were replaced when the flow rate in the households was extremely low due to clogging [1-3]. This was considered a replacement trigger. The cause of clogging was lime accumulation inside the tubes. Similarly, a broken pipe led to a low flow rate. However, the physics behind the failure mechanisms that led to pipe rupture were not known by ancient civilizations.

Only in the last two centuries has knowledge on the life cycle of drinking water systems been developed. The main reason for this is that the economy of many countries around the world grew at rates never experienced before [4]. Economic growth ultimately leads to a more complex infrastructure, for instance in the area of communications, energy and drinking water. In the early 1900s, the developments in water treatment led to higher production volumes of drinking water. In turn, the transmission and distribution networks of drinking water improved technologically [1, 4]. Some of these advancements were the introduction of materials with a high mechanical performance for the transmission pipelines, as these were laid underground. With such high mechanical performance, longer operational lifetime and greater reliability were expected.

With the expansion of urban areas, the risk of failure increased. Risk is defined as the product of the probability of failure and the consequence of a failed structure. The risk of damaging nearby infrastructure, blocking roads or interrupting the water supply to households increases. For example, in 2015 a hospital (VU Medical Centre) in Amsterdam had to be evacuated due to a pipe burst [5]. Some surgeries had to be cancelled and the medical instruments that were providing therapy to other patients were shut down. The roads around the area were flooded as well and many other private properties were damaged (see Figure 1.1a).
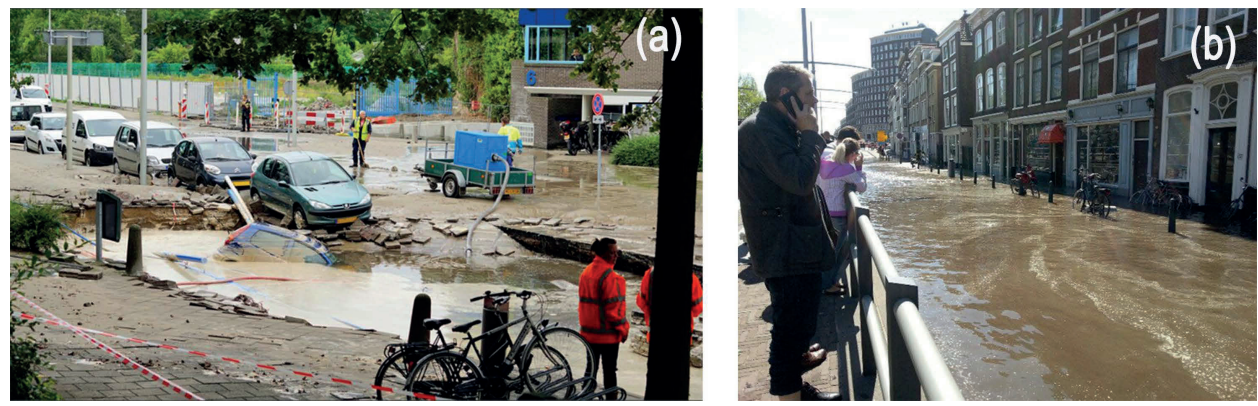

Figure 1.1

Damage to infrastructure: (a) in Amsterdam due to pipe burst in 2015 [8] and (b) road flooding in The Hague in 2016 [7]. 
The consequence of the failed pipe in this example is the most serious registered so far (in the Netherlands) as the lives of many patients were in danger. The estimated cost of the damage was approximately 35 million Euros [6]. In 2016 a pipe burst in the area of The Hague left the neighbouring zone without water supply for a couple of hours (see Figure 1.1b) [7]. The accident only damaged material infrastructure. With these two examples, it is clear that a pipe burst in a complex urban area can be rather catastrophic. It that sense, it is alarming that an incalculable number of pipes have reached their expected operational lifetime, as the drinking water supply network has been in operation for more than 50 years.

This raises the following questions: What is the condition of the network? Which pipes have the highest probability of failure? Which pipes should be replaced first? Many factors have to be considered in order to accurately answer these questions; accurately determining which pipes need to be replaced first is therefore a challenging task. An important factor is that the asset owners do not know precisely the location of all the pipes, or their characteristics. These characteristics may include wall thickness, year of manufacture, year of installation, mechanical properties, acoustic properties and quality. Even today, there is still no quality control on the manufactured pipes for the drinking water supply. The first step is to become familiar with the type of pipes that are laid underground. With this knowledge in mind, the focus in this thesis is given to understanding the degradation mechanisms of the pipes and the development of inspection technologies that can measure their current condition.

\subsubsection{The Dutch drinking water network}

The Dutch Water Act in the 1980s stipulated regulations that requested the suppliers of drinking water to ensure an operational and safe network [9]. In the events of calamities, the supply of drinking water must not be interrupted for long periods (maximum of 24 hours). Besides complying with minimum water quality standards, an action plan in case of breakdowns had to be developed. Natural disasters as well as pipe bursts were included in the plan. Furthermore, the reliability of the network had to be assessed.

The Dutch drinking water network has approximately 120 thousand $\mathrm{km}$ of pipes installed [10]. The different type of materials used in the network are depicted in Figure 1.2. The estimated cost of replacing the entire network at once is 30 billion Euros [11]. Approximately $80 \%$ of the network comprises two types of materials: asbestos cement $(\approx 30 \%)$ and polyvinyl chloride $(\approx 50 \%)$ pipes. In this thesis, the focus will be on these two materials.

Since 2009 many drinking water suppliers in the Netherlands are actively searching for new ways to minimize unforeseen pipe bursts as well as to maximize the efficiency of the replacement plans. Therefore, the Dutch drinking water suppliers, together with Wetsus research centre and Dutch universities, began to cooperate in a platform called 'Smart Water Grids'. In the Smart Water Grids platform, knowledge is generated in multiple scientific fields with the aim of better 
understanding the current state of the drinking water network and developing innovative inspection and prediction tools. The current academic and waterrelated partners involved in the smart water grids research theme are Vitens, Acquaint, Brabant Water, PWN, Wavin, Evides, Wageningen University and University of Twente.

Roy Visser's doctoral thesis [12] preceded the research projects in the Smart Water Grids theme. In Visser's doctoral research, a methodology to determine the remaining lifetime of PVC based on micro-indentation tests was developed [12]. Afterwards, Emiel Drenth continued the work of Visser, still outside the Smart Water Grid platform, and he developed a hardness test for the condition monitoring of PVC materials [13]. Both projects were motivated by pioneering a condition monitoring method for PVC infrastructure.

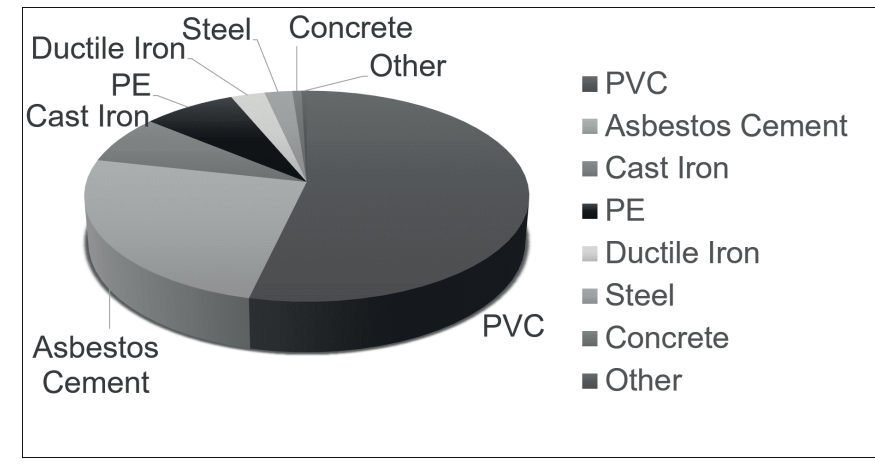

Figure 1.2

Distribution of pipes installed in the Netherlands in 2016. The total length of the Dutch drinking water network is $120,061 \mathrm{~km}[10]$.

In 2009 Andre Arsenio started the first doctoral project in the Smart Water Grids theme. Arsenio's research focused on understanding the failure mechanisms of PVC push-fit joints as well as on developing inspection and failure prediction tools for these joints [14]. In 2010 Mahmoud Ravanan started research to investigate the ultrasonic signature of chemical degradation in asbestos cement pipes and to develop an ultrasonic technique for the detection of ageing in PVC. Later Vaidotas Cicenas and Andriejus Demčenko took over his project [15].

The ARIEL inspection robot (prototype) is another example of the research performed in the Smart Water Grids platform. It was invented at Wetsus, with the financial contribution of KWR Watercycle Research Institute, and can pass autonomously through L-shaped bends [16]. It was designed to serve as a carrier for the technologies developed in the framework of the other projects.

\subsubsection{Basic principle of ultrasonic testing (pulse-echo)}

The ultrasonic testing principle is based on transmission and reflection of waves propagating in a medium. With the piezoelectric effect, it is possible to transmit 
and receive propagating acoustic waves. The piezoelectric effect is the ability of a material to convert electrical energy into mechanical energy and vice versa. If a voltage $V_{1}$ is applied to a piezoelectric material, the piezoelectric material will strain from thickness $D_{1}$ to thickness $D_{2}$. (see Figure 1.3a) and if a voltage $-V_{1}$ is applied to the piezoelectric material will strain from thickness $D_{1}$ to thickness $D_{2}$ as depicted in Figure 1.3b [17]. If the voltage $V_{1}$ is applied and removed at constant intervals, a periodic pressure fluctuation will be formed surrounding the surface of the piezoelectric material. The actuation of voltage into the piezoelectric element will generate the propagation of acoustic waves. In the reverse process (strained piezoelectric material) a voltage will be generated and, depending on the deformation direction, the voltage will be positive or negative. This effect is known as sensing.

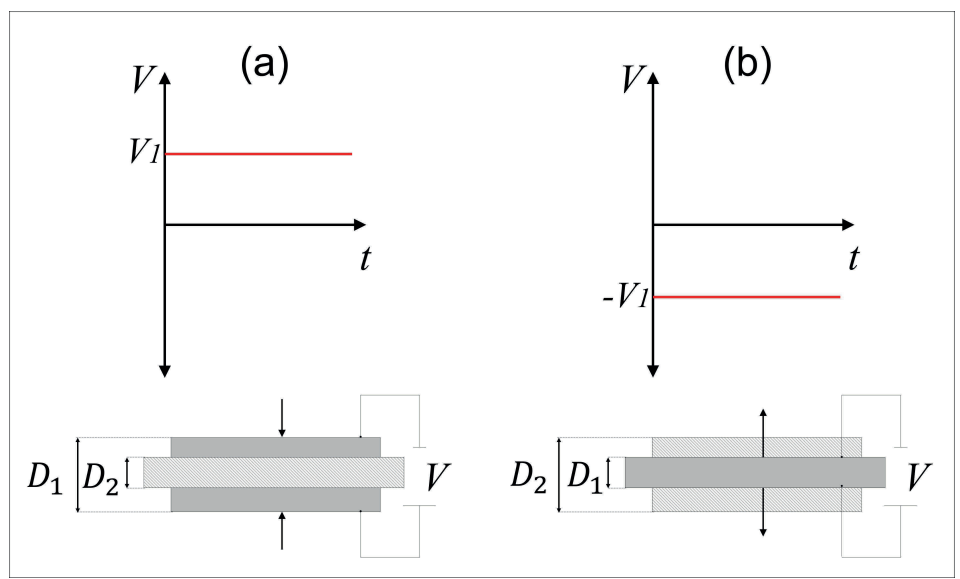

Figure 1.3

Piezoelectric effect in (a) compression generates voltage $V_{1}$ and (b) in tension generates a voltage $-V_{1}$.

The generated acoustic waves can propagate in two different modes. The first is when the particle movement (after applying a periodic voltage) moves in the direction of the propagation of the acoustic wave (see Figure 1.4a). This wave is called pressure or longitudinal wave.

The particles can also be excited in a direction perpendicular to the direction of the wave propagation, as shown in Figure 1.4b. Here, the particles are excited by shear forces. This type of wave propagation is called shear wave or transverse wave.

The linear behaviour of solids allows for waves to propagate due to its ability to return to its original state (both compression and shear). In fluids only compression/expansion is a reversible process. Shear forces are dissipative and thus, transverse waves cannot propagate in fluids. The material parameters that govern the propagation of waves in a solid is the density and the Elastic modulus $[18,19]$. The acoustic impedance $Z$ of the medium can be defined as the resistance of the material to transmit acoustic waves and is determined by 


$$
Z=\rho v
$$

where $\rho$ is the material density and $v$ is the speed of sound in the medium [19]. When the propagating wave reaches an interface (as shown in Figure 1.5), energy is transmitted and reflected. The energy reflected is defined as

$$
R=\frac{Z_{2}-Z_{1}}{Z_{2}+Z_{1}}
$$

where $Z_{1}$ is the acoustic impedance of medium one and $Z_{2}$ is the acoustic impedance of medium two. Figure 1.5 illustrates these transmitted and reflected longitudinal waves for three different media.
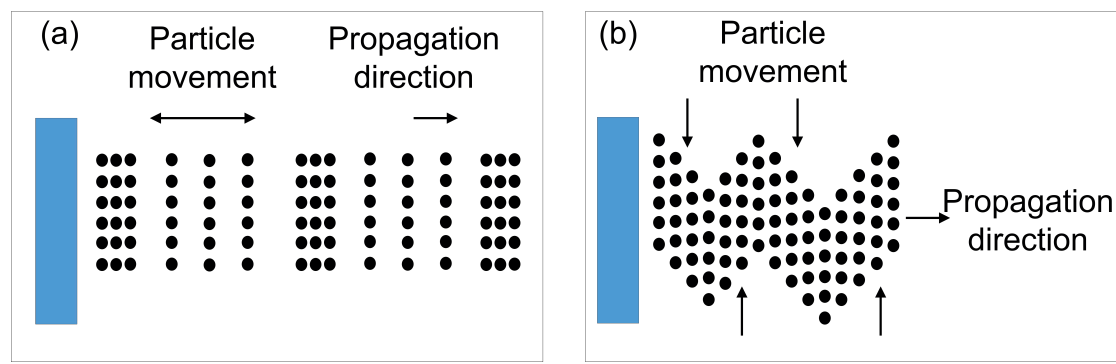

Figure 1.4

(a) Shear wave propagation mode. The movement of the particles is perpendicular to the propagation direction. (b) Pressure wave propagation mode. The movement of the particles is parallel to the propagation direction

Internal defects in structures can be detected from reflections that are recorded between the front interface (Medium 1/Medium 2 in Figure 1.5) and the back interface (Medium 3/Medium 1 in Figure 1.5). Such defects can be porosity, delamination and cracks.

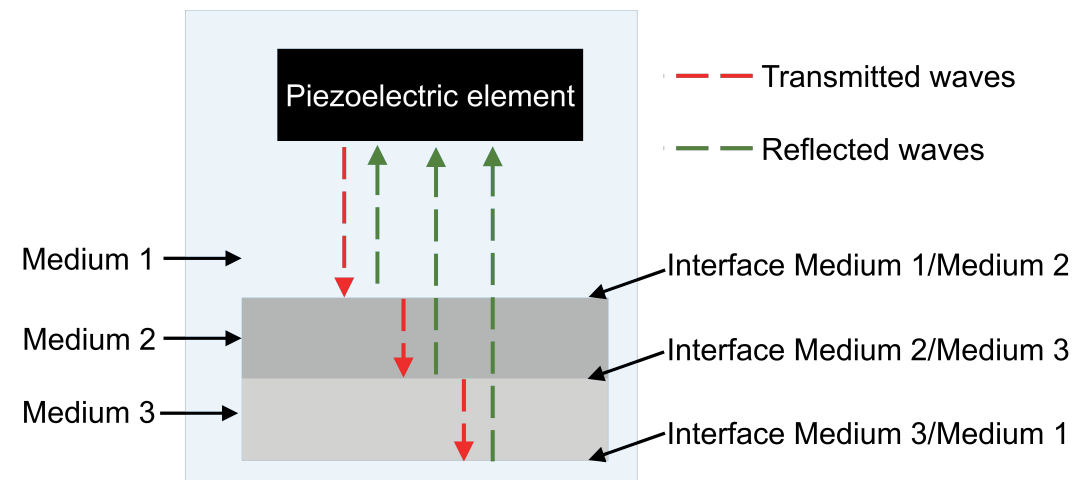

\section{Figure 1.5}

Transmission and reflection of acoustic waves at different interfaces propagating in three different media. 
When the transmitted waves in medium 1 do not travel perpendicularly to the surface of medium 2 (see Figure 1.6), refraction occurs at the interface between the media. Refraction of propagating waves is determined by Snell's law

$$
\frac{\sin \theta_{1}}{v_{1}}=\frac{\sin \theta_{2}}{v_{2}}
$$

where $\theta_{1}$ is the angle of the wave propagating in medium 1 with respect to a vertical line, $\theta_{2}$ the angle of the wave propagating in medium 2 with respect to a vertical line, $v_{1}$ speed of sound in medium 1 and $v_{2}$ is the speed of sound in medium 2. As the angle of the incidence is increased, the angle of refraction increases.

Two critical angles can be found. The first critical angle is when the angle of propagation of the refracted longitudinal is $90^{\circ}$ with respect to the vertical line. This means that the longitudinal wave will propagate along the surface of the specimen. The second critical angle is when the refracted shear wave propagates at $90^{\circ}$ with respect to the vertical line. Thus, the shear wave propagates along the surface as well.

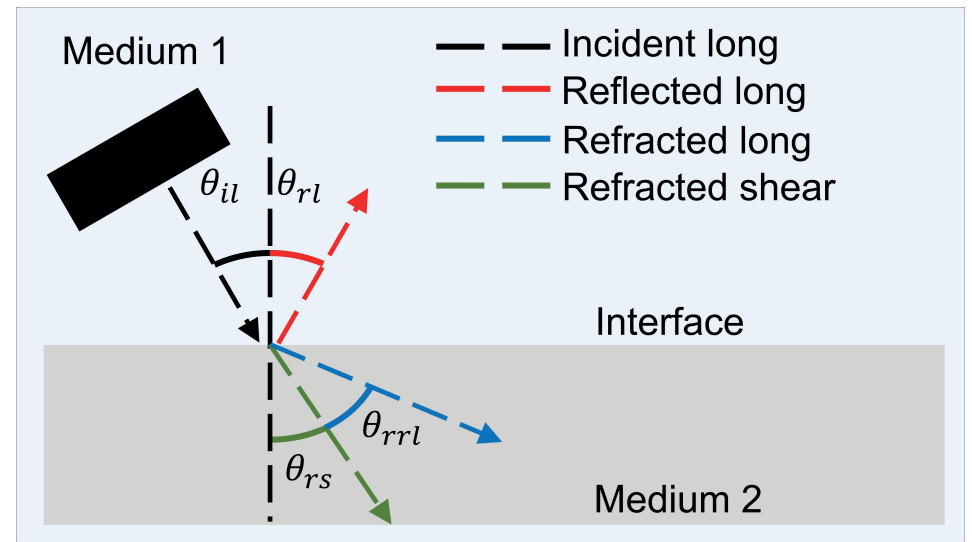

Figure 1.6

Snell's law showing four different waves propagating at four different angles: Incident $\left(\theta_{i l}\right)$, reflected $\left(\theta_{r l}\right)$, refracted longitudinal $\left(\theta_{r r l}\right)$, refracted shear $\left(\theta_{r s}\right)$.

\subsubsection{Ultrasonic testing in drinking water pipes}

In a study performed by Demčenko et al. [20], acidic deterioration in a cementbased specimen was detected with the ultrasonic pulse-echo method. This technique is based on the acoustic impedance difference at the healthy-and degraded interface. A typical cement degraded by acid is shown in Figure 1.7a and its respective ultrasonic signature is depicted in Figure 1.7b.

The main degradation mechanism listed in the literature in cement-based pipes is calcium leaching [21-23]. Naffa et al. [24] showed that the speed of acoustic 
waves in a cementitious structure is a function of its Calcium content. A typical cement degraded by calcium leaching is shown in Figure $1.7 \mathrm{c}$ and its ultrasonic signature is depicted in Figure 1.7d.

Using ultrasound, it is possible to detect degradation for both degradation mechanisms. The difference between these two is that for the former there is a reflection from the degraded layer (see Figure 1.7b), while for the latter there is no reflection from the degraded layer. The reflection from the degraded layer is due to the sharp transition between degraded cement and undamaged cement leading to higher reflection energy, contrary to the case of calcium leaching.

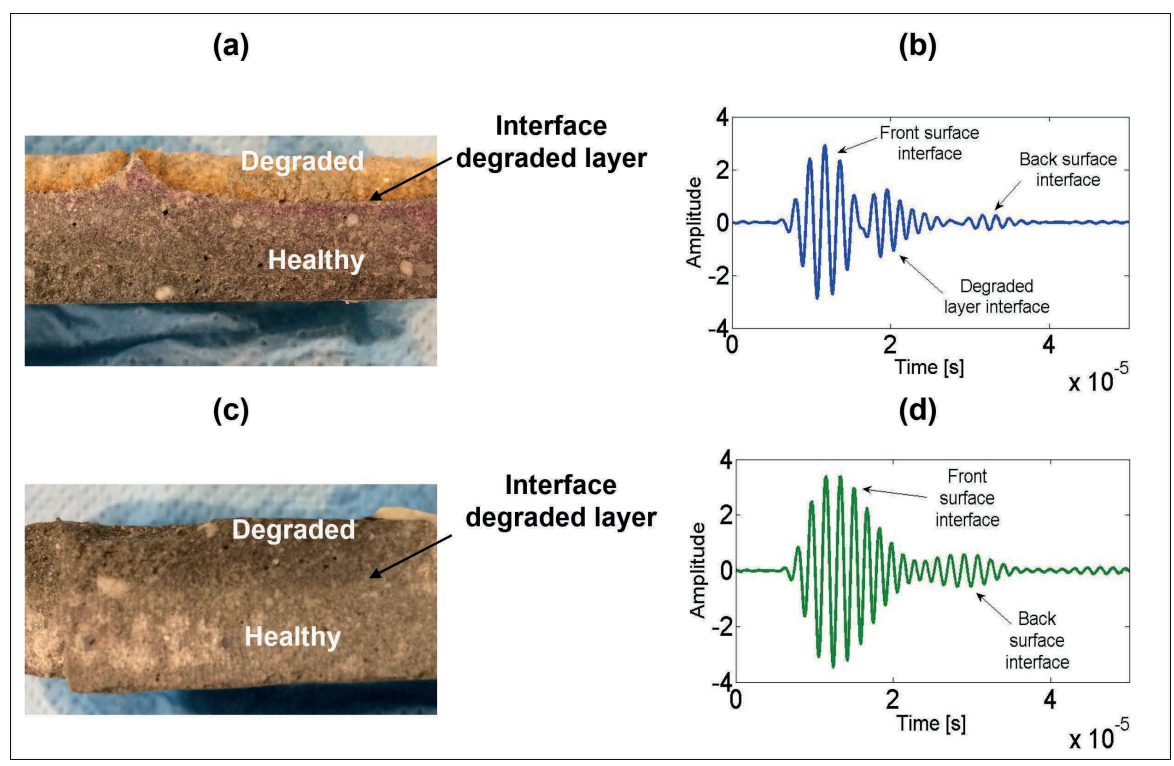

Figure 1.7

(a) Cement degraded by acid and (b) the respective recorded ultrasonic response.

(c) Cement degraded by calcium leaching and (d) the respective recorded ultrasonic response.

Linear ultrasonics have proved to be insensitive to ageing in PVC. This is because linear ultrasonics are sensitive to the changes in the linear elastic regime only. Physical ageing is a reversible process in which the molecule chains of an amorphous polymer strive towards equilibrium [25]. The reason for this is that when the polymer is cooled down from its glass transition temperature (a normal procedure to produce a solid structure), a high thermodynamic non-equilibrium state (in the specific volume and enthalpy) is reached [25]. The physical ageing of PVC has a direct effect on the viscoelastic behaviour (a mechanical property). Viscoelasticity is defined as the time-dependent response of a material under a constant applied stress. This behaviour is best characterized by the creep curves [25]. The effect of aging is better characterized by the shift in time of the creep curves. Creep is defined as the strain change as a function of time at a constant applied stress. The linear regime of the viscoelastic behaviour is small (for some 
polymers), thus it is mostly characterized in the non-linear region. Hutchinson [25] explained the direct correlation between ageing and non-linear creep behaviour.

Demčenko et al. [26] demonstrated that non-linear ultrasound is sensitive to ageing in PVC. They showed that the natural ageing in PVC can be monitored by measuring the amplitude of an acoustic wave generated from mixing two incident acoustic waves inside the PVC material. The latter is known as the wave mixing technique. This wave mixing technique is relatively new and seems very promising for implementation as an inspection technique. A typical laboratory set-up, as used by Demčenko, is shown in Figure 1.8. This two-sided wave mixing configuration is an impractical approach to the inspection of water mains as it requires access to both the inside and the outside surfaces of the pipes. Thus, a one-sided configuration is better suited for pipe inspection.

The way degradation appears in cement-based pipes and in PVC pipes is different: degradation levels in cement-based pipes are revealed when the pipe is cut in a cross-section. Degradation of PVC pipes is not directly visible and there is no sharp change in the aging levels.

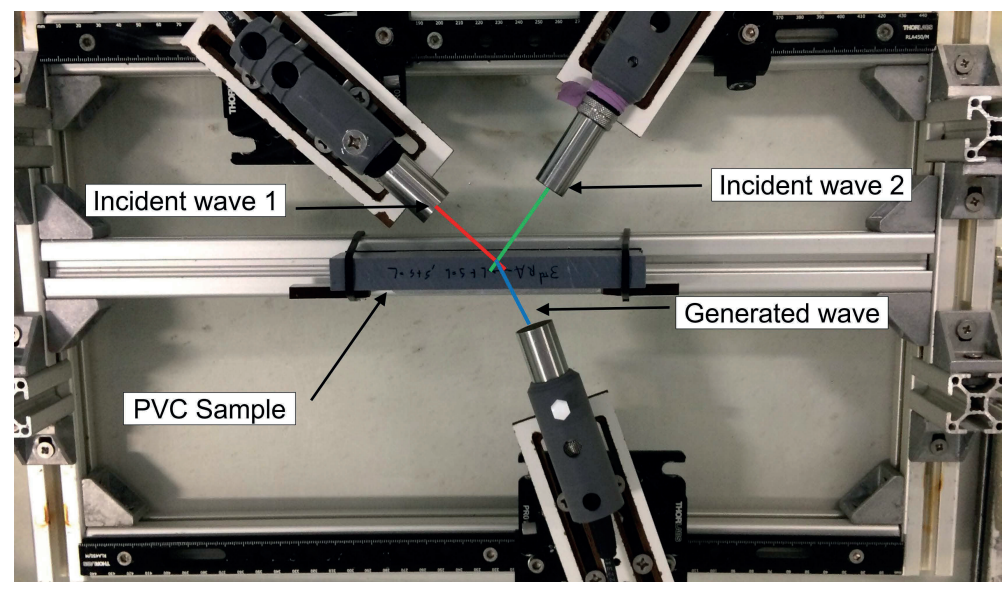

Figure 1.8:

Typical two-sided wave mixing configuration. The red line represents incident wave 1 , the green line represents incident wave 2 and the blue line represents the generated wave.

\subsection{Research aim}

The use of ultrasonic sensors for the inspection of drinking water pipes looks promising. The pulse-echo is a very versatile technique, making it a good candidate for the inspection of AC pipes. For AC pipes, the focus on translating the ultrasonic measurements into an estimate of degradation level. For the PVC pipes, due to the complexity of the wave mixing technique, the focus of this research is on bridging the gap between laboratory environment and field application. The following objectives are set for AC and PVC materials: 
1. Development of an ultrasonic testing method which is capable of detecting and measuring the degradation of a water main section.

2. Upscaling the ultrasonic method from laboratory scale to inline inspection of water main sections.

These objectives can be achieved only if the following questions are answered:

1. How can the degradation state of water mains be determined from ultrasonic measurement data?

a. What is the relationship between measurement results and the test settings?

b. How can a measure for the level of degradation be obtained from the ultrasonic signal? (only for AC)

2. What are the relevant factors to consider for the transition from laboratory conditions to field conditions?

a. How to move from a two-sided to a one-sided approach? (only for PVC)

b. How can a variation in degradation in the circumferential and in the longitudinal direction be determined? (only for AC)

\subsection{Thesis outline}

The thesis is composed of six chapters. In Chapters 2 to 5 , the technological research and development is described in detail. The knowledge gained is integrated and discussed in Chapters 6 and 7 and conclusions are drawn together with some recommendations. In Chapter 2 the degradation mechanisms present in the AC pipes are described in detail as well as the effects of these mechanisms on ultrasonic waves. In that chapter, a methodology to quantify the chemical deterioration is developed and validated. In Chapter 3, the state-of-the-art technology to inspect cement-based pipes is briefly described. Thereafter, the methodology developed in Chapter 2 is tested in an-in service AC pipe. Additionally, the processing algorithms to handle large data sets and extract the relevant parameters are verified.

In Chapter 4, the wave mixing technique is described in detail and a novel methodology for selecting the optimal configuration is developed. The novel approach for the wave mixing technique reduces the complexity of a testing setup. The state-of-the-art inspection technology for PVC is summarized in Chapter 5. Thereafter, the power density of the generated wave is analysed in three different schemes: laboratory tests, field pipe tests and one-sided and two-sided wave mixing configurations. In Chapter 6 the knowledge gained in the previous chapters is integrated into two generalized flow charts showing the major steps and variables to consider for developing inspection techniques (not limited to the drinking water sector). Additionally, in this chapter the status of the inspection methodologies at the end of this $\mathrm{PhD}$ is described. Finally, some ideas on how the drinking water network should be monitored are discussed. In Chapter 7, the conclusion of this work and some recommendations are provided. 


\subsection{Publications}

The work presented in this thesis has resulted in a number of scientific publications and other output.

Published Journal Papers:

- Delgadillo, H. H., Kern, B., Loendersloot, R., Yntema, D., \& Akkerman, R. (2018). A Methodology Based on Pulse-Velocity Measurements to Quantify the Chemical Degradation Levels in Thin Mortar Specimens. Journal of Nondestructive Evaluation, 37(4), 79. (Chapter 2)

- Delgadillo, H. H., Loendersloot, R., Yntema, D., Tinga, T., \& Akkerman, R. (2019). Steering the propagation direction of a non-linear acoustic wave in a solid material. Ultrasonics, 98, pp. 28-34. (Chapter 4)

Submitted Journal Papers:

- Delgadillo, H. H., Geelen, C., Kakes, R., Loendersloot, R., Yntema, D., Tinga, T., \& Akkerman, R. (2019). Ultrasonic inline inspection of a cement-based drinking water main section. Engineering Structures. (Chapter 3)

Ready for Submission to Scientific Journal:

- Delgadillo, H. H., Chidhambaram, N., Loendersloot, R., Yntema, D., Tinga, T., \& Akkerman, R. (2019). Power spectral analysis of a one-sided non-collinear wave mixing test in a PVC pipe. (Chapter 5)

Conference Contributions:

- Delgadillo, H. H., Loendersloot, R., Akkerman, R., \& Yntema, D. (2016, September). Development of an inline water mains inspection technology. In 2016 IEEE International Ultrasonics Symposium (IUS) (pp. 1-4). IEEE.

- Delgadillo, H. H., Loendersloot, R., Yntema, D., Tinga, T., \& Akkerman, R. (2018, October). Experimental Validation of Non-Collinear Wave Mixing Model in a PVC Specimen. In 2018 IEEE International Ultrasonics Symposium (IUS) (pp. 1-9). IEEE.

Submitted Patent:

- H. Hernandez, R.R. Dijkstra, S.v.d. Heide, D. Yntema, R. Loendersloot. Method for measuring cement elements, such as piping, and measurement system there for. Wetsus European Centre of Excellence for Sustainable Water Technology. (2018). 


\section{REFERENCES}

1. E. L. Hall and A. M. Dietrich, 'A Brief History of Drinking Water,' Opflow, vol. 26, no. 6, pp. 46-49, 2018.

2. 'Ancient Engineers\& Inventions,' Anc. Eng. Invent., 2009.

3. E. Paparazzo, 'Surface and interface analysis of a Roman lead pipe 'fistula': microchemistry of the soldering at the join, as seen by scanning Auger microscopy and X-ray photoelectron spectroscopy,' Appl. Surf. Sci., vol. 74, no. 1, pp. 61-72, 1994.

4. G. Balzer and C. Schorn, 'Asset management for infrastructure systems: Energy and water,' Asset Manag. Infrastruct. Syst. Energy Water, pp. 1-339, 2015.

5. H. Whitehouse, 'Hundreds of hospital patients evacuated after burst water pipe floods corridors and even roads outside,' Mirror, 2015. [Online]. Available: https://www.mirror.co.uk/news/world-news/hundreds-hospitalpatients-evacuated-after-6405049.

6. J. Pieters, 'Insurer pays out $€ 34.8$ million to VU Hospital for water damage caused by water main break,' NLTimes, 2016. [Online]. Available: https://nltimes.nl/2016/09/08/insurer-pays-eu348-million-vu-hospital-waterdamage-caused-water-main-break.

7. J. Pieters, 'Water main break floods Den Haag shops,' NLTimes, 2016. [Online]. Available: https://nltimes.nl/2016/05/05/water-main-break-floodsden-haag-shops.

8. 'Hundreds of patients evacuated as Amsterdam hospital floods,' DutchNews, 2015. [Online]. Available: https://www.dutchnews.nl/news/2015/09/some500-patients-evacuated-as-amsterdam-hospital-floods/.

9. R. H. S. Beuken, H. De Kater, and J. H. G. Vreeburg, 'Securing Water and Wastewater Systems,' Secur. Water Wastewater Syst., pp. 149-159, 2013.

10. P. J. J. . Geudens and J. Grootveld, 'Dutch Drinking Water Statistics 2017,' 2017.

11. 'Infosheet Smart water Grids Thema Wetsus,' 2018.

12. H. A. Visser, 'Residual lifetime assessment of uPVC gas pipes,' University of Twente, Enschede, The Netherlands, 2010.

13. E. Drenth, 'Residual lifetime of uPVC pipes,' University of Twente, Enschede, The Netherlands, 2015.

14. A.M. Arsénio, Lifetime prediction of PVC push-fit joints. 2013.

15. A. Demcenko, 'Development and analysis of noncollinear wave mixing techniques for material properties evaluation using immersion ultrasonics,' Cambridge University Press, Enschede, The Netherlands, 2014.

16. P. Van Thienen, M. Maks, D. Yntema, and J. Janssens, 'Continuous robotic inspection of pipes for data rich asset management,' no. September, pp. 1-7, 2017.

17. A. Arnau and D. Soares, 'Fundamentals of Piezoelectricity,' in Piezoelectric Transducers and Applications, Berlin, Heidelberg: Springer Berlin Heidelberg, 2008, pp. 1-38.

18. K. F. Graff, 'Wave motion in elastic solids.' Dover Publications, New York, 1975. 
19. 'NDT Resource Center.' [Online]. Available: https://www.ndeed.org/EducationResources/educationresource.htm.

20. A. Demčenko, H. A. Visser, and R. Akkerman, 'Ultrasonic measurements of undamaged concrete layer thickness in a deteriorated concrete structure,' NDT E Int., vol. 77, pp. 63-72, 2016.

21. D. Wang and D. R. Cullimore, 'Bacteriological challenges to asbestos cement water distribution pipelines,' J. Environ. Sci., vol. 22, no. 8, pp. 1203-1208, 2010.

22. D. Wang, Y. Hu, and R. Chowdhury, 'Examination of Asbestos Cement Pipe Deterioration with Scanning Electron Microscopy,' in Pipelines 2011, 2011, no. 306, pp. 65-78.

23. R. Chowdhury, Y. Hu, and D. Wang, 'Condition Evaluation of Asbestos Cement Water Mains,' in Pipelines 2012, 2012, no. 306, pp. 288-297.

24. S. Ould Naffa, M. Goueygou, B. Piwakowski, and F. Buyle-Bodin, 'Detection of chemical damage in concrete using ultrasound.,' Ultrasonics, vol. 40, no. 1-8, pp. 247-51, 2002.

25. J. M. Hutchinson, 'Physical aging of polymers,' Prog. Polym. Sci., vol. 20, no. 4, pp. 703-760, Jan. 1995.

26. A. Demčenko, R. Akkerman, P. B. Nagy, and R. Loendersloot, 'Non-collinear wave mixing for non-linear ultrasonic detection of physical ageing in PVC,' NDT E Int., vol. 49, pp. 34-39, 2012. 



\title{
Chapter

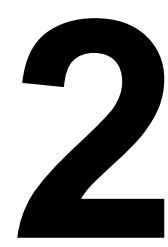

\section{A methodology based on pulse-velocity measurements to quantify the chemical degradation levels in thin mortar specimens}

\begin{abstract}
Ultrasonic pulse-echo measurements are used to quantify through thickness chemical degradation in thin mortar specimens. The degradation levels are predicted from the time of travel of the acoustic wave through the thickness of the structure. The front and back wall reflections are used to obtain additional information from very early stage degradation. The pulse-velocity of acoustic waves as a function of the thickness of the layers within the structure is described. Based on the pristine and fully degraded conditions of the mortar, the complete range of degradation levels over the specimen's thickness can be estimated. The method is applicable for leaching of calcium and acidic attack. The acoustic measurements were verified with destructive testing. The correlation between the acoustic and non-acoustic tests agree with the described pulse-velocity and degraded depth function. This methodology can be used for any type of thin-layered structures.
\end{abstract}

This chapter is based on: H. Hernandez Delgadillo, B. Kern, R. Loendersloot, D. Yntema, and R. Akkerman, "A Methodology Based on Pulse-Velocity Measurements to Quantify the Chemical Degradation Levels in Thin Mortar Specimens," J. Nondestruct. Eval., vol. 37, no. 4, pp. 1-13, 2018. 


\subsection{Introduction}

The installation of a large part of the drinking water network in many countries was done in the mid-twentieth century. Nearly $30 \%$ of the Dutch water mains network is made of cementitious materials. Depletion of material initiated after the pipes were laid underground, so up until now, these assets have been under continuous deterioration. Deterioration leads to a decrease in load capacity and higher chance of malfunction. An inspection system capable of detecting and quantifying the amount of degradation within cement-based drinking water pipes will enhance the maintenance of the infrastructure and potentially avoid pipe failure.

The degradation mechanisms inherent in cementitious drinking water pipes are: leaching of calcium, acidic attack, carbonation, biodegradation and sulphate attack [1]. Deterioration of these cementitious pipes primarily occurs due to leaching of calcium. On the contrary, sulphate attack and acidic attack are less likely to occur. Yet, it has been shown that the generation of a layer of bacteria (biofouling) in the inner surface of the water distribution pipes will increase the acidity level $[2,3]$. Deterioration in the outer surface of the pipe can occur due to mixed degradation mechanisms [1]. Furthermore, internal porosity in the structure leads to more complex interaction between inner and outer degraded layers [1].

Carbonation of cement is mainly due to the percolation of carbon dioxide $\left(\mathrm{CO}_{2}\right)$. Such condition in the drinking water supply system can occur if the water is saturated with $\mathrm{CO}_{3}^{2-}$ and it is commonly found in the outer surface of the drinking pipes [1-3]. In the carbonation process, $\mathrm{CO}_{2}$ penetrates through the porous solid material and react with the cement phases by leaching the calcium. Furthermore, subsequent reactions will enhance the mechanical properties of the structure [2, 4]. This will only occur in the internal surface if there is oversaturation of $\mathrm{CO}_{3}^{2-}$ and if $\mathrm{CO}_{2}$ is trapped in the soil in the vicinity of the outer surface of the pipe.

A cementitious structure in long-term contact with water will leach out the free lime and the calcium content from the different phases of the cement. Specifically, the contact with low $\mathrm{pH}$ and low ion content water. This mechanism has been extensively reported and it decreases the strength of the structure. Largely because it is driven by diffusion of calcium towards the aggressive medium [5-7].

\subsubsection{Condition assessment methods}

There are three methods currently available for inspection of cement-based pipes: acoustic, ground penetrating radar (GPR) and the phenolphthalein test. The acoustic method consists on monitoring the speed of sound waves traveling in longitudinal wave mode from two fixed points in the pipe section, providing information about the average state of the material between the measurement points. The GPR method consists in transmitting radio waves into the outer surface of the pipe. The waves are reflected/transmitted depending on the changes in the material properties and it is used mostly for inspection of sewage pipes. For a correct inspection of the structure, the pipes have to be dug out, which 
is costly and time-consuming. Similarly, the phenolphthalein test requires the pipes to be dug out and it is a destructive testing. Having possible hazardous components inside makes this test even more complicated.

The objective of this research is to detect anything from early degraded levels to full degradation in a mortar specimen from two chemical deterioration mechanisms: calcium leaching and acidic attack. Secondly, a methodology is presented which determines the amount of degradation by pulse-velocity measurements through the thickness of the thin layered mortar. The measurements were performed on mortar specimens under laboratory conditions; no drinking water pipes were studied. Yet, this research is driven by stepping forward to the development of a non-destructive evaluation methodology that can potentially be implemented in cementitious (without ferrous components) drinking water pipes.

\subsubsection{Ultrasonic testing in cement-based structures}

The material state of the water mains can be detected by means of ultrasonic nondestructive evaluation (NDE). The long-term interaction of the pipes with the conveyed water and surrounding soil induces deterioration from both the internal and external surfaces of cementitious pipes. The acoustic signature of these layers can be found with the ultrasonic pulse-echo (UPE) technique. Demčenko et al. [8] demonstrated that the UPE is able to detect degradation due to acidic attack, based on finding a reflection from the healthy-degraded interface. The main drawback of this technique is that it cannot detect degradation levels that are smaller than the measurement wave length. Increasing the measurement frequency $(1 \mathrm{MHz})$ improves the resolution near interfaces, but at the cost of higher signal attenuation. On the contrary, lower measurement frequencies (approximately $500 \mathrm{kHz}$ ) will compromise near surface resolution but will increase the signal-to-noise ratio from the back-wall reflection. A trade-off between the signal strength and the near-surface resolution has to be found. Furthermore, detection of chemical deterioration has been demonstrated by monitoring the change in speed of sound $[9,10]$.

Low frequency ultrasonic testing $(50 \mathrm{kHz}-500 \mathrm{kHz})$ has been extensively performed in cement-based structures [10-15]. Some of these techniques are pulse-echo and ultrasonic wave diffusion. Diffusion of ultrasonic waves has been used to measure crack depth in concrete with a range of 400 to $600 \mathrm{kHz}$ [11]. This technique has been used likewise to characterize the dissipation and diffusion coefficients from ultrasonic waves in concrete structures [12] with the highest frequency at $800 \mathrm{kHz}$. Furthermore, mechanical properties from early hydration setting of cement have been characterized by pulse velocity measurements [13]. Compressive damage and porosity estimation were studied from measuring speed of sound in the concrete as well [10,14-15]. The majority of these studies have been carried out with frequencies bellow $800 \mathrm{kHz}$ due to the high scattering and attenuation of the acoustic waves in cement-based structures. 
One study showed the possibility to characterize the properties of cement during production based on attenuation and pulse velocity measurements [16]. Damage in cementitious structures such as cracks and inclusions were investigated as well with the wave guide technique [17, 18]. Alternatively, the properties of cement during production were also studied with the guided wave technique [19].

\subsubsection{Cement hydration and main phases}

The formation of cementitious structures is mainly due to the hydration of cement. Production of Portland cement typically comes from the decomposition of limestone (calcium carbonate) and the addition of $\mathrm{m}$-kaolin in a process called clinkering which then generates new compounds [20]. The main phases present in Portland cement after clinkering are: tricalcium silicate or Alite (3CaO.SiO2 or C3S), dicalcium silicate or Belite (2CaO.SiO2 or $\mathrm{C} 2 \mathrm{~S})$, calcium alumino ferrite (4CaO.Al2O3Fe2O3 or $\mathrm{C} 4 \mathrm{AF}$ ) and tricalcium aluminate $\mathrm{C} 3 \mathrm{~A}$-alkali solid solution (3CaO.Al2O3 or C3A). The content of each phase in the total mixture can be controlled by the processing conditions as well as the mole fraction of $\mathrm{m}$-kaolin to calcium oxide. In practice, the phases that provide higher strength and durability to the cementitious structures are approximately $45 \%$ and $25 \%$ of Alite and Belite respectively [20]. The resultant components of hydrating the main phases are the calcium silicate hydrates, better known as CSH gels, and the Portlandite or better known as calcium hydroxide. These two components contribute to the major part of the structural capacity. The hydration of the other phases will form lower amount of $\mathrm{CSH}$ and Portlandite but mainly will form Ettringite (depending on the Gypsum content) and mono-sulphoaluminate hydrates [20, 21].

\subsubsection{Acidic deterioration}

An acidic environment can be very harmful for cementitious structures. Moreover, the acid dissolves and crumbles completely the binder material leaving no structural strength. Wang et al. [1] reported that it is highly unlikely to find a low $\mathrm{pH}$ in the drinking water thus no contact with an acidic environment would be expected. Nonetheless, the generation of bacteria layer (biofouling) in the internal surface is of acidic nature. It has been shown that this layer will attack the structure in an acidic manner [1-4]. The contact of cement with acid dissolves the Calcium within the structure. Thus, the main hydrate compounds (calcium hydroxide and calcium silicate hydrates) are leached and dissolved. The time at which these reactions occur is dependent on the acidity of the solution. The rest of the hydrated products are decomposed [21-23] leading to material crumble. Figure 2.1 depicts a cross-section of a cementitious structure subjected to one-sided (upper surface) acidic deterioration.

The change in the structure can be clearly seen between degraded and nondegraded material (see Figure 2.1). Further, in the degraded zone, a thin brownish coloured layer is present at the end of the degraded zone. This ferric hydroxide layer accumulates the salts that are being removed from the binder. The later was 
reported by Chandra [21] where she concluded that just after the brown ring, the calcium content completely drops due to dissolution. The ferric hydroxide layer remains with a constant thickness. In the degraded zone there is no binder anymore and the remaining compounds and aggregates can be effortlessly removed.

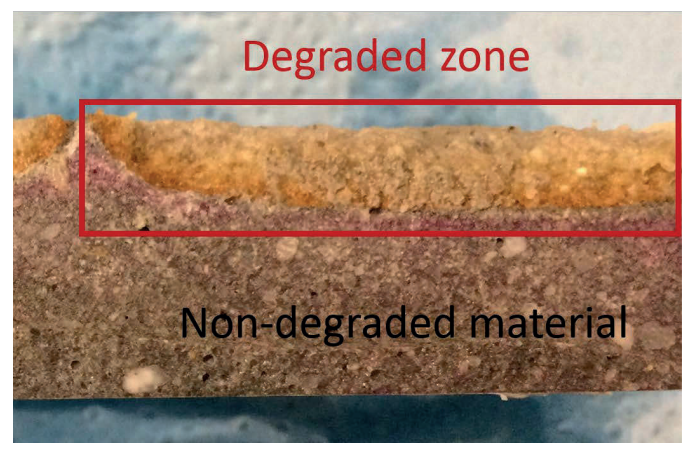

Figure 2.1

Acidic deterioration in cementitious material. Cross-section of specimen subjected to degradation from only one side. Sharp transition between pristine and degraded material.

\subsubsection{Calcium leaching}

Dissolution of Portlandite is the dominant reaction during contact with low ion content water [24]. Immediately after the Portlandite has been leached, the compounds that react are the monosulfoaluminate, ettringite and the progressive dissolution of the CSH gels [21-23]. Leaching of calcium does not dramatically reduce the structure's strength as compared to acidic deterioration.

The dissolution of Portlandite begins when the material is placed in contact with low-ion content water. The $\mathrm{pH}$ of the solution is dependent on the minerals present in the water. For instance, studies have shown that the measured $\mathrm{pH}$ from a drinking water supply system is 6.9 [24]. In another study, the recommended $\mathrm{pH}$ level in the drinking water is $8.2 \pm 0.1$ [25]. However, in a drinking water supply system, the measured $\mathrm{pH}$ is directly dependent on the location.

From the transport law of diffusion and dissolution, the mass balance equations are

$$
\frac{\partial\left(\varnothing \cdot C_{C a}\right)}{\partial t}+\frac{\partial S_{C a}}{\partial t}=\nabla \cdot\left(D \cdot \nabla C_{C a}\right)
$$

where $C_{C a}$ is the calcium concentration in the pore solution, $S_{C a}$ is the real mass density of the solute, $D$ is the diffusivity of calcium in the pore solution, $\varnothing$ is a factor related to the porosity in the solid, $\nabla C_{C a}$ is the concentration gradient [29$31]$. This is a complex phenomenon that has been extensively studied [26, 27]. 
Figure 2.2 depicts the degradation profile of a specimen's cross-section. The part in the red box shows the material that was subjected to leaching. Leaching in this case originated from the top surface. There is no clear sharp transition nor visual difference in the material structure. The degraded material in this case has much higher remaining strength capacity compared to a material deteriorated by acid (Figure 2.1).

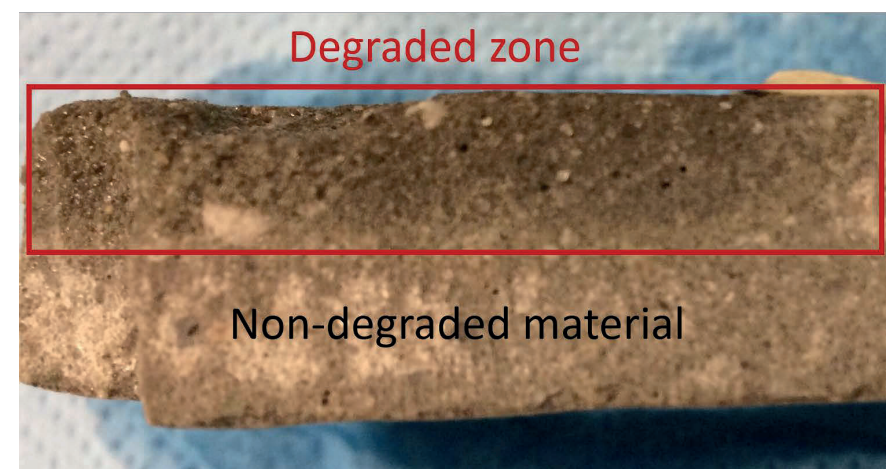

Figure 2.2

Leaching of calcium in cementitious material. Transition between pristine and degraded material is composed of gradual layers of calcium content.

\subsubsection{Effect of calcium content on the speed of sound}

The decrease in Calcium content as shown in Equation 2.1 has a direct effect in the mass of the solid and thus a direct effect on the density $\rho$. Local densities will change as degradation penetrates the solid which in turn changes the acoustic velocity. The speed of sound in a solid medium is defined as

$$
V=\left(\frac{E(1-v)}{\rho(1+v)(1-2 v)}\right)^{1 / 2}
$$

where $E$ is the Young's modulus and $v$ is the Poisson's ratio.

\subsubsection{Layers present in degraded cement}

If the cementitious structure has very low permeability or if the properties of the cementitious structure are leaching resistant, two layers can be found in the degraded zone [26-28, 30, 31]: A fully leached layer and a partially degraded layer with a gradient of Calcium content (see Figure 2.3a). The intermediate layer has a negligible thickness compared to the pristine and fully degraded thicknesses.

The latter has only decalcification of the calcium silicate hydrates. On the contrary, if no admixtures are included during the setting of the cement or the cement has high permeability, the leached layer is composed of three internal layers (see 
Figure 2.3a). The two intermediate layers have a comparable thickness with respect to the pristine and fully degraded thicknesses [26-28, 30, 31].
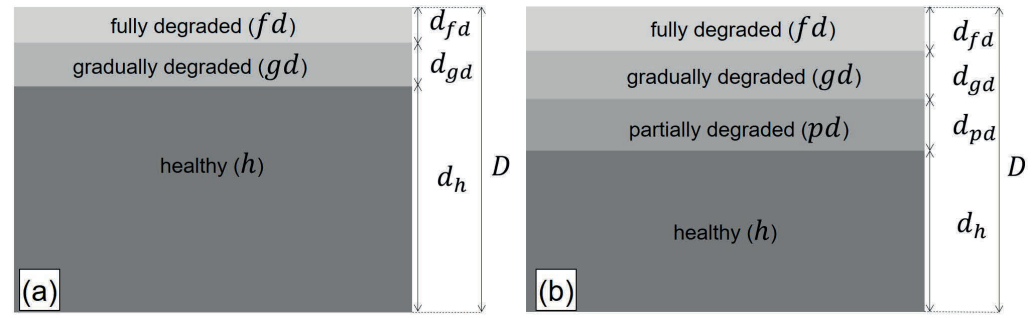

Figure 2.3

Leached cementitious structure a) with low permeability and degradation resistant and b) with high permeability and non-degradation resistant admixtures

The gradually degraded layer has a gradient of calcium content from the calcium hydroxide dissolution and decalcification of the $\mathrm{CSH}$. The partially degraded layer has only dissolution of calcium hydroxide. The density has a direct effect due to the calcium content in the different layers and thus the acoustic properties as well. The layered structures defined in Figure 2.3 are a function of the calcium content. Figure 2.3a and Figure 2.3b are two different cases of calcium leaching. For acidic attack only Figure 2.3a represents the calcium content distribution. Experimental evidence of the calcium distribution along the thickness, the mechanical strength and the porosity can be found in the literature [20-23, 26-31].

\subsection{Methods}

In order to characterize the response of the ultrasonic waves to the long-term degradation of cement-based pipes in a laboratory scale, the following steps are proposed: 1) production of mortar specimens and preparation; 2) accelerated degradation of specimens; 3) ultrasonic test; 4) signal processing; 5) estimation of degraded depth; and 6) destructive testing. As mentioned earlier, degradation in cementitious drinking water pipes takes place from the inner and outer surface. The exposure time intervals are explained in the following section. It is important to note that the same procedure was followed for each degradation mechanism separately. Thus, the specimens submerged in one solution were not submerged in the other solution. In this way there was no interaction between the two degradation mechanisms. Finally, the effect of the material composition on the response of the ultrasonic signals to degradation was investigated by manufacturing two different specimen batches with different material composition.

\subsubsection{Production of specimens and preparation}

Mortar blocks with thickness of $20 \mathrm{~mm}$ were manufactured with Portland cement (CEM I). The recipe of the manufactured specimens is shown in Table 2.1. 
Table 2.1 Composition mortar specimens

\begin{tabular}{ccccc}
\hline w/c & CEM I & $\begin{array}{c}\text { Sand } \\
\text { Ratio }\end{array}$ & $\begin{array}{c}\text { Plasticizer } \\
(\mathrm{kg})\end{array}$ & $\begin{array}{c}\text { Water } \\
(\mathrm{kg})\end{array}$ \\
0.3 & 0.827 & 1.274 & 0.021 & 0.247 \\
0.4 & 0.718 & 1.274 & 0.0061 & 0.287 \\
\hline
\end{tabular}

A coating was placed on the back of the specimens and in between the areas to be degraded in order to obtain many degraded states in one specimen and to avoid the double-sided degradation for the one-sided degradation tests. The specimens were kept immersed in water after the curing time, during storage and during the ultrasonic tests. Water saturation was kept during the experiments.

\subsubsection{Accelerated degradation}

The specimens from batch 1 were immersed in 6 molar ammonium nitrate (AN) solution and the specimens from batch 2 were immersed in $1 \%$ concentration hydrochloric acid $(\mathrm{HCL})$ solution. The measured initial $\mathrm{pH}$ values are 5 for the ammonium nitrate solution and 1 for the hydrochloric acid solution. The degradation interval times for the one-sided experiments were divided in early stage with $1,2,3,4,5,6$ and 7 days of exposure to the solution and long deterioration with, 21, 35, 49, 63, 77 and 91 days of exposure to the solution. For the two-sided degradation the interval times were 7,14 and 28 days. The solutions were renewed every two days in order to maintain a constant degradation rate. Acceleration of calcium leaching can be done by ammonium nitrate solution [9]. The reaction continues with the mono-sulfoaluminates, ettringite and finally to the progressive decalcification of the gels. The calcium leaching does not break down the gels but only gradually dissolves the calcium and thus the compound is still present. On the contrary the acid compound completely fragments the $\mathrm{CSH}$ gels.

\subsubsection{Ultrasonic test}

Dispersive behaviour has been reported extensively reported [8, 16-18]. Wave distortion as well as group velocity are the two main challenges found in ultrasonic testing in cement-based structures due to dispersion. Coarse grain structure, porosity and composite nature of the cement-based structures are the main contributors to this phenomenon. In similar studies Aggelis et al. [16] measured the attenuation, frequency response and dispersion of ultrasonic waves as a function of the sand content and water-to-cement ratio of the mortar specimens. He concluded that the main source of error is the detection of the time delay of the signals in the time-domain. The dispersive characteristic is very important to consider; however, dispersion of ultrasonic waves has negligible effect on the measurements performed due to the signal processing scheme presented. Furthermore, the previous work shows that the dispersion of ultrasonic waves at 
the frequencies proposed in this research are minimal in comparison with the change in pulse velocity in a degraded mortar [8].

The specimens were placed in an immersion tank (free of degradation agents) as shown in Figure 2.4. A uniform coupling between the transducer's surface and the specimen was achieved. In an inline inspection, it is possible to access to the internal which is one of the main constraints. Therefore, pulse-echo is the most suitable technique to measure the speed of sound in the structure. The tests were performed on the deteriorated side (for the one-sided degradation). Two flat nonfocused transducers with $0.5 \mathrm{MHz}$ and $1 \mathrm{MHz}$ central frequencies were used with 0.75 " and 0.5 " diameters respectively. The data was recorded with a 16 bits resolution digitizer at a $62.5 \mathrm{MS} / \mathrm{s}$ sample rate.

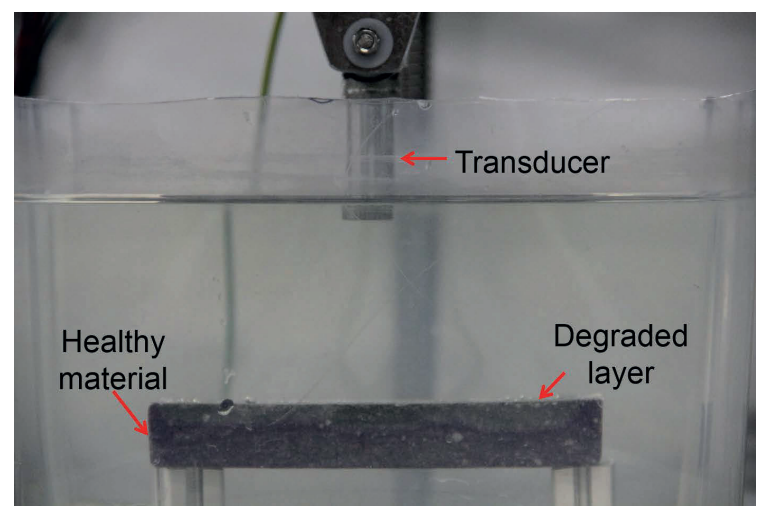

Figure 2.4

Immersion ultrasonic test set up with a degraded cementitious specimen.

(a)

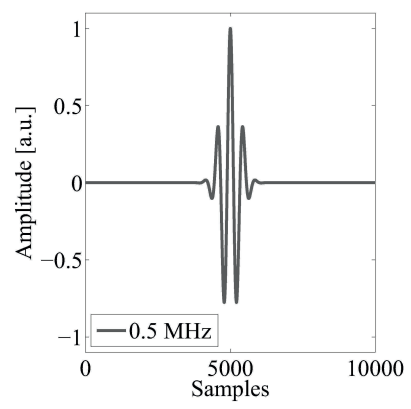

(b)

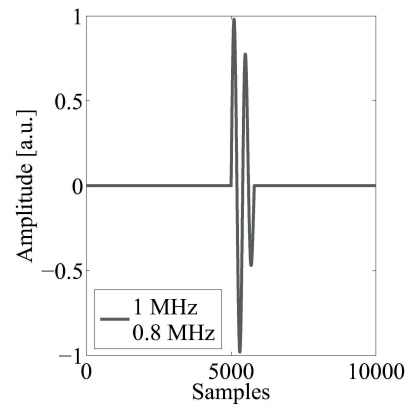

Figure 2.5

Input signals with a) Windowed sine; b) exponential decrease sine.

The input voltage used is shown in Figure 2.5. A windowed sinusoidal signal was applied to the $0.5 \mathrm{MHz}$ transducer and a sinusoidal signal with exponential decrease was applied to the $1 \mathrm{MHz}$ transducer. In the latter two different excitation 
frequencies were used: $0.8 \mathrm{MHz}$ and $1 \mathrm{MHz}$. The response of the transducer to the second input signal further reduced the resonance of the piezoelectric element. The frequency components of such input signals were very important for the signal processing procedure. Each of the input signals has a specific set of frequency components and the ringing of the transducer was reduced allowing spatial resolution of close reflections. On the contrary, spike-shaped excitation signals generate a long resonance response of the piezoelectric element as well as higher energy harmonics [10].

\subsubsection{Signal processing}

The effect of acoustic and electronic noise can be reduced by dedicated hardware components or software implementation. Digitally processing the data requires the analysis and the implementation of processing algorithms. Nevertheless, one of the main advantages of post-processing is that the parameters of interests can be accurately extracted without the need of calibration procedures. In this work, the main parameters to extract are the amplitude and time of arrival from the water-cement interface (front wall) and the cement-water interface (back wall). Thus, following scheme is proposed: 1) noise removal (by Wiener Filtering); 2) analytic envelope calculation.

\subsubsection{Noise removal}

As mentioned, the Wiener filter is used for noise reduction and the removal of frequencies uncorrelated to the ultrasonic waves. This procedure enables to extract the specific frequency components to the signal of interest. The amplitude and time of arrival from the reflected signals are the main parameters for solving this formulation. However, a priori information has to be known from the scattering amplitudes. Thus, the transfer function has a particular value for each reflected wave, in this case, the front wall reflection and the back-wall reflection. The model presented by Neal et al. [32] is

$$
y(t)=h(t) * x(t)+n_{a}(t)+n_{e}(t)
$$

where $y(t)$ is the signal recorded with the acquisition unit, $x(t)$ is the unknown impulse response function of the scattering amplitude, $h(t)$ is the transfer function of the system, $n_{a}(t)$ is the acoustic noise (porosity, impurities, internal interfaces) and $n_{e}(t)$ is the electronic noise. The transfer function $h(t)$ is the system response and it considers the voltage input to the sensor, the response from the electronics, the response from the transducer and the propagation effects of sound waves in the material (diffraction, attenuation, interface of mediums, etc.) [32-35]. The noise components can be grouped in a single term $n(t)$. Equation 2.3 is transformed into the frequency domain

$$
Y(\omega)=H(\omega) X(\omega)+N(\omega)
$$


Solving for $X(\omega)$ directly does not give meaningful results due to the mathematical ill posed problem [32]. $X(\omega)$ is solved from a statistical point of view where $X(\omega)$ and $N(\omega)$ are uncorrelated Gaussian random variables with known variance and mean. Thus, $Y(\omega)$ is a random Gaussian random variable as well. The transfer function $H(\omega)$ is a non-random known value [32]. The estimated value of $X$ can be obtained from the maximum value of the probability density function $f(X / Y)$ if this is a Gaussian random variable. Details of the derivation can be found in literature [32]. The estimated value of $X$ is obtained in the frequency domain

$$
\hat{X}(\omega)=\left[\frac{H^{*}(\omega)}{|H(\omega)|^{2}+\sigma_{N}^{2} / \sigma_{X}^{2}}\right] Y(\omega)
$$

The estimated vale $\hat{X}(\omega)$ is then transformed to the time domain as $\hat{x}(t)$ [32-35].

\subsubsection{Analytic envelope (Hilbert transform)}

Accurate amplitude and time of arrival can be obtained from the extraction of the analytic envelope if noise has been removed [37-40]. In order to extract the analytic envelope, the square root of the sum of the squares of the real and imaginary parts are calculated for each point in time

$$
a(t)=\left(\left(x_{r}(t)\right)^{2}+\left(\frac{1}{\pi} \int_{-\infty}^{\infty} \frac{x_{r}\left(t^{\prime}\right)}{\left(t-t^{\prime}\right)} d t^{\prime}\right)^{2}\right)^{1 / 2}
$$

where $x_{r}$ is the real part of the signal and the second term is the Hilbert transform [37]. Finally, the time of arrival is extracted from the maximum peak from the respective reflections of interests, in this case the front wall and back wall reflections [36-40].

The processing steps for the experiments performed in the mortar under acidic attack are depicted in Figure 2.6. The blue line represents the raw signal, while the red and black lines represent the data after Wiener filter for the front wall reflection and back wall reflection respectively. The dotted line represents the analytical envelope of the filtered data.

As mentioned in the previous section, the change in the material properties is sharp in an acidic deterioration environment. For this reason, it is possible to see a reflection from the deteriorated layer. In the literature, the detection mechanism is based on such reflection [8]. Nevertheless, it was found that such reflection cannot be found in a calcium leaching scenario. Furthermore, Figure 2.7 depicts the typical signal recorded from the experiments of a mortar degraded by contact with ammonium nitrate solution. The red lines and the black lines enclose the part of the signal that is used to obtain the frequency components and perform the Wiener filter. The signal-to-noise ratio clearly improves the detection of the reflection of interest. 


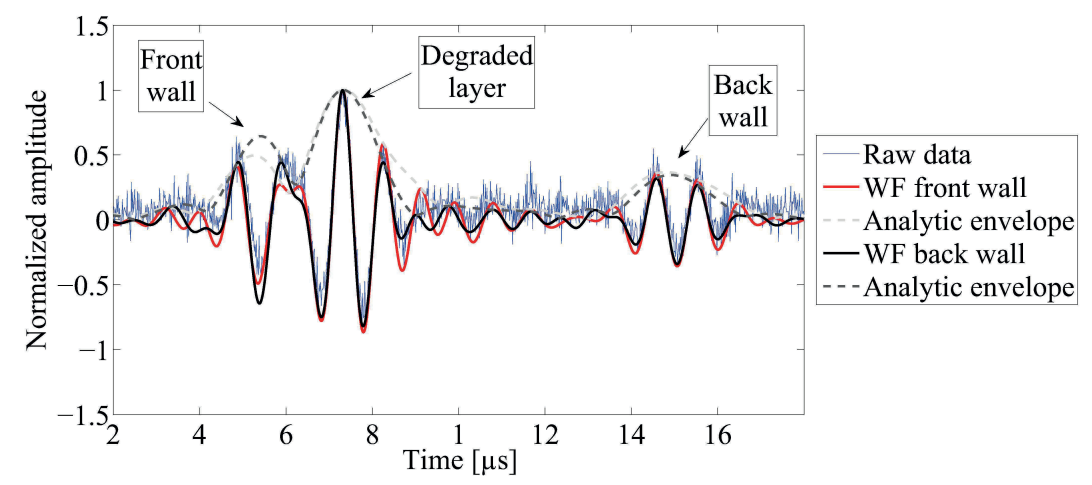

Figure 2.6

Typical data for acidic deterioration obtained after 3 days in one-sided acidic solution. The signal processing steps are shown as the Wiener filter and analytic envelope.

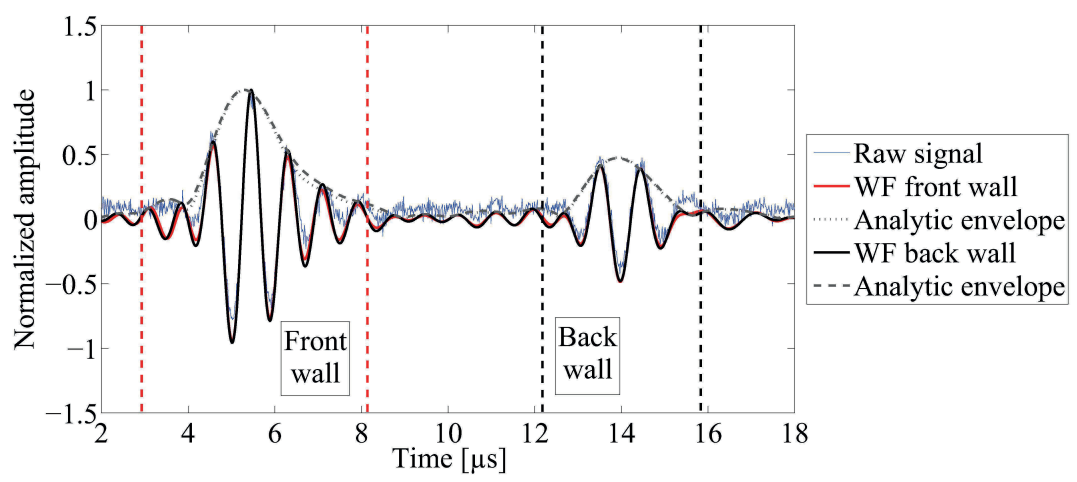

Figure 2.7

Typical data for calcium leaching obtained after 4 days in one-sided deterioration configuration. The signal processing steps are shown as the Wiener filter (WF) and analytic envelope.

\subsubsection{Estimation of degraded depth}

The estimation of degraded depth from measuring the acoustic velocity is directly related to the degraded depth measured from the destructive test. One way to do this is to firstly characterize the speed of sound in pristine and fully degraded material. The total time that the waves take to travel through the structure is

$$
t_{D}=t_{h}+t_{p d}+t_{g d}+t_{f d}
$$


where the subindex $p d$ is partially degraded, $g d$ is gradually degraded and $f d$ is fully degraded. Then the distance over velocity is substituted:

$$
\frac{D}{V_{D}}=\frac{d_{h}}{V_{h}}+\frac{d_{p d}}{V_{p d}}+\frac{d_{g d}}{V_{g d}}+\frac{d_{f d}}{V_{f d}}
$$

the remaining healthy wall thickness is then substituted by

$$
d_{h}=D-d_{p d}-d_{g d}-d_{f d}
$$

The following relations are established in terms of the degraded depth and the speed of sound in healthy material

$d_{p d}=a d_{d} ; \quad d_{g d}=b d_{d} ; \quad d_{f d}=c d_{d} ; \quad V_{p d}=\alpha V_{h} ; \quad V_{g d}=\beta V_{h} ;$

$V_{f d}=\gamma V_{h}$

where, $a, b$ and $c$ are the fraction of material with respect to the total degraded area and $\alpha, \beta$ and $\gamma$ are the fraction of speed of sound with respect to healthy material. Substituting these relations into Equation 2.8 and solving for the degraded depth $d_{d}$, the following is obtained

$$
d_{d}=\frac{D}{V_{D}}\left(\frac{V_{h}-V_{D}}{\frac{a}{\alpha}+\frac{b}{\beta}+\frac{c}{\gamma}-1}\right)
$$

where $V_{D}$ is the speed of the total thickness, $V_{h}$ is the speed of sound in pristine material and $D$ is the thickness of the specimen. From Equation 2.10 the degraded depth can be predicted from the ultrasonic measurements. This relationship was corroborated with destructive testing.

The equations 2.7 to 2.10 describe the speed of sound distribution in a layered medium. The equations do not take in consideration the propagation of sound waves in a solid elastic medium. Thus, in this scheme the dispersion, attenuation and absorption of sound waves are not included in the quantification of degradation. The interaction of reflections as well is not considered in the equations described in this section.

\subsubsection{Destructive testing and validation}

In order to measure the degraded level with non-acoustic method, the specimens were cut in the middle of the degraded areas. For the calcium leaching case, the degraded depth cannot be seen directly. A Phenolphthalein solution was sprayed in the fresh cut surfaces. If the $\mathrm{pH}$ of the surface is higher than 8.2 then the solution remains purple, this is an indication of non-degraded material. On the contrary if the $\mathrm{pH}$ of the specimen's surface is below 8.2 then the solution becomes 
colourless. This is an indication of degraded material. The distance between the specimen's surfaces to the end of the colourless zone is the degraded depth. The degraded level was measured along the cross-section of the specimen's and mean and standard deviation values were obtained. For the case of acidic attack, the degraded depth is visible and thus no phenolphthalein solution was used. The predicted degraded depth based on Equation 2.10 was validated with the measured degraded depth for each degradation mechanism.

\subsection{Results and discussion}

The effect of acidic deterioration and leaching in the ultrasonic response had a major discrepancy. A reflection from the degraded layer was found in the mortar deteriorated by acid [8, 10]. However, a detectable reflection from the degraded layer will not always be present, which is one of the major problems of this methods found in literature [8, 10]. Thus, in this research the focus is on the reflections from both surfaces of the material. This is due to the width of the front wall signal as well as the low reflection coefficient from the back surface with respect to the front surface reflection. In this case, a slight increase in the degradation enabled to resolute both signals.

\subsubsection{Estimated depth from calcium leaching}

Equation 2.10 was solved for two main scenarios. In the first case the two layered structure is considered with only pristine and fully degraded material. A second relevant situation is when the degraded thickness is dominated by the partially degraded and gradually degraded layers. These two scenarios are depicted in Figure 2.8. The total degraded depth shown in the solution of Equation 2.10 is $d_{d}$ only. Thus, the same Equation was solved as the degradation progress through the thickness of the mortar. The parameter $\gamma$ is obtained by the ratio of the pulse velocity at full degradation and pulse velocity at pristine state. Then parameters $\beta$ and $\alpha$ are set from the calculated $\gamma$ in the pristine state. Furthermore, parameters $a$ and $b$ are assumed to be as large as possible $(\approx 0.3)$ in the second scenario whereas in the first scenario these parameters are assumed to be as small as possible $(\approx 0)$.

The change in the acoustic velocity of various degraded levels can be seen in Figure 2.9 and Figure 2.10 for early stage and long deterioration respectively. Only after testing with frequencies in between $0.5 \mathrm{MHz}$ and $1 \mathrm{MHz}$ it was found that the standard deviation for the pristine state is higher for tests performed with lower frequency compared to higher frequency. Additionally, for the lower frequency signals, an increase in the speed of sound in early degraded material was found (see Figure 2.9). Both the high scatter and the increase in speed of sound are due to the resolution problem with low frequency signals. For higher frequency waves this problem does not exist. In Figure 2.9 and Figure 2.10 the two cases shown are: $a=0$ and $a=0.3$. In this research these two conditions are considered as 
the boundaries of the possible leaching events that can happen in a cementitious structure

$$
\begin{aligned}
& D\left\lfloor\left\{\begin{array}{cc}
V_{f d}=\gamma V_{h}=0.54 V_{h} & (f d) \\
V_{g d}=\beta V_{h}=0.8 V_{h} & (g d) \\
V_{p d}=\alpha V_{h}=0.95 V_{h} & (p d) \\
V_{h} & (h)
\end{array} \mid \begin{array}{c}
d_{f d}=c d_{d}=d_{d} \\
d_{g d}=b d_{d}=0 \\
d_{p d}=a d_{d}=0
\end{array}\right\rfloor d_{d}=1\right.
\end{aligned}
$$

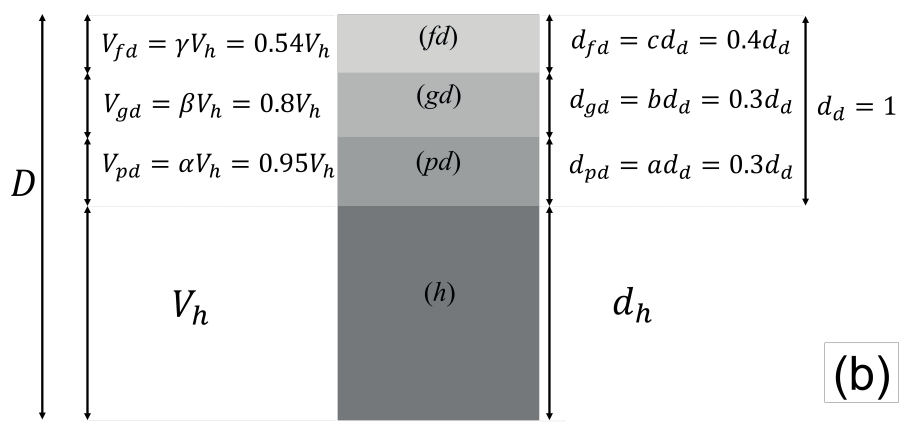

\section{Figure 2.8}

(a) Healthy and fully degraded configuration are present in the structure. (b) Degraded zone dominated by gradually and partially degraded regions. This is considered as a "worst" case where the leaching process is not as sharp and the properties of the material gradually extend from healthy to fully leached.

Figure 2.10 shows the results for long term degradation with $0.5,0.8 \mathrm{MHz}$ and 1 $\mathrm{MHz}$. The back-wall reflection from the $1 \mathrm{MHz}$ central frequency tests were heavily damped after $45 \%$ of degradation depth. The back-wall reflection from the two other frequencies were heavily attenuated after $60 \%$ of degradation depth. In order to characterize larger degradation depths, the specimen's thickness was gradually reduced from the healthy side towards the degraded side by dicing. Thus, instead of inducing further degradation the material is made thinner, resulting in an overall higher degradation. These results are shown in the two darker blue zones in Figure 2.10.

The degradation degree was obtained by dividing the measured degraded depth $\left(d_{d}\right.$, from phenolphthalein tests) with the thickness of the specimen, $D ;\left(d_{d} / D\right) *$ $100 \%$. The results from the ultrasonic tests were correlated with the phenolphthalein tests as shown in Figure 2.10 bottom axis. During this process, it 


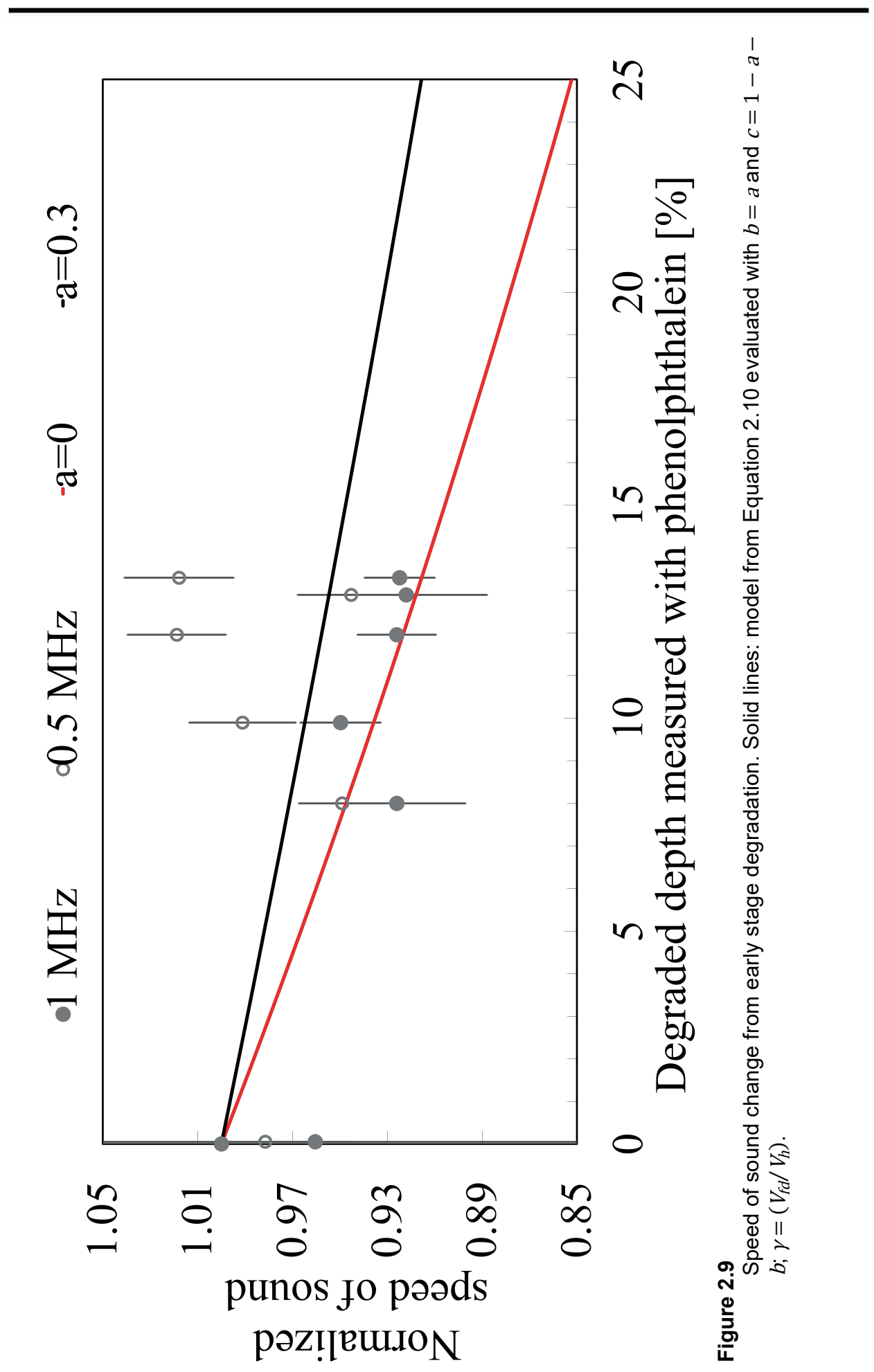




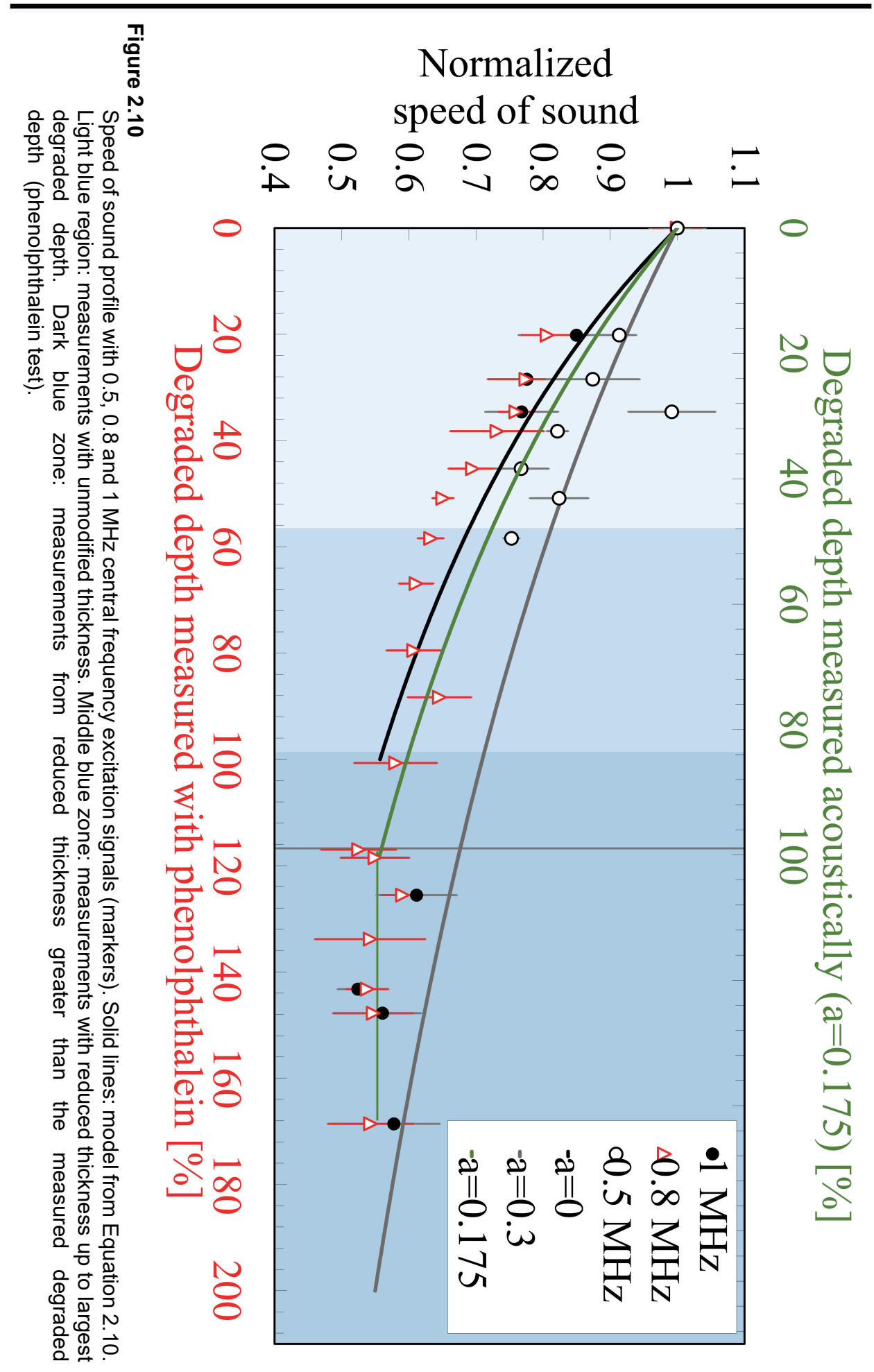


was found that, if the thickness of the specimen was reduced to, where is measured from the phenolphthalein tests, the speed of sound kept decreasing. An apparent degraded depth higher than $100 \%$ is shown in the bottom axis of Figure 2.10. After $117 \%$ of apparent degraded depth, the speed of sound did not drop further. Thus, the fully degraded condition was reached.

On the top axis, the degraded depth obtained from Equation 2.10 when $a=0.175$ shows a $100 \%$ degraded depth when the phenolphthalein results show $117 \%$ (bottom axis). This discrepancy is due to the layers present in the structure. While the phenolphthalein only shows a change in $\mathrm{pH}$ from a threshold value Equation 2.10 shows the fully degraded condition.

\subsubsection{Validation from acidic deterioration}

Figure 2.11 depicts the speed of sound profile for the different degradation depths. The measured speed of sound in fully degraded material due to acidic attack is found to be $1,400 \mathrm{~m} / \mathrm{s}$ with a $0.5 \mathrm{MHz}$ central frequency actuation signal. This is corroborated with the literature $[8,10]$. Due to the heavy attenuation of the waves, new batch of specimens with a lower water-to-cement ratio (0.3) were manufactured and degraded. Experimental results of the lower w/c cement content agree with model from Equation 2.10 evaluated at $a=0$.

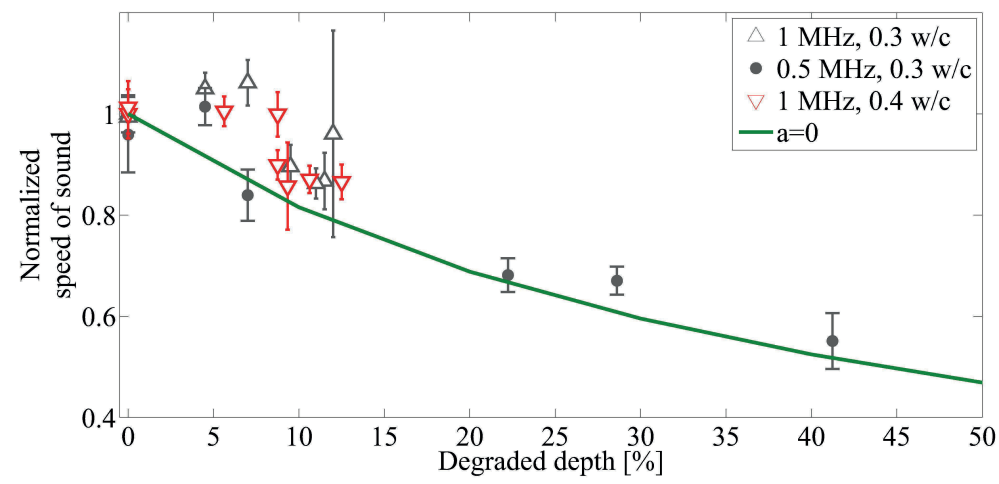

Figure 2.11

Speed of sound profile for the different degraded depths measured with $0.5 \mathrm{MHz}$ and $1 \mathrm{MHz}$ central frequency transducer. Solid line: model from Equation 2.10.

The increase in the speed of sound in the early stage degradation is due to the proximity between the front wall reflection and the reflection from the degraded layer. In Figure 2.12, the process where the front wall is shifted in time to the right as the degraded layer increases. Once the degraded layer is far enough from the front wall reflection, it is shifted back to the initial position as depicted by the dotted lines in the front wall reflection. Furthermore, the back-wall reflection appears to 
increase in amplitude. This is due to the reflection coefficient decrease at the water-degraded interface due to the decomposition of the material.

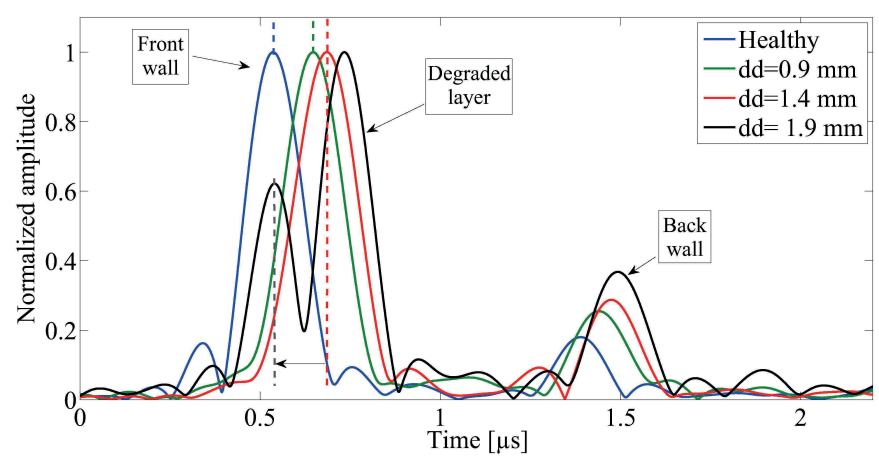

Figure 2.12

Early stage degradation signals from acidic deterioration. Doted lines represent the location in time of the front wall reflection. The data was normalized to the maximum of each signal. Thus, it is not possible to see the reduction in amplitude from the front wall. In the legend, $d_{d}$ is degraded depth

\subsubsection{Response at acoustic interfaces}

The amplitude from the reflection at the water-degraded mortar interface (front wall) change as the degradation level increases. At very early stage there is high variation in the amplitude due to the incomplete degradation at the surface as shown in the white region from Figure 2.13. At further degradation levels, the acoustic interface mismatch does not change as full degradation is present at the surface (see the blue zone in Figure 2.13). On the other hand, the amplitude of the mortar-water interface (back wall) does not change as the back side of the specimen remains healthy (non-degraded). Only when the degradation depth has penetrated the full thickness there will be an effect on the interface mismatch at the back of the specimen. The decrease of amplitude at the first interface (front wall) during early stage degradation means that more energy is transmitted to the material (see Figure 2.13). This effect is frequency dependent. The higher the excitation frequency the higher the reduction in front wall and back wall amplitudes. In Figure 2.13, a gradual transition between early degradation and long degradation is seen from the white zone to the blue zone. The amplitude of the pristine state for both front wall and back wall was considered as the reference for calculating the $\mathrm{dB}$ change shown in Figure 2.13.

For the case of acidic deterioration, the attenuation increased as the degradation depth increased. In this case, in the early stage degradation there is no increase in the back-wall reflection amplitude. The water/degraded reflection coefficient remains constant as soon as degradation is present in the material and this does not further change with respect to the degraded depth. The determination of the 


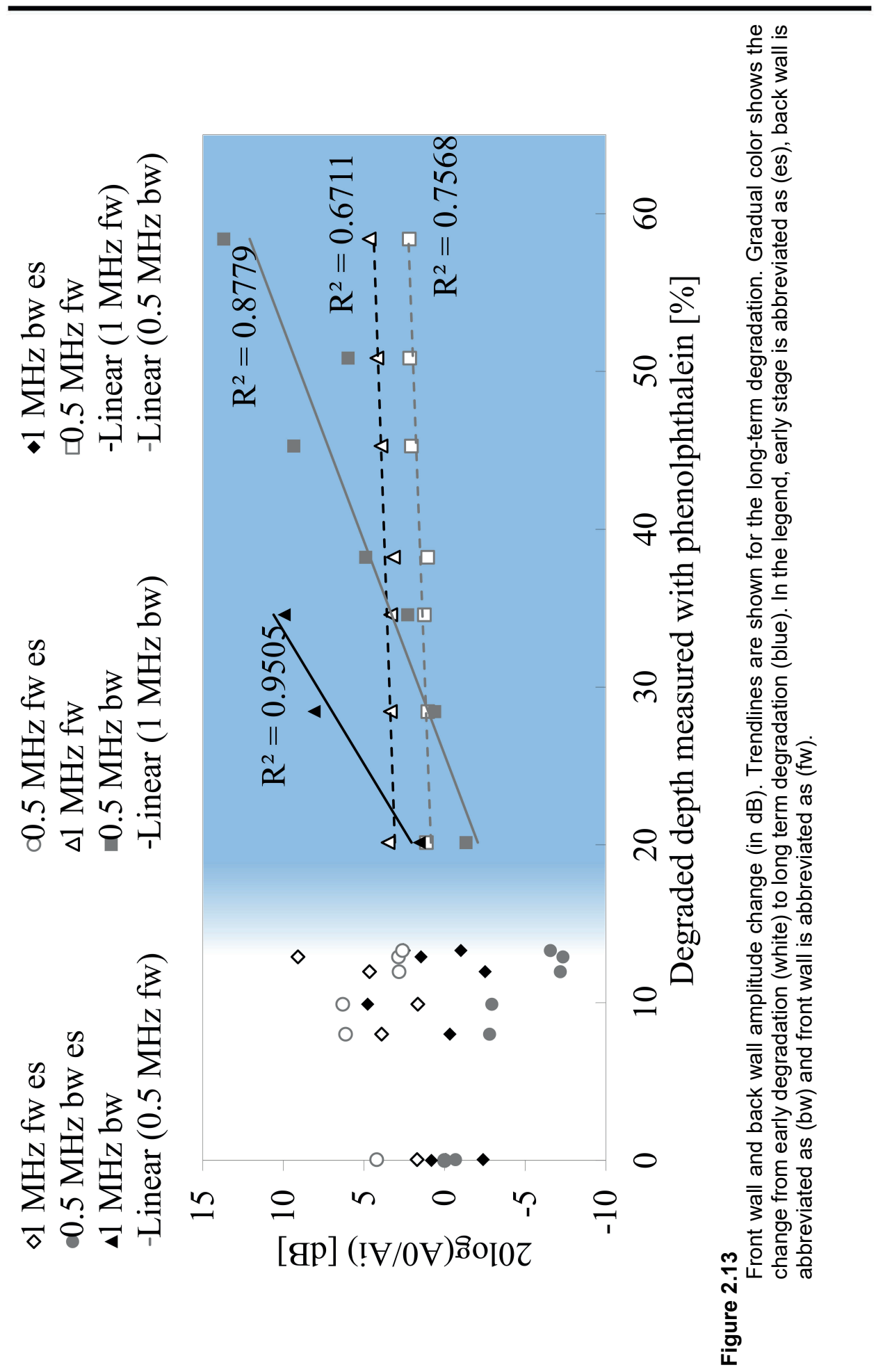


amount of degradation based on Equation 2.10 had a similar response compared with the correlation between the acoustic and non-acoustic measurements. The speed of sound decreased as the degradation depth increased. In some cases, the experimental results showed an increase in pulse velocity, especially in the very early stage, and the decrease rate of speed of sound as the degraded depth increases was different.

There are many factors that are attributed to these discrepancies. For instance, in the early stage acidic attack, the proximity between the front wall and degraded layer reflections caused an apparent increase in the speed of sound. After $10 \%$ of degraded depth, Equation 2.10 and the experimental results show a good agreement. The case of calcium leaching has a different story. This is mostly because the degraded zone is not as sharp as in the acidic deterioration (see Figure 2.1). In Figure 2.14, the transition between degraded and non-degraded is not very clear. The purple indicator gradually changes. In the experimental part, the degraded layer was considered until where the blue arrow in Figure 2.14 is pointing. However, the identification from the purple coloration of the complete degraded zone was not possible to achieve. Thus, the results from the calcium leaching should have a higher degraded depth for the measured pulse velocity.

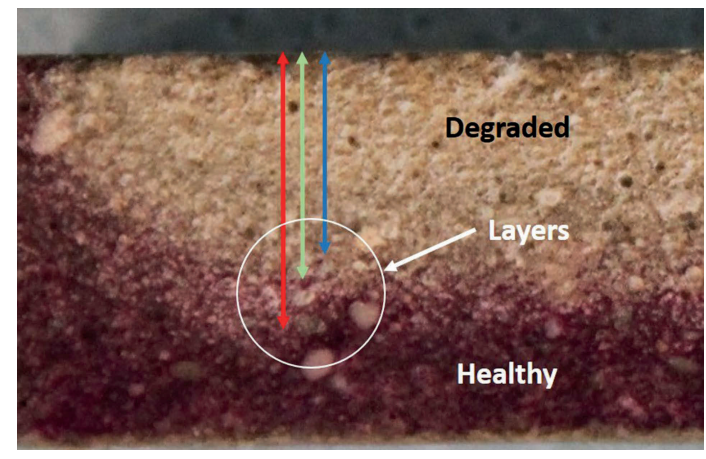

Figure 2.14

Cross-section view of a mortar degraded from contact with ammonium nitrate solution. Purple color represents high pH (pristine). Grey zone represents low pH (degraded).

As mentioned in previous section, Equation 2.10 is a distribution of material properties (including speed of sound) and thicknesses of layers within the mortar. However, the response of the reflections from a layered structure from a wave propagation approach are different. For instance, the increase in pulse velocity from early acidic deterioration. The assumption that the distribution of the degraded layers starts from a sharp transition to an extended gradual change in the material properties is something to investigate as well. The distribution of the layers is a complex phenomenon which is dependent on many factors such as: porosity, cement ratio, manufacturing procedure, additives, geometry as well as the characteristics of the aggressive solution. 
A final source of discrepancy between the experimental work and the solution of Equation 2.10 is the determination of the time-delay of the reflections of interests. The error was more prominent in the long degradation from calcium leaching where the standard deviation increases. The back-wall reflection was at the level of the noise, thus increasing the error in the detection of the time delay.

\subsection{Conclusion}

The amount of degradation in a cementitious structure can be determined with the methodology presented for both types of degradation mechanisms. It is important that the pristine condition and the fully degraded state are known. In this research the long-term degradation from drinking water pipes was represented by accelerated chemical deterioration in mortar specimens. The manufactured specimens had high porosity and low compaction which lead to high permeability. The degraded layer was then composed by the partially, gradually and fully degraded zones. Even though, there was a difference between the destructive and the acoustic method, this can be accounted for when the fully degraded material is characterized. With the Equation 2.10 the amount of degraded material can be quantified. Accurate degradation depth quantification in very early stage $(<8 \%)$ may not be possible, however, detection of very early stage $(<5 \%)$ is possible as in difference with previous research. Despite the discrepancy found between the experimental work and the solution of Equation 2.10 it is believed that the solution of the latter has more accuracy than the measured degraded depth from the phenolphthalein tests. Which in a practical application this is more beneficial avoiding destructive testing.

With a combination of back wall amplitude and pulse velocity it is possible to determine the degraded level in the structure. The amplitude of the reflected waves at the surfaces of the structure provides also important information for the early degradation level. Smaller measurement wavelengths are more sensitive to degradation compared to higher frequencies. Thus, the actuation frequency has to be selected depending on the initial thickness of the specimen. In this research, the most suitable excitation frequency for a $20 \mathrm{~mm}$ thickness structure was $0.8 \mathrm{MHz}$. This allowed to detect up to $60 \%$ degradation level. For very early degraded depth, higher frequencies may be used. In the methodology presented it is vital that the acoustic measurements are correlated with the real measured thickness in order to characterize the pulse velocity as a function of the degraded depth. But also, to determine whether the structure is dominated by a fully degraded layer or if it is composed gradual and partial change of the calcium content. This is a subject of further studies.

\section{ACKNOWLEDGMENT}

This work was performed in the cooperation framework of Wetsus, European Centre of Excellence for Sustainable Water Technology (www.wetsus.eu). Wetsus is co-funded by the Dutch Ministry of Economic Affairs and Ministry of Infrastructure and Environment, the Province of Fryslân, and the Northern 
Netherlands Provinces. The authors like to thank the participants of the research theme "Smart Water Grids" for the fruitful discussions and their financial support. A special acknowledgement to Acquaint B.V. for its support throughout the development of this work. 


\section{REFERENCES}

1. R. Chowdhury, Y. Hu, and D. Wang, 'Condition Evaluation of Asbestos Cement Water Mains,' in Pipelines 2012, 2012, no. 306, pp. 288-297.

2. D. Wang and D. R. Cullimore, 'Bacteriological challenges to asbestos cement water distribution pipelines,' J. Environ. Sci., vol. 22, no. 8, pp. 1203-1208, 2010.

3. D. Wang, R. Cullimore, Y. Hu, and R. Chowdhury, 'Biodeterioration of asbestos cement (AC) pipe in drinking water distribution systems,' Int. Biodeterior. Biodegrad., vol. 65, no. 6, pp. 810-817, 2011.

4. F. Bouchaala, C. Payan, V. Garnier, and J. P. Balayssac, 'Carbonation assessment in concrete by nonlinear ultrasound,' Cem. Concr. Res., vol. 41, no. 5, pp. 557-559, 2011.

5. C. Carde and R. François, 'Effect of the leaching of calcium hydroxide from cement paste on mechanical and physical properties,' Cem. Concr. Res., vol. 27, no. 4, pp. 539-550, 1997.

6. T. De Larrard, F. Benboudjema, J. B. Colliat, J. M. Torrenti, and F. Deleruyelle, 'Concrete calcium leaching at variable temperature: Experimental data and numerical model inverse identification,' Comput. Mater. Sci., vol. 49, no. 1, pp. 35-45, 2010.

7. J. J. Gaitero, J. S. Dolado, C. Neuen, F. Heber, and E. A. B. Koenders, '3D Computational Simulation of Calcium Leaching in Cement Matrices,' Mater. Construcción, vol. 64, no. 316, p. e035, 2014.

8. A. Demčenko, H. A. Visser, and R. Akkerman, 'Ultrasonic measurements of undamaged concrete layer thickness in a deteriorated concrete structure,' NDT E Int., vol. 77, pp. 63-72, 2016.

9. S. Ould Naffa, M. Goueygou, B. Piwakowski, and F. Buyle-Bodin, 'Detection of chemical damage in concrete using ultrasound.', Ultrasonics, vol. 40, no. 1-8, pp. 247-51, 2002.

10. H. H. Delgadillo, R. Loendersloot, R. Akkerman, and D. Yntema, 'Development of an inline water mains inspection tech nology,' in 2016 IEEE International Ultrasonics Symposium (IUS), 2016, vol. 2016-Novem, pp. 1-4.

11. S. K. Ramamoorthy, Y. Kane, and J. a Turner, 'Ultrasound diffusion for crack depth determination in concrete.,' J. Acoust. Soc. Am., vol. 115, no. 2, pp. 523-529, 2004.

12. J. Becker, L. J. Jacobs, and J. Qu, 'Characterization of Cement-Based Materials Using Diffuse Ultrasound,' J. Eng. Mech., vol. 129, no. 12, pp. 14781484, Dec. 2003.

13. J. Carette and S. Staquet, 'Monitoring the setting process of mortars by ultrasonic $\mathrm{P}$ and $\mathrm{S}$-wave transmission velocity measurement,' Constr. Build. Mater., vol. 94, pp. 196-208, 2015.

14. P. Antonaci, C. L. E. Bruno, A. S. Gliozzi, and M. Scalerandi, 'Monitoring evolution of compressive damage in concrete with linear and nonlinear ultrasonic methods,' Cem. Concr. Res., vol. 40, no. 7, pp. 1106-1113, 2010.

15. M. G. Hernández, M. a G. Izquierdo, A. Ibáñez, J. J. Anaya, and L. G. Ullate, 'Porosity estimation of concrete by ultrasonic NDE,' Ultrasonics, vol. 38, no. 1, pp. 531-533, 2000. 
16. D. G. Aggelis and T. P. Philippidis, 'Ultrasonic wave dispersion and attenuation in fresh mortar,' NDT E Int., vol. 37, no. 8, pp. 617-631, Dec. 2004.

17. D. G. Aggelis and T. Shiotani, 'Surface wave dispersion in cement-based media: Inclusion size effect,' NDT E Int., vol. 41, no. 5, pp. 319-325, Jul. 2008.

18. D. G. Aggelis, S. Momoki, and H. Chai, 'Surface wave dispersion in large concrete structures,' NDT E Int., vol. 42, no. 4, pp. 304-307, Jun. 2009.

19. H. Sun and J. Zhu, 'Monitoring Early Age Properties of Cementitious Material Using Ultrasonic Guided Waves in Embedded Rebar,' J. Nondestruct. Eval., vol. 36, no. 1, p. 5, Mar. 2017.

20. M. S. J. Gan, Cement and concrete. CRC Press, 1997.

21. S. Chandra, 'Hydrochloric acid attack on cement mortar - An analytical study,' Cem. Concr. Res., vol. 18, no. 2, pp. 193-203, Mar. 1988.

22. V. Zivica and A. Bajza, 'Acidic attack of cement based materials -- a review. Part 1. Principle of acidic attack,' Constr. Build. Mater., vol. 15, pp. 331-340, 2001.

23. L. De Ceukelaire, 'The effects of hydrochloric acid on mortar,' Cem. Concr. Res., vol. 22, no. 5, pp. 903-914, Sep. 1992.

24. R. K. Walter, P. H. Lin, M. Edwards, and R. E. Richardson, 'Investigation of factors affecting the accumulation of vinyl chloride in polyvinyl chloride piping used in drinking water distribution systems,' Water Res., vol. 45, no. 8, pp. 2607-2615, 2011.

25. I. Rosborg, B. Nihlgård, and M. Ferrante, 'Mineral Composition of Drinking Water and Daily Uptake,' in Drinking Water Minerals and Mineral Balance, Cham: Springer International Publishing, 2015, pp. 25-31.

26. M. Mainguy, C. Tognazzi, J.-M. Torrenti, and F. Adenot, 'Modelling of leaching in pure cement paste and mortar,' Cem. Concr. Res., vol. 30, no. 1, pp. 8390, Jan. 2000.

27. V. H. Nguyen, H. Colina, J. M. Torrenti, C. Boulay, and B. Nedjar, 'Chemomechanical coupling behaviour of leached concrete,' Nucl. Eng. Des., vol. 237, no. 20-21, pp. 2083-2089, Nov. 2007.

28. N. Burlion, D. Bernard, and D. Chen, 'X-ray microtomography: Application to microstructure analysis of a cementitious material during leaching process,' Cem. Concr. Res., vol. 36, no. 2, pp. 346-357, 2006.

29. C. Carde and R. François, 'Modelling the loss of strength and porosity increase due to the leaching of cement pastes,' Cem. Concr. Compos., vol. 21, no. 3, pp. 181-188, 1999.

30. C. Carde, R. François, and J.-M. Torrenti, 'Leaching of both calcium hydroxide and C-S-H from cement paste: Modeling the mechanical behavior,' Cem. Concr. Res., vol. 26, no. 8, pp. 1257-1268, Aug. 1996.

31. G. Villain, V. Garnier, Z. M. Sbartaï, X. Dérobert, and J.-P. Balayssac, 'Development of a calibration methodology to improve the on-site nondestructive evaluation of concrete durability indicators,' Mater. Struct., vol. 51, no. 2, p. 40, Apr. 2018.

32. S. P. Neal and D. O. Thompson, 'A prior knowledge based optimal Wiener filtering approach to ultrasonic scattering amplitude estimation,' J. Acoust. Soc. Am., vol. 86, no. S1, pp. S94-S94, Nov. 1989. 
33. F. Honarvar, H. Sheikhzadeh, M. Moles, and A. N. Sinclair, 'Improving the time-resolution and signal-to-noise ratio of ultrasonic NDE signals,' Ultrasonics, vol. 41, no. 9, pp. 755-763, Mar. 2004.

34. G. Hayward and J. E. Lewis, 'Comparison of some non-adaptive deconvolution techniques for resolution enhancement of ultrasonic data,' Ultrasonics, vol. 27, no. 3, pp. 155-164, May 1989.

35. S. P. Neal, P. L. Speckman, and M. A. Enright, 'Flaw signature estimation in ultrasonic nondestructive evaluation using the Wiener filter with limited prior information,' IEEE Trans. Ultrason. Ferroelectr. Freq. Control, vol. 40, no. 4, pp. 347-353, Jul. 1993.

36. E. Oruklu, Y. Lu, and J. Saniie, 'Hilbert transform pitfalls and solutions for ultrasonic NDE applications,' in 2009 IEEE International Ultrasonics Symposium, 2009, pp. 2004-2007.

37. Z. Lu, 'Estimating Time-of-Flight of Multi-superimposed Ultrasonic Echo Signal through Envelope,' in 2014 International Conference on Computational Intelligence and Communication Networks, 2014, pp. 300-303.

38. P. M. Gammell, 'Improved ultrasonic detection using the analytic signal magnitude,' Ultrasonics, vol. 19, no. 2, pp. 73-76, Mar. 1981.

39. B. Audoin and J. Roux, 'An innovative application of the Hilbert transform to time delay estimation of overlapped ultrasonic echoes,' Ultrasonics, vol. 34, no. 1, pp. 25-33, Mar. 1996.

40. N. E. Huang and N. O. Attoh-Okine, Eds., The Hilbert-Huang Transform in Engineering. CRC Press, 2005. 


\title{
Chapter 3
}

\section{Ultrasonic inline inspection of a cement- based drinking water pipeline}

\begin{abstract}
A method to process ultrasonic signals from an inline inspection in a cementbased drinking water main is proposed. The data was obtained from an inspection performed in a Dutch drinking main section. The data is processed in two major steps. Firstly, the parameters that provide the condition of the cement are extracted. Secondly, images of the degradation within the pipes of the inspected trajectory were generated. The main contributions in this chapter are (i) the confirmation that cement-based pipes can be inspected and evaluated with the ultrasonic pulse echo technique and (ii) the upscaling of the processing method in an automated manner for visualization of the degraded condition. Lastly, a sensitivity study of the parameters relevant to the determination of the degraded depth has been performed. The speed of sound in cement appear to be the most relevant parameter.
\end{abstract}

This chapter has been submitted as: Delgadillo, H. H., Geelen, C., Kakes, R., Loendersloot, R., Yntema, D., Tinga, T., \& Akkerman, R. (2019). Ultrasonic inline inspection of a cement-based drinking water pipeline. Engineering Structures. 


\subsection{Introduction}

Preventing failure of drinking water distribution systems is a current interest in many countries around the world. Repairing a failed pipe can cost tens of thousands of Euros. What is more, interruption of the supplied water and thirdparty damages are further consequences of a failed pipe. This raises the question of how to prevent failure in the drinking water network. The replacement of the mains can be performed effectively if the state of the pipe is known. In this research, a methodology that can detect and determine the degraded thickness in a cementitious structure is applied to a real cement-based drinking water pipe.

\subsubsection{Commercially available inspection technologies}

Cementitious pipes account for nearly $30 \%$ of the Dutch drinking water network [1]. In today's condition assessment market, there are two non-destructive inspection methods that have been used to determine the degradation in cementbased pipes. These are the ePulse technology and surface penetrating radar (SPR) [2-11]. None of them is versatile enough to be used in a routine fashion nor provide information on the complete pipe section. Inspection of the water mains should preferably be done in a non-destructive way, not requiring the pipes to be dug out and with high resolution information.

A recent study showed a methodology to quantify chemical degradation throughthickness in cementitious structures based on high frequency sound waves [12]. Quantification of degradation is done by measuring the acoustic pulse velocity through the thickness of the material. However, the pipe's thickness and speed of sound in pristine and fully degraded conditions have to be known [12, 13]. This might not be the case in a real inspection.

In collaboration with Acquaint B.V., an inspection company, the inspection data from an inspected section from the Dutch drinking water grid was used. The objectives of this research are firstly to find the relevant parameters from the acoustic data in a real cement-based drinking water pipeline. The second objective is to provide a processing method for the upscaling of the parameter extraction and the visualization of the estimated degraded depth. Finally, the sensitivity of the parameters that can influence the estimation of the degraded depth is investigated.

\subsubsection{Leaching depth in a degraded structure}

In a two-layered cementitious structure, it is possible to determine the thickness of the degraded layer based on the time-of-flight of the acoustic waves. The degradation of cement-based structures generates a difference in material properties from the external surfaces towards the inside of the material. Extensive information on chemical deterioration of cementitious structures can be found in 
$[3,11,14-19]$. The degraded zone is shown as $d$ in Figure $3.1, h$ is the healthy zone and $D$ is the total wall thickness.

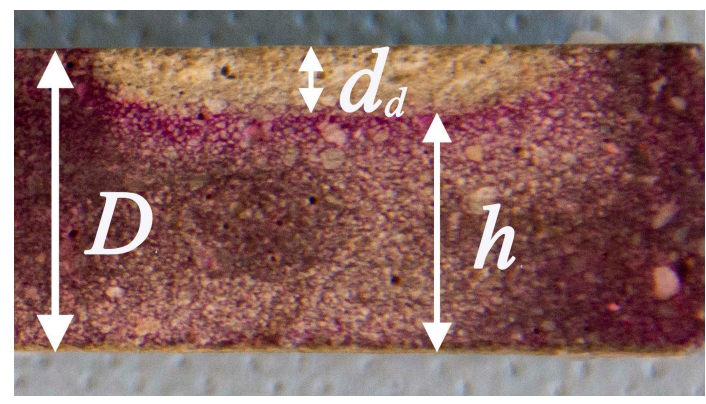

Figure 3.1

Leached cementitious structure treated with phenolphthalein to determine $\mathrm{pH}$ value. The colourless region $(d)$ represents the degraded material (low $\mathrm{pH})$ and the purple region represents non-degraded material ( $\mathrm{pH}$ still high enough).

If the speed of sound in pristine and fully degraded condition is known, the thickness of the degraded layer is determined as

$$
d_{d}=\frac{D}{V_{D}}\left(\frac{V_{h}-V_{D}}{\frac{1}{\gamma}-1}\right)
$$

where $D$ is the thickness of the specimen, $V_{h}$ is the speed of sound in pristine cement, $V_{D}$ is the measured speed of sound across the thickness and $\gamma$ is a constant that relates the speeds of sound in the pristine and in degraded cement [12].

\subsection{Methodology}

This section is divided in three main parts. In the first part, details on the performed inspection, including the trajectory and specifications of the inspection tool, will be given. Then, the principle of the ultrasonic tests together with the processing of the signals from ultrasonic raw responses to time-of-flight information is discussed. The third part, the visualization section, focuses on handling the extracted parameters such that the degraded state can be understood qualitatively and quantitatively.

\subsubsection{Inspected trajectory}

A cement-based pipeline was inspected with an inspection tool equipped with eight ultrasonic sensors. The tool is made of a cylindrical foam (black colour in Figure $3.2 \mathrm{a}$ and Figure $3.2 \mathrm{~b}$ ) that is compressible to the inner diameter of the pipe. 
It is equipped with eight ultrasonic transducers as depicted in Figure 3.2a. Each sensor performs a measurement every 0.05 seconds. The tool translates through the pipe at a speed of approximately $c=0.2 \mathrm{~m} / \mathrm{s}$. The tool obtains 160 measurements per second and approximately 800 measurements per meter of inspected water main trajectory.

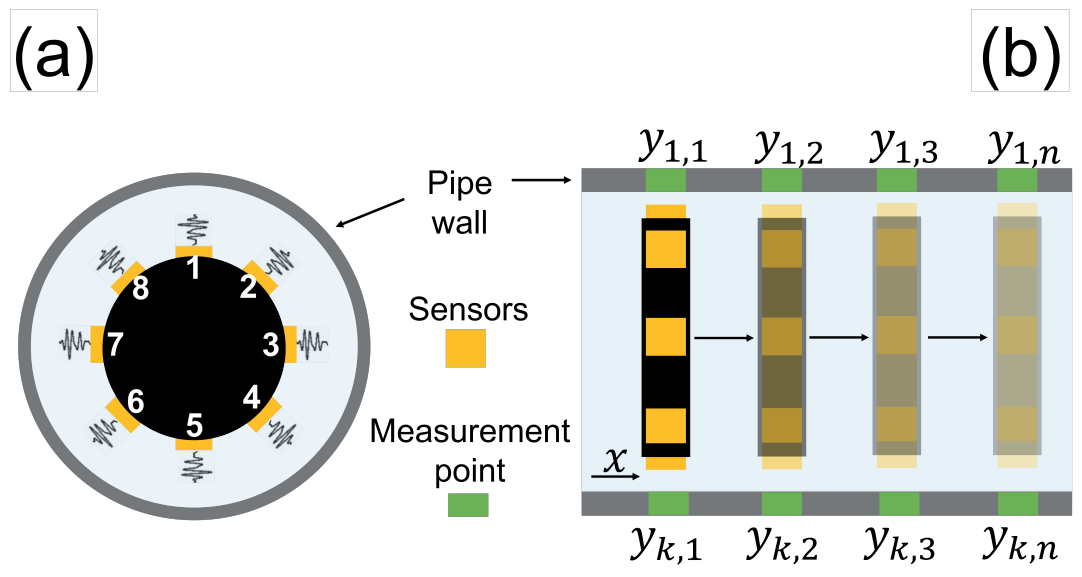

Figure 3.2

Schematic representation of the inspection tool with front view (left) and side view (right). The tool carries 8 ultrasonic transducers along the circumference. Each sensor transmits acoustic waves through the water, that propagate through the thickness of the pipe, and reflects back to the transducer, resulting in measurements at locations $y_{k, n}$

The face of the ultrasonic transducers is aligned parallel to the internal surface of the pipe (see Figure 3.2a). Each transducer is excited by an electric pulse which in turns converts electrical energy into mechanical energy (acoustic waves). These waves travel through the water (as shown in Figure 3.2a).

The transition from water-to-inner-surface and outside-surface-to-soil interfaces produce energy transmission and reflection (see Figure 3.3). The energy that is reflected at the water-to-inner-surface interface is called the front wall reflection (see Figure 3.3). In this research referred to as front wall (FW) and back wall (BW) reflections, respectively. The transmitted acoustic waves then travel through the thickness of the specimen. At the outside-surface-to-soil interface, most of the energy is reflected and some energy is transmitted to the surrounding soil.

The energy that is reflected at the latter interface is called the back-wall reflection (see Figure 3.3). The inspection was done in 2018 and its geographical location is shown in Figure 3.4 (green line). The inspected trajectory length is $1939 \mathrm{~m}$. The tool has a location tracker, such that the inspected trajectory can be visualized on a map as shown in Figure 3.4. 

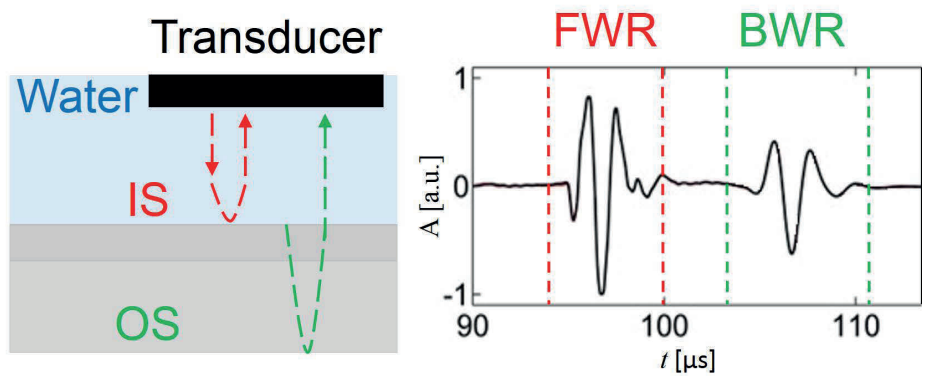

Figure 3.3

Reflection of sound waves at the inner surface (IS) and outer surface (OS) of the pipe.

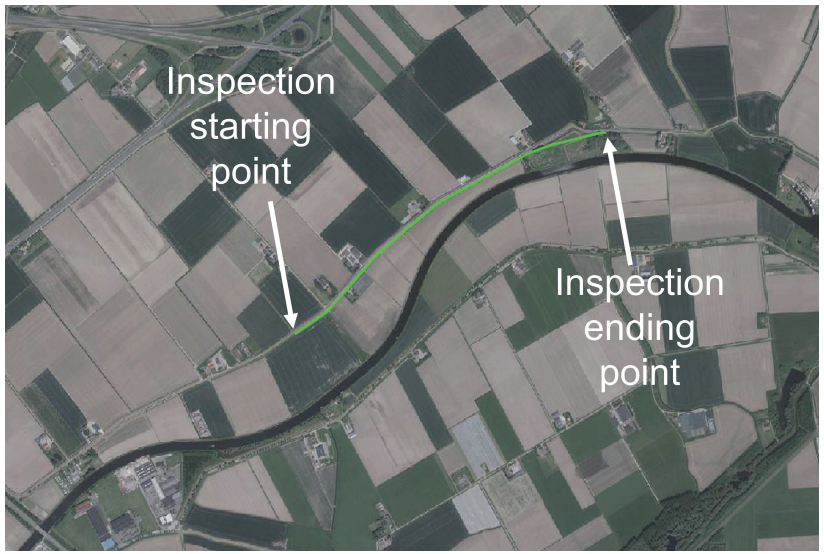

Figure 3.4

Aerial view of inspected pipeline section

\subsubsection{Automatic processing of ultrasonic signals}

The objective of data processing is here to translate the obtained raw ultrasonic signals to a parameter related to the amount of degradation. This is achieved by estimating the time difference $\Delta t$ between front and back wall time-of-arrival (see Figure 3.3). The processing of the acquired data is performed with the same scheme as presented in $[12,13]$.

With the vast amount of data being processed, automation is necessary. The front wall and back wall reflections are automatically detected with the following process. First, the absolute magnitude of the derivative of the entire recorded signal is calculated (see Figure $3.5 \mathrm{~b}$ ). Then, the maximum derivative magnitude is located (black point in Figure 3.5b). Based on the present pipe thickness, acquisition rate and central frequency of the sensor, $6 \%$ of the total signal length is selected (vertical black lines in Figure 3.5a) avoiding overlapping with other 
nearby reflections. The signal within the selected $6 \%$ is zero padded (i.e. zero values are added to create a convenient sample size) as shown by the horizontal line in Figure 3.5b. Thereafter, a second maximum derivative is located (red point in Figure 3.5b). Then the two selected signals (signal within the vertical black and red lines in Figure 3.5a) are extracted.

Taking the derivative of the signal "filters" out parts of the signal such as 'ringing' from the excitation pulse. Removing ringing from the excitation pulse allows to only select the reflections of interests in a robust manner. To further process the selected reflections (front wall and back wall) a band-pass filter is used. The filter scheme and process is shown in $[12,13]$. Finally, the difference between the timeof-arrival of the FW and BW signals $(\Delta t)$ is calculated. Equation 3.1 is then solved by estimating $V_{D}$ from the measured $\Delta t$. The following assumptions are made in this analysis: (i) throughout the trajectory the wall thickness is constant; (ii) degradation is mostly a general process, thus local changes are not expected; (iii) and degradation is homogeneous along the pipe circumference.

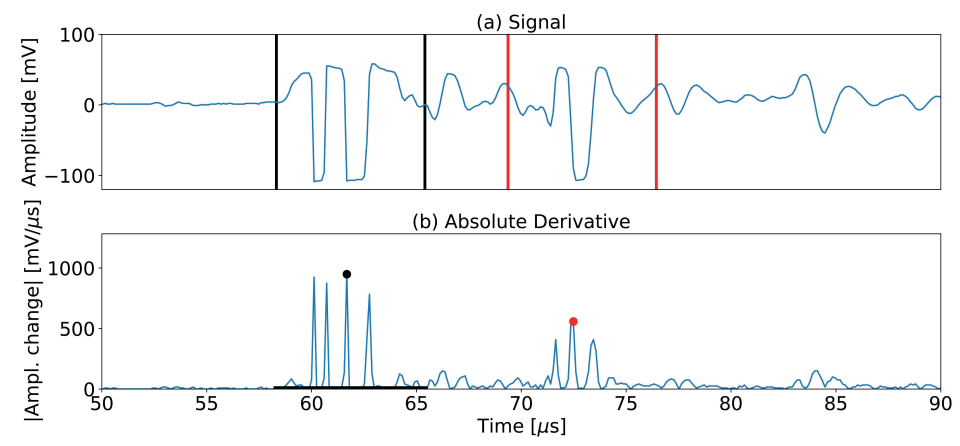

Figure 3.5

Signal processing scheme. (a) Time signal with the selected limits for the extraction of the front wall (black lines) and back wall (red lines); (b) Absolute derivative with the zero padded section (bottom black line) and the maximum amplitude of front wall (black point) and maximum amplitude of back wall (red point).

\subsubsection{Visualization of extracted parameters}

Large variations on the detected degradation depth are expected, and can arise due to several reasons: the joints between pipes, low amplitude reflections, low signal-to-noise ratio and inadequate signal filtering. The latter is a direct effect of low amplitude reflections which are not properly detected by the signal processing. A moving average filter was used on the calculated time-of-flight difference $(\Delta t)$ of each sensor separately. Assuming a uniform degradation along the pipe circumference, the data from all eight sensors was averaged. For each measurement location, three values were extracted: the maximum, the minimum and the average of the time difference $\Delta t$. Finally, the degraded depth was calculated from Equation 3.1. The calculated degraded depth will be shown in an 
aerial view of the trajectory, similar to the one in Figure 3.4. This facilitates the localization of spots that need attention from the water supply company.

\subsection{Results and discussion}

\subsubsection{Visualization of extracted parameters}

A boxplot depicting the time-of-flight difference $\Delta t$ per sensor over the complete inspected trajectory is shown in Figure 3.6. The mean and median values are very similar in all sensors, which indicates a normal distribution of the degradation along the pipe circumference. This validates the proposed averaging over the eight sensors per measurement point.

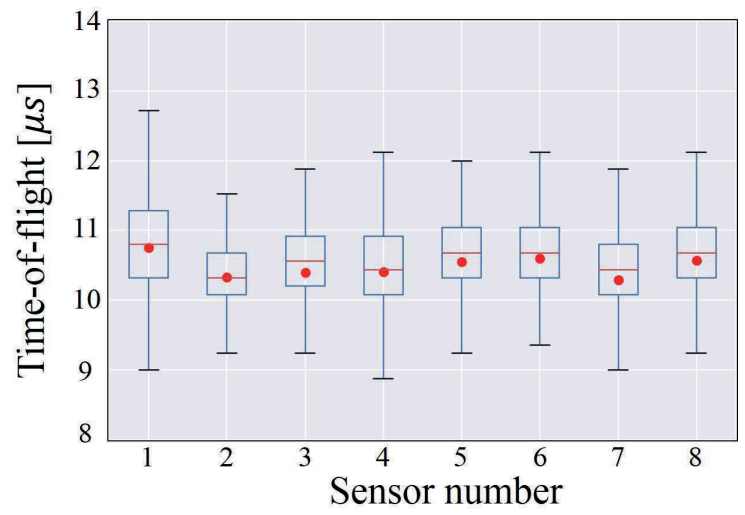

Figure 3.6

Boxplot of the time of flight variation over the inspected trajectory with the median (red line) and average (red dot) values per sensor. The black lines represent the $75 \%$ quartiles and the boxes represent the $25 \%$ quartiles.

The outcome of the data processing is shown in Figure 3.7. In the top figure three different lines of data are shown ( $i$ ) the light blue line depicts the time difference $\Delta t$ for one sensor without processing, (ii) the dark blue line depicts the time difference $\Delta t$ after application of a moving average for one sensor and (iii) the red line represents the average of the eight sensors, again after application of moving average to each of them. The colour map in the lower part of Figure 3.7 shows the time difference $\Delta t$ for each individual sensor. The blue vertical lines in the figure on the top represent the joints between pipe sections (the joints are in between two closely spaced lines). In Figure 3.7 the $x$ axis for both parts of the figure represent the distance along the inspected trajectory.

The time difference indicated by the red line (average over 8 sensors) is slightly higher in comparison with the time difference indicated by the dark blue line (single sensor) from $625 \mathrm{~m}$ to $630 \mathrm{~m}$ (see Figure 3.7). On the contrary, single sensor time difference is slightly higher than the averaged sensor time difference from $620 \mathrm{~m}$ 

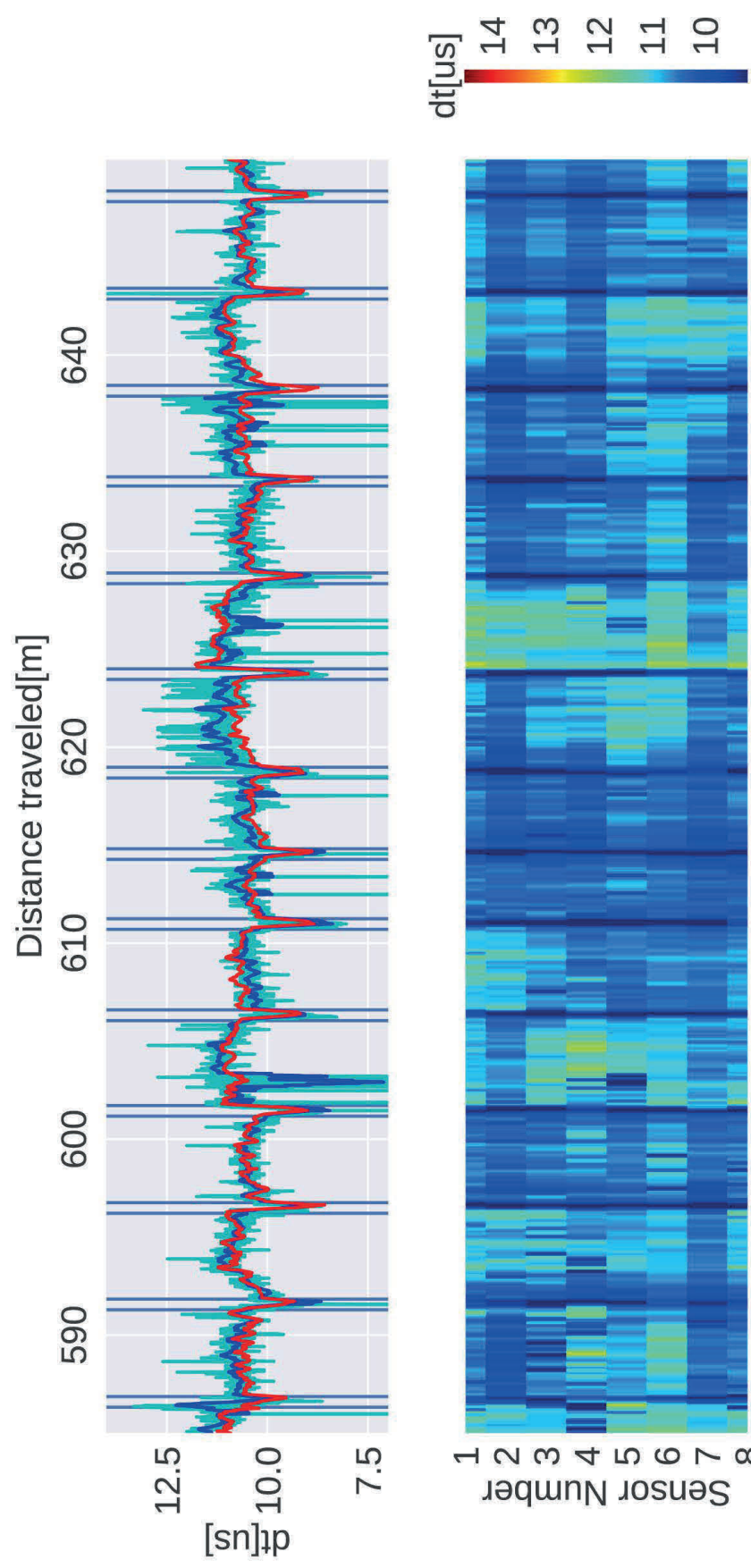

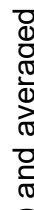
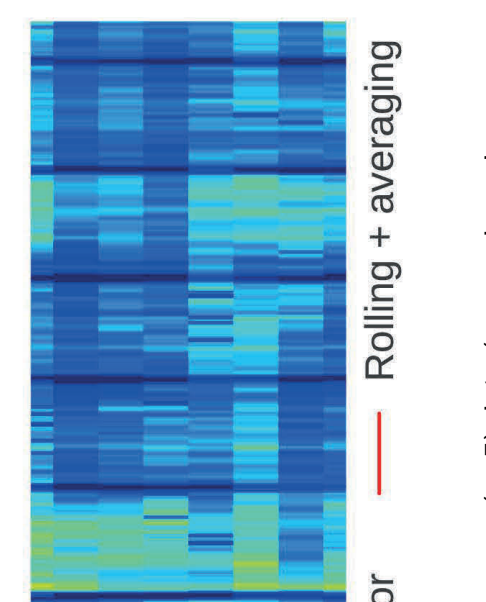

ธัँ

(1)

ऐ)

孚

ह ํํ

음 옥

उั ह

눅

劳

की

डั

市

की

○ $\quad$ के

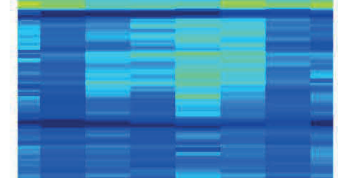

这

ज क ल

인.들

言 䎡 음

कह

흐

음응

ㄷำ

요

음 음

क

ญ 늠

ฮे

힝으

曹

훙

등.

总

पू

崩

¿

든 옹

$\stackrel{\sqrt[N]{N}}{\frac{1}{N}}$

$\hat{m}^{\stackrel{\frac{0}{5}}{\frac{\pi}{7}} \frac{\pi}{\pi}}$

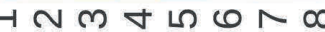
נəqUnN IOSUOS

온 
to $625 \mathrm{~m}$ (see Figure 3.7). This effect is due to the averaging over the eight sensors. For both cases, the colour map reflects the real time difference at a particular location for a particular sensor. Thus, the averaged sensor time difference in the top figure in Figure 3.7 serves as a guide for detecting major changes in the time difference throughout the inspected trajectory. The inclusion of the maximum and minimum sensor values is proposed in this research as well.

The degraded depth is estimated based on Equation 3.1, assuming a constant total wall thickness. With the processing scheme presented in this research, the ultrasonic data can be visualized in a way that provides the optimal information to evaluate the condition of the cement-based pipeline. As shown in Figure 3.7, the change in time difference between front wall and back wall along the pipe length is clearly identified. Moreover, a very important step in this research is the upscaling of the methodology from a laboratory environment to a real pipeline inspection. The validity of the proposed pre-processing procedure will be shown in the next subsection.

\subsubsection{Results of inspected section}

The average degradation depth per measurement location was used to generate one quantitative degradation depth variation over the inspected trajectory. This is an estimate of the amount of leaching in the pipe at that specific measured point. The leaching depth is estimated assuming a constant pipe wall thickness. The degraded depth values are shown for segments of $100 \mathrm{~m}$. Assuming a fairly homogeneous acoustic velocity in its pristine state, the degraded depth can only be attributed to chemical degradation. However, throughout the trajectory, certain pipe sections show a behaviour that is inconsistent with these assumptions. Four examples of these inconsistent events are discussed to describe the phenomena responsible for aberrant degraded depth measurements.

\section{(I) Sudden degraded depth change between neighbouring pipes}

In Figure 3.8 at $455 \mathrm{~m}$, a sudden increase in degradation depth between to the left and right neighbouring pipe sections can be seen. If the assumption that the thickness is constant throughout the inspected trajectory is true, it would mean that a $6 \mathrm{~mm}$ degradation difference exists between the pipe at $455 \mathrm{~m}$ and the two pipes next to it. The opposite behaviour can be seen at $460 \mathrm{~m}$ (the pipe contiguous to the one with the highest degraded depth).

The significant and abrupt changes in degradation depth in these locations can be explained in three ways. Firstly, considering the assumption that the pipe wall thickness is constant, the pipe with the higher degradation would have to be buried in soil or convey water that is more aggressive than at its neighbours on the right and on the left. Thus, it would be a very special case were only that specific pipe has severe damage. A second reason, and still considering the assumption that the thickness is constant, is that the pipe with the lowest degradation has already been replaced before, and thus is actually "healthier". For the case at $460 \mathrm{~m}$ (low degraded depth), the latter seems realistic as there is only one pipe section that 


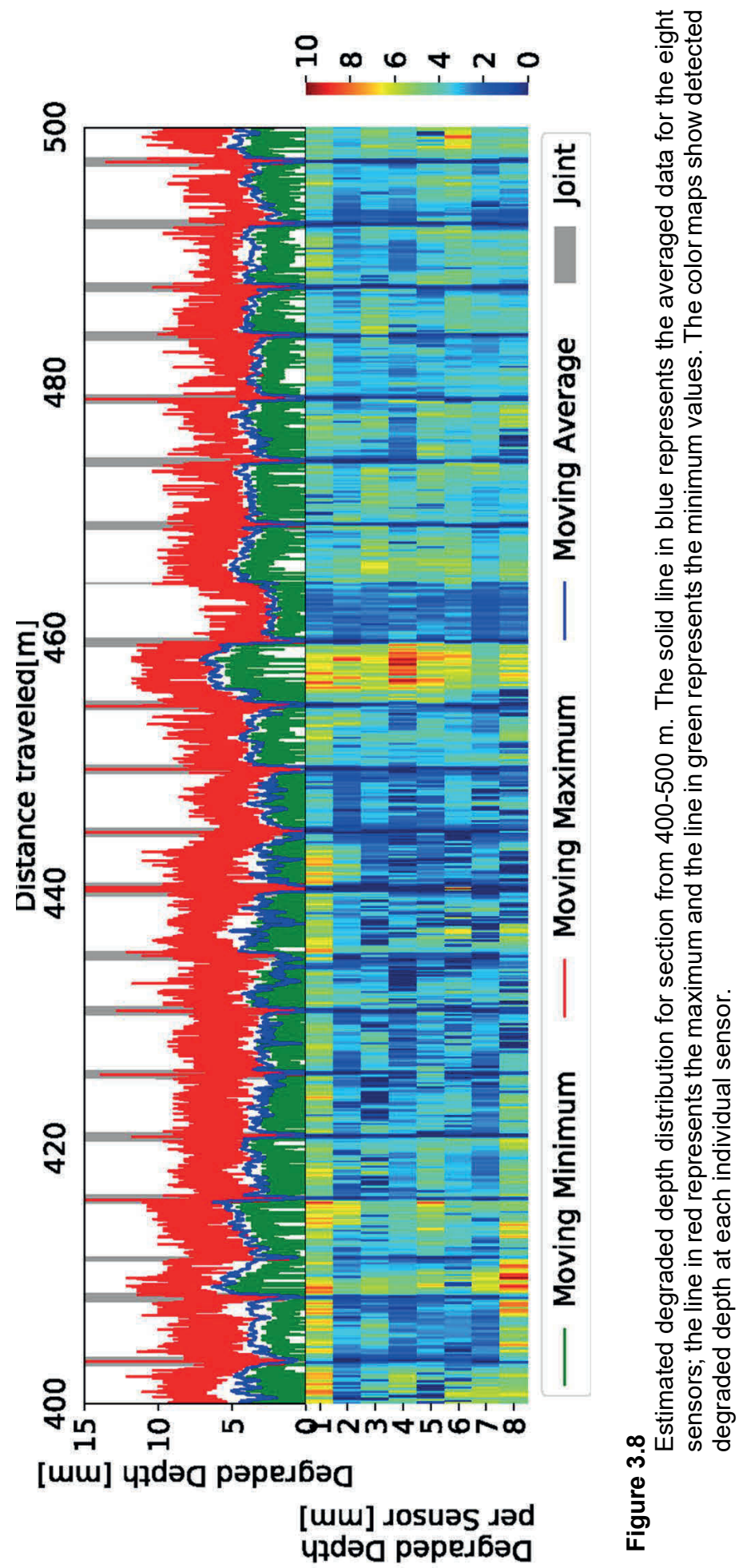


has low degradation depth. However, for the case at location $455 \mathrm{~m}$, the outlier is the pipe section with the highest degraded depth. A third possibility is that the assumption of constant pipe thickness is incorrect for those specific pipe sections. However, since no data on actual pipe thickness, or the thickness standard deviation as a result of the production process, is available, assuming a constant pipe thickness is the best option, especially considering that this assumption seems to hold for most pipe sections.

\section{(II) Gradual increase/decrease of degraded depth in a pipe section}

In Figure 3.9 at around $955 \mathrm{~m}$ and $985 \mathrm{~m}$ a considerable increase in degradation depth along one pipe section is observed. This would mean that the degradation progressively increases from the beginning of a single pipe segment towards the end. The difference between the highest and lowest degraded points is $7.34 \mathrm{~mm}$. This same behaviour is seen at $410 \mathrm{~m}$ in Figure 3.8. A possible explanation would be a variable wall thickness.

\section{(III) Consistent degradation along the longitudinal direction}

A third relevant behaviour that is observed throughout the inspected trajectory as a trajectory-wide behaviour. This effect can be better seen in Figure 3.10. The trend within each pipe section is not isolated (as in the previous examples) but it is consistent with the neighbouring pipe sections. For instance, from $1200 \mathrm{~m}$ to $1400 \mathrm{~m}$ in Figure 3.10 a higher degradation depth is seen compared to adjacent sections (from $1010 \mathrm{~m}$ to $1200 \mathrm{~m}$ and from 1400 to $1600 \mathrm{~m}$ ). This accounts for at least 200 meters of the inspected trajectory. Furthermore, the degradation depth changes gradually rather than sharply. A similar, but more localized, pattern can be seen in Figure 3.10, approximately at $50 \mathrm{~m}, 250 \mathrm{~m}, 750 \mathrm{~m}$ and $1000 \mathrm{~m}$. In these sections, there is high degradation depth compared to adjacent sections. Leaching of calcium is expected to occur more globally than locally, as it is caused by the surrounding environment, especially the soil. Thus, calcium leaching would be expected to be present more often in several adjacent pipes than in one pipe only.

Figure 3.10, the outliers found by the average per pipe and by the maximum per pipe are related to change in pipe material. An example of this can be better seen in Figure 3.9 in the travelled distance between $940 \mathrm{~m}$ and $950 \mathrm{~m}$ where the apparent degradation depth drops to zero. In that section of the network a different type of pipe was installed. Similarly, in the distances at approximately at $750 \mathrm{~m}$, $1500 \mathrm{~m}$ and $1770 \mathrm{~m}$, other types of pipes are detected (see Figure 3.10).

\section{(IV) Apparent difference in degradation along the circumference}

The fourth and last observed behaviour in the inspected trajectory is the apparent difference in degraded depth along the circumference of the pipe. Considering the assumed homogeneity in pipe wall thickness throughout the inspected trajectory, this would mean that degradation differs along the circumference in cement-based pipes. This effect is depicted in Figure 3.11 at $1660 \mathrm{~m}, 1610 \mathrm{~m}$ and $1645 \mathrm{~m}$, where 


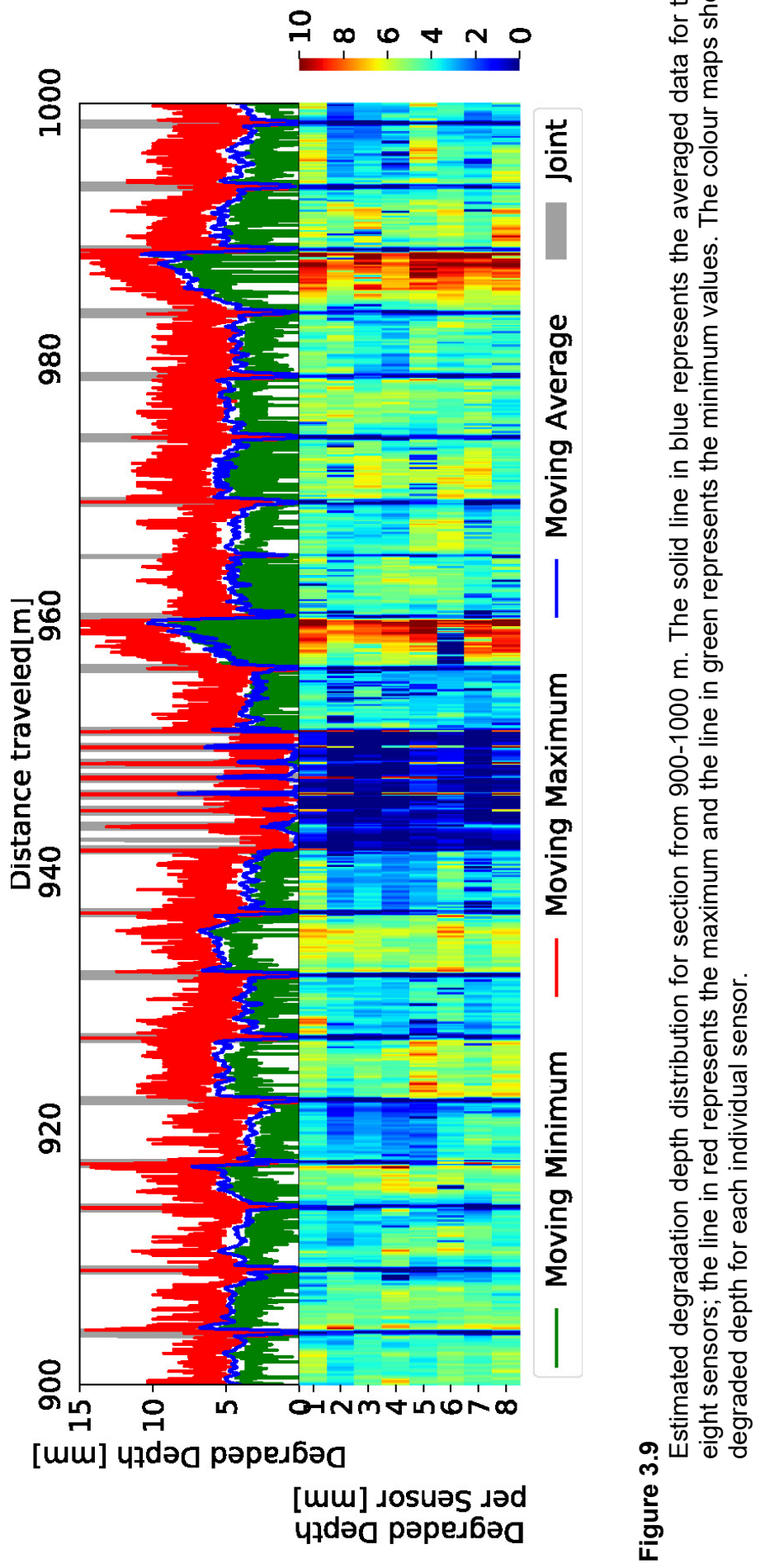



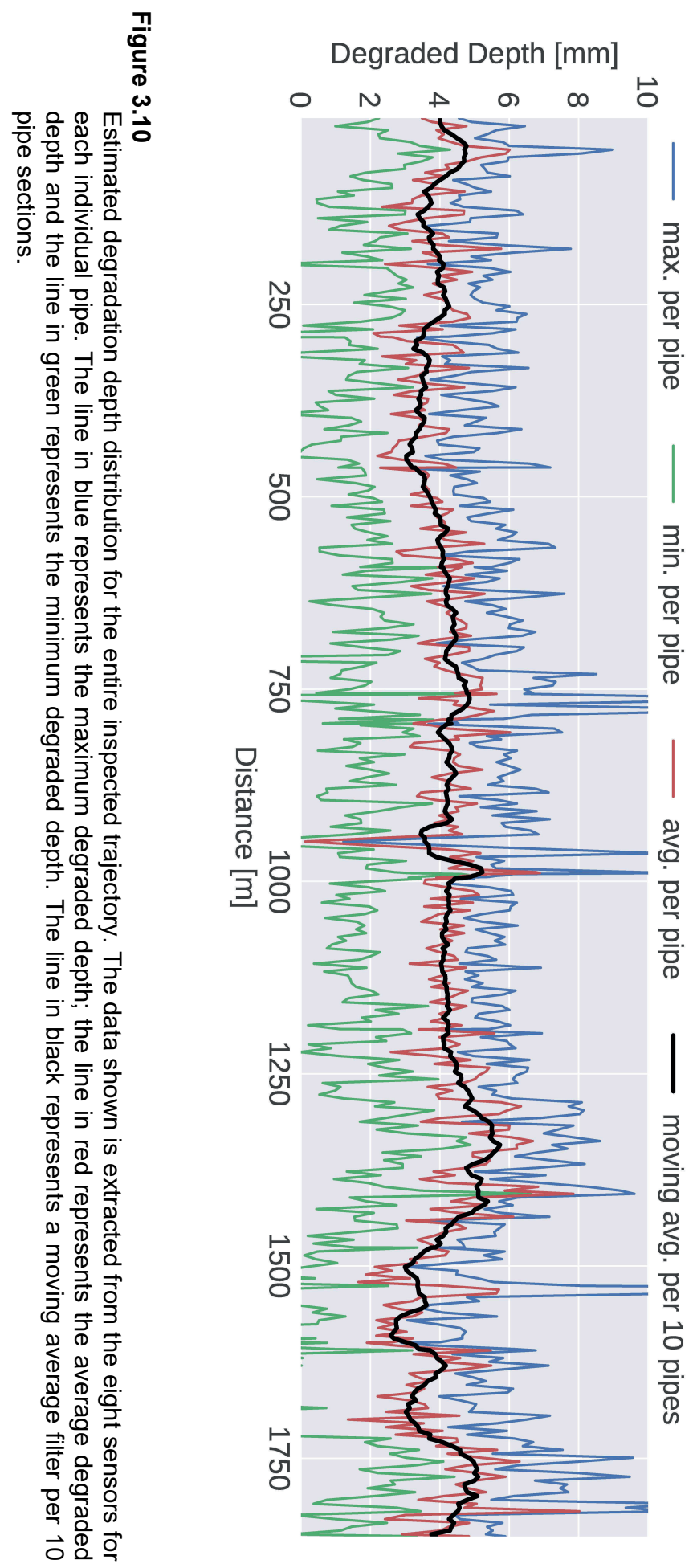





half of the sensors detect a higher degradation depth than the other half of the sensors. This behaviour is observed as well in Figure 3.9 at $920 \mathrm{~m}$.

The assumption that the thickness is constant throughout the inspected trajectory and along the circumference may not be true for all the pipe sections. However, in most of the inspected trajectory, the degraded depth is rather consistent along the circumference of the pipes.

After considering the four main types of deviations, the increase/decrease of estimated degraded depth in a single pipe section from start to end is the most remarkable behaviour. In the case shown in Figure 3.9 around $955 \mathrm{~m}$ and $985 \mathrm{~m}$, there is no correspondence at all with the adjacent pipes. Thus, the assumption that the thickness is constant is potentially wrong and the observed variation might be due to a manufacturing artefact. If this would be the case, the maximum apparent thickness change would be $6.25 \mathrm{~mm}$ (for a nominal thickness of $23 \mathrm{~mm}$ ). This thickness variation accounts for $25 \%$ change in thickness. Thus, it is likely to be more than just a manufacturing artefact, since even the lack of quality control from the pipe manufacturers 50 years ago cannot explain this large difference.

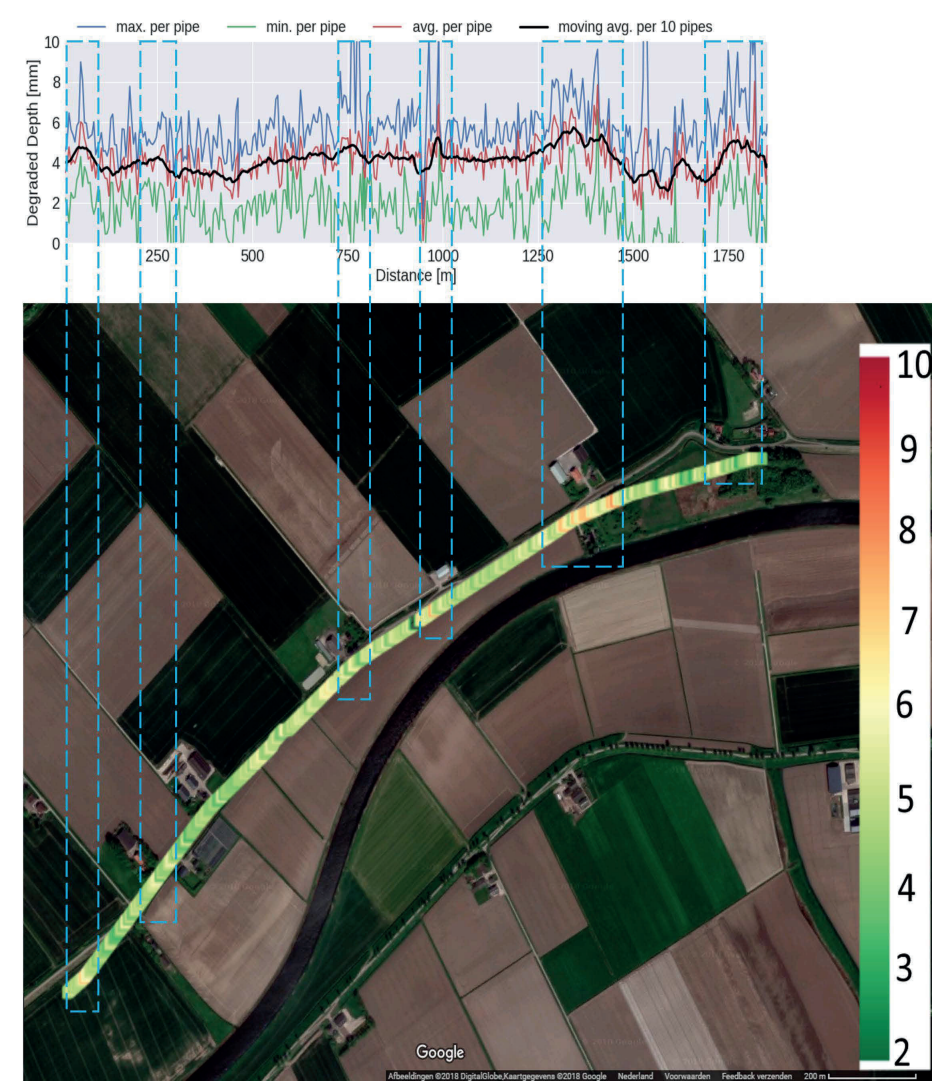

Figure 3.12

Aerial view of the estimated degraded depths along the inspected trajectory. 
In Figure 3.12, the calculated degraded depth variation is shown in an aerial view along the inspected trajectory. For this visualization, the average degradation depth from the eight sensors is used to display the degraded depth. However, with this visualization it is not possible to see when there is circumferential degradation. Thus, using the average degraded depth per eight sensors is not valid in cases with circumferential degradation. If the maximum and minimum degradation depths shown in Figure 3.11 increase or decrease together with the average degraded depth, then the estimated degraded depth at that location is not an outlier. When the maximum degraded depth increases and the average degraded depth does not change, the estimated degraded depth at that location is considered an outlier. The outliers are due to change in material pipe and due to low amplitude signals.

In correlating the areas with apparent higher degradation with the surrounding environment, an increase in degradation appears to be mostly in areas next to farms. The land usage can thus have a relevant effect on the degradation levels and on the degradation rate for instance a difference in soil pH or moisture. The degradation levels shown from the aerial view in the map is a very useful way to investigate the possible causes of changes in the ultrasonic responses. In this way a better understanding of the possible causes of degradation can lead to improved failure predictions.

\subsubsection{Sensitivity}

The parameters that could introduce deviations to the estimated depth are: the implemented filter parameter $\left(f_{c}\right)$, wall thickness, speed of sound in pristine state and speed of sound in fully degraded cement ( $\gamma$ in Equation 3.1). In this section, the sensitivity of each of these parameters is investigated. In Figure 3.13, the sensitivity to the estimation of the degraded depth is shown. The used ranges for the various parameters are shown in Table 3.1.

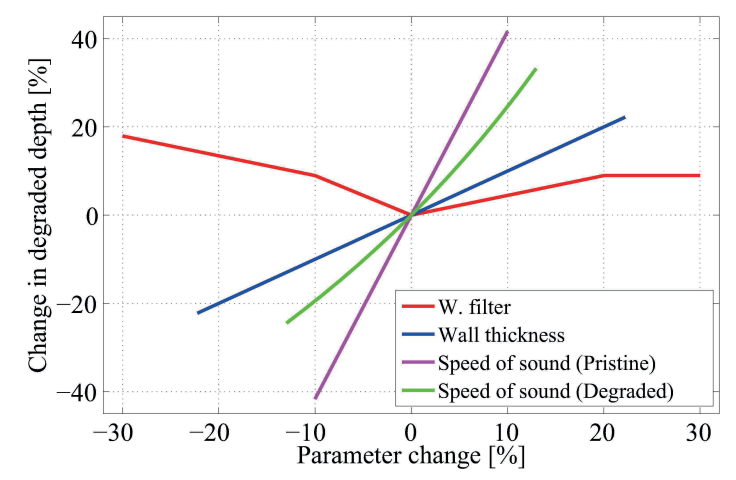

Figure 3.13

Sensitivity of the estimated degraded depth for variations in filter coefficient, pipe wall thickness, speed of sound in fully degraded material ( $\gamma$ in Equation 3.1) and speed of sound in pristine condition. 
Table 3.1 Upper and lower limits for the sensitivity analysis

\begin{tabular}{c|cccc}
\hline \multicolumn{1}{c}{} & $f_{C}$ & $D[\mathrm{~mm}]$ & $V_{h}[\mathrm{~m} / \mathrm{s}]$ & $\gamma$ \\
\cline { 2 - 5 } Lower limit & $\left(0.7 * f_{C}\right)$ & 18 & 4,500 & 0.47 \\
Upper limit & $\left(1.3 * f_{C}\right)$ & 28 & 5,500 & 0.61 \\
\hline
\end{tabular}

It is observed that the parameters with the highest sensitivity are the pristine and fully degraded speed of sound in cement, followed by the wall thickness. The filter parameters do not have a strong effect on the estimation of the degraded depth. A $10 \%$ deviation in the filter parameter induces only $3-7 \%$ error in the estimation of the degraded depth, depending on whether the parameter is increased or decreased.

A $5 \%$ change in the speed of sound in pristine condition already induces a $20 \%$ error in the estimation of the degraded depth. An error of $5 \%$ in the fully degraded speed of sound $\left(\gamma V_{h}\right)$ induces an error of $10 \%$ in the calculated degraded depth. Thus, these two parameters are fundamental. A $5 \%$ error in the wall thickness would induce a $5 \%$ error in the estimated degraded depth. Thus, there is a higher allowable range for errors in the thickness.

Assuming a constant thickness is an effective approach, however, in some isolated cases, this is not possible. The actual thickness of the installed infrastructure is unknown, thus determining the absolute value of the degraded depth remains a complex task. However, differences in apparent degradation depth can be clearly identified with the proposed method. These can then trigger further investigation with other techniques at only these specific locations. This selection of critical locations can already imply huge cost savings compared to a situation in which a complete section must be analysed in detail or even replaced.

\subsection{Conclusion}

In this research, the possibility to inspect and evaluate cement-based pipes with the ultrasonic pulse echo technique was confirmed. Furthermore, the developed method to process the ultrasonic responses can be used for a large amount of measurement data. The colour maps present a powerful tool to identify large changes in degraded depth within pipe sections in the inspected water grid as well as in the circumference of each pipe. Lastly, it also aids to recognize consistent degradation patterns. Averaging the degraded depth per eight sensors is a valid procedure only for identifying changes for large distances. For more detailed information on degradation levels, the use of colour maps is recommended.

It was found that the two most important parameters that have to be correctly determined are the speed of sound in the pristine condition and in the fully degraded condition. Along the inspected trajectory, the plot of the estimated degradation depth showed many interesting sections. Assuming that the pipe wall thickness is constant throughout the whole trajectory seems valid, since firstly the 
signals from the majority of the inspected trajectory were consistent with those of neighbouring pipes, and secondly since this assumption does not induce large errors in the calculated degraded depth.

\section{ACKNOWLEDGMENT}

This work was performed in the cooperation framework of Wetsus, European Centre of Excellence for Sustainable Water Technology (www.wetsus.eu). Wetsus is co-funded by the Dutch Ministry of Economic Affairs and Ministry of Infrastructure and Environment, the Province of Fryslân, and the Northern Netherlands Provinces. The authors like to thank the participants of the research theme "Smart Water Grids" for the fruitful discussions and their financial support. The authors thank Brabant Water and Acquaint B.V. for providing the inspection data. 


\section{REFERENCES}

1. P. J. J. Geudens and J. Grootveld, 'Dutch Drinking Water Statistics 2017,' 2017.

2. G. Stark and R. Charlton, 'Class 150 Asbestos Cement (AC) Watermain Pipe Condition Evaluation Deep Bay Water District,' vol. 106, no. December, pp. 3019-3032, 2008.

3. R. Chowdhury, Y. Hu, and D. Wang, 'Condition Evaluation of Asbestos Cement Water Mains,' in Pipelines 2012, 2012, no. 306, pp. 288-297.

4. V. Donazzolo and R. Yelf, 'Determination of wall thickness and condition of Asbestos Cement pipes in sewer rising mains using Surface Penetrating Radar,' Proc. 13th Internarional Conf. Gr. Penetrating Radar, GPR 2010, pp. 1-5, 2010.

5. J. Gong, M. Lambert, A. Zecchin, A. Simpson, N. Arbon, and Y. II Kim, 'Field study on non-invasive and non-destructive condition assessment for asbestos cement pipelines by time-domain fluid transient analysis,' Struct. Heal. Monit., vol. 15, no. 1, pp. 113-124, 2016.

6. D. H. Koo and S. T. Ariaratnam, 'Innovative method for assessment of underground sewer pipe condition,' Autom. Constr., vol. 15, no. 4, pp. 479488, 2006.

7. S. Smolders, L. Verhoest, G. De Gueldre, and B. van De Steene, 'Inspection of deteriorating asbestos cement force mains with georadar technique,' Water Sci. Technol., vol. 60, no. 4, pp. 995-1001, Apr. 2009.

8. G. Robbins, D. Johnston, and K. Laven, 'Predicting the Remaining Life of Asbestos Cement Pipe with Acoustic Wall Thickness Testing,' in Pipelines 2014, 2014, pp. 280-289.

9. M. Bracken, D. Johnston, and M. Coleman, 'Asset Management of Asbestos Cement Pipes Using Acoustic Methods: Theory and Case Studies,' in Pipelines 2011, 2011, pp. 225-235.

10. M. Bracken and D. Johnston, 'Acoustic Methods for Determining Remaining Pipe Wall Thickness in Asbestos Cement and Ferrous Pipes,' in Pipelines 2009, 2009, pp. 271-281.

11. Y. Hu, D. Wang, and R. Chowdhury, 'Condition Assessment Methods for AC Pipe and Current Practices,' in Pipelines 2010, 2010, no. 306, pp. 867-877.

12. H. Hernandez Delgadillo, B. Kern, R. Loendersloot, D. Yntema, and R. Akkerman, 'A Methodology Based on Pulse-Velocity Measurements to Quantify the Chemical Degradation Levels in Thin Mortar Specimens,' J. Nondestruct. Eval., vol. 37, no. 4, pp. 1-13, 2018.

13. H. H. Delgadillo, R. Loendersloot, R. Akkerman, and D. Yntema, 'Development of an inline water mains inspection tech nology,' in 2016 IEEE International Ultrasonics Symposium (IUS), 2016, vol. 2016-Novem, pp. 1-4.

14. D. Wang and D. R. Cullimore, 'Bacteriological challenges to asbestos cement water distribution pipelines,' J. Environ. Sci., vol. 22, no. 8, pp. 1203-1208, 2010.

15. D. Wang, R. Cullimore, Y. Hu, and R. Chowdhury, 'Biodeterioration of asbestos cement (AC) pipe in drinking water distribution systems,' Int. Biodeterior. Biodegrad., vol. 65, no. 6, pp. 810-817, 2011. 
16. F. Bouchaala, C. Payan, V. Garnier, and J. P. Balayssac, 'Carbonation assessment in concrete by nonlinear ultrasound,' Cem. Concr. Res., vol. 41, no. 5, pp. 557-559, 2011.

17. C. Carde and R. François, 'Effect of the leaching of calcium hydroxide from cement paste on mechanical and physical properties,' Cem. Concr. Res., vol. 27, no. 4, pp. 539-550, 1997.

18. D. Wang, Y. Hu, and R. Chowdhury, 'Examination of Asbestos Cement Pipe Deterioration with Scanning Electron Microscopy,' in Pipelines 2011, 2011, no. 306, pp. $65-78$.

19. N. Burlion, D. Bernard, and D. Chen, 'X-ray microtomography: Application to microstructure analysis of a cementitious material during leaching process,' Cem. Concr. Res., vol. 36, no. 2, pp. 346-357, 2006. 


\title{
Chapter 4
}

\section{Steering the propagation direction of a non-linear acoustic wave in a solid material}

\begin{abstract}
Non-collinear wave mixing is used as a non-destructive testing method where the amplitude of the scattering wave contains information on the condition of a material. The practical implementation of non-collinear wave mixing as a non-destructive testing technique is limited by many factors such as the geometry and shape of the structure, the accessibility to the specimen's surfaces and the ultrasonic sensors available to perform measurements. A novel approach to steer the propagation direction of a generated wave from the mixing of two incident acoustic waves is proposed. The angle of the scattering wave is controlled by the frequencies of the two interaction waves, rather than by the angle between these waves. The scattering amplitude was analytically solved for the longitudinal plus shear interaction process. The analytical solution was validated with experiments. The model qualitatively agrees with the experiments. Furthermore, the possibility to use a wider range of excitation frequencies of the incident waves was found. This is a great advantage in applications where the space and access to the specimen under test is limited.
\end{abstract}

This chapter has been published as: H. Hernandez Delgadillo, R. Loendersloot, D. Yntema, T. Tinga, and R. Akkerman, "Steering the propagation direction of a non-linear acoustic wave in a solid material," Ultrasonics, vol. 98, no. April, pp. 28-34, 2019. 


\subsection{Introduction}

In recent years, the generation of an acoustic wave from the mixing of two incident waves has been of interest for researchers because of its great advantages. Applications such as detection of plasticity in metals, detection of micro-cracks, fatigue and detection of physical ageing in plastics make it very attractive [1-7]. Furthermore, the detection of imperfect interfaces with the wave mixing technique has been a subject of research as well [8-12]. For instance, the measured amplitude of the generated wave was found to be directly related to the acoustic parameter $\beta$. Direct correlation was found between this parameter and damage in solids such as low adhesive joint quality, weathering damage in limestone blocks and plastic deformation in aluminium [3, 13-15]. The measured amplitude of the generated wave has a relatively high sensitivity to any of the changes in the conditions mentioned above compared to linear ultrasonics. In recent work, Demčenko demonstrated that with two-sided non-collinear wave mixing configuration it is possible to detect ageing in grey polyvinyl chloride (PVC) with good sensitivity compared to linear ultrasonics in the longitudinal wave mode [5].

The scattering field of a wave generated from the local resonance of two incident waves in a solid medium was derived in 1968 from the linear theory of elasticity and with the time-dependent perturbation theory [16-19]. In a more recent research, Korneev et al. [20] presented a corrected version of the derivation done in 1968, as well as the solution of an amplitude coefficient for the ten possible interaction processes. The solutions of these are aimed to be used to find the optimal testing parameters.

In the available literature, the propagation direction is calculated from the input frequencies of the incident waves. In none of the work found, the direction of the generated wave is steered. This means that no experimental set-up has been designed such that it has the accuracy to change the angles and distances between sensors and the testing material. The possibility to mechanically steer the generated wave becomes challenging in applications limited by the space, weight and energy available. When access to one surface only is possible, the complexity of a testing system further increases. It is commonly found that for laboratory set-ups, the access to more than one surface is attainable. However, positioning of the sensors in many cases for real testing of structures has limitations. For instance, the inspection of PVC pipes requires access to only the internal or external surface. Thus, in order to upscale a testing configuration towards NDE purpose, the settings have to be optimized.

The objective of this research is the optimization of a wave mixing configuration. This is done by demonstrating from experiments the possibility to steer the propagation direction of a wave generated from the mix of two incident waves by changing the incident wave's excitation frequencies only. The latter is achieved while keeping the two incident angles constant. This considerably simplifies the testing conditions, as the two sensors only need to be positioned once. Thereafter, only the receiver has to be adjusted according to the propagation direction of the 
generated wave. Furthermore, by having a constant interaction angle and a variable excitation frequency, the optimal settings can be directly adjusted by changing the pump wave's frequencies when the receiver's location cannot be adjusted.

The outline of this paper is as follows. In section 4.2 , the wave mixing theory will be shortly discussed. In section 4.3, the methodology is explained. First, in the analytical part, the assumptions to the solution of the equations shown in section 4.2 are explained. In the second part of section 4.3 , the experimental campaign used to validate the analytical solution is described in detail. Section 4.4 then presents and discusses all the results of both the analytical and experimental work, and finally section 4.5 forwards the main conclusions.

The main contributions of this research are: $(i)$ providing the solution of the scattering amplitude and the interaction volume for this interaction process as a function of the second pump wave frequency at a constant interaction angle $\alpha$; (ii) presenting the idea to steer the direction of the generated wave by only changing the frequencies of the incident waves, based on this solution. Thus, significantly reducing the complexity of a test set-up and still allowing optimization of the generated wave.

\subsection{Wave mixing theory}

The local resonance of two incident acoustic waves generates a third acoustic wave which propagates at an angle $\psi$ with respect to one of the incident waves. In Figure 4.1 this phenomenon is depicted where the incident wave vectors are $\mathbf{k}_{1}$ and $\mathbf{k}_{2}$ and $\mathbf{k}_{g}$ is the generated wave vector.

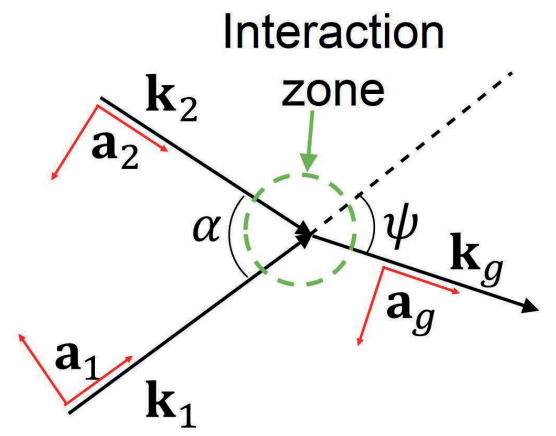

Figure 4.1

Wave $\mathbf{k}_{g}$ generated from the local resonance of two incident acoustic waves ( $\mathbf{k}_{1}$ and $\mathbf{k}_{2}$ ). The symbol $\mathbf{k}_{i}$ indicates the wave vectors and $\mathbf{a}_{i}$ the polarization of the waves.

The interaction of these two waves is defined by the cosine law

$$
\left(\mathbf{k}_{1}\right)^{2}+\left(\mathbf{k}_{2}\right)^{2} \pm 2 \overline{\mathbf{k}}_{1} \overline{\mathbf{k}}_{2} \cos \alpha=\left(\mathbf{k}_{g}\right)^{2}
$$


where $\alpha$ is the interaction angle between $k_{1}$ and $k_{2}$ and the magnitude of the wave vectors is

$$
\overline{\mathbf{k}}_{n}=\frac{\omega_{n}}{\overline{\mathbf{v}}_{n}}
$$

where $\omega_{n}$ is the frequency of the incident waves (1 and 2) and the generated wave (g), $\overline{\mathbf{v}}_{n}$ is either the longitudinal or shear speed of sound in the material. The generated wave vector propagates under an angle with respect to $\mathbf{k}_{1}$ equal to

$$
\psi=\tan ^{-1}\left(\frac{ \pm \frac{v_{1}}{v_{2}} d \sin \alpha}{1 \pm \frac{v_{1}}{v_{2}} d \cos \alpha}\right)
$$

where $d=\omega_{2} / \omega_{1}$. This is depicted in Figure 4.1.

The resonant conditions at which this process occurs are

$$
\begin{aligned}
& \mathbf{k}_{g}=\mathbf{k}_{1} \pm \mathbf{k}_{2} \\
& \omega_{g}=\omega_{1} \pm \omega_{2}
\end{aligned}
$$

The local resonance of two incident waves cannot be described with the linear elastic theory if the equation of motion is linear due to its nonlinear nature $[18,20]$. The equation of motion in the Cartesian form is

$$
\rho \frac{\partial^{2} \mathbf{u}}{\partial t^{2}}-\mu \nabla^{2} \mathbf{u}-(\lambda+\mu) \nabla(\nabla \cdot \mathbf{u})=\mathbf{F}
$$

where $\rho$ is the density of the material, $\mu$ and $\lambda$ are the Lamé parameters, $\mathbf{F}$ is an external force, $\mathbf{u}$ the particle displacement vector. In order to have a non-linear equation of motion which considers the interaction between two plane waves, cubic terms are included in the particle displacements [18, 20].

The sum of the two incident waves is assumed to be

$$
\mathbf{u}_{0}=A_{1} \cos \left(\omega_{1} t-\left(\mathbf{k}_{1} \cdot \mathbf{r}\right)\right) \mathbf{a}_{1}+A_{2} \cos \left(\omega_{2} t-\left(\mathbf{k}_{2} \cdot \mathbf{r}\right)\right) \mathbf{a}_{2}
$$

where $\mathbf{r}$ is the vector from the center of interaction to the observation point (position of receiver) and $\mathbf{a}_{1}$ and $\mathbf{a}_{2}$ are the polarization vectors of the incident waves. The polarization vector is parallel to the propagation direction for longitudinal waves (see Figure 4.1), and perpendicular to the propagation direction for shear waves as shown in Figure 4.1. The following is obtained from substituting Equation 4.7 in Equation 4.6

$$
\begin{aligned}
\mathbf{p}(\mathbf{r}, t)=-A_{1} A_{2} & \left(\mathbf{I}^{+} \sin \left[\left(\omega_{1}+\omega_{2}\right) t-\left(\mathbf{k}_{1}+\mathbf{k}_{2}\right) \mathbf{r}\right]\right. \\
& \left.+\mathbf{I}^{-} \sin \left[\left(\omega_{1}-\omega_{2}\right) t-\left(\mathbf{k}_{1}-\mathbf{k}_{2}\right) \mathbf{r}\right]\right)
\end{aligned}
$$


where $A_{1}$ and $A_{2}$ are the amplitudes of the incident waves. The \pm refers to the interaction process and it can be either the sum or the difference. In Equation 4.8, the $\mathbf{p}$ vector is a component of the force $\mathbf{F}$ that contains the interactions of the acoustic waves $[18,20]$. This vector is a time dependent function and a function of the observation point (measurement location). The vector $\mathbf{I}^{ \pm}$is defined as

$$
\begin{aligned}
\mathbf{I}^{ \pm}=\frac{1}{2} & C_{1}\left[\left(\mathbf{a}_{1} \cdot \mathbf{a}_{2}\right)\left(k_{2}^{2} \mathbf{k}_{1} \pm k_{1}^{2} \mathbf{k}_{2}\right)+\left(\mathbf{a}_{2} \cdot \mathbf{k}_{1}\right) k_{2}\left(k_{2} \pm 2 k_{1} \cos \alpha\right) \mathbf{a}_{1}\right. \\
& \left.+\left(\mathbf{a}_{1} \cdot \mathbf{k}_{2}\right) k_{1}\left(2 k_{2} \cos \alpha \pm k_{1}\right) \mathbf{a}_{2}\right] \\
& +\frac{1}{2} C_{2} k_{1} k_{2} \cos \alpha\left[\left(\mathbf{a}_{1} \cdot \mathbf{a}_{2}\right)\left(\mathbf{k}_{2} \pm \mathbf{k}_{1}\right)+\left(\mathbf{a}_{2} \cdot \mathbf{k}_{2}\right) \mathbf{a}_{1} \pm\left(\mathbf{a}_{1} \cdot \mathbf{k}_{1}\right) \mathbf{a}_{1}\right] \\
& +\frac{1}{2} C_{3}\left[\left(\mathbf{a}_{1} \cdot \mathbf{k}_{2}\right)\left(\left(\mathbf{a}_{2} \cdot \mathbf{k}_{2}\right) \pm\left(\mathbf{a}_{2} \cdot \mathbf{k}_{1}\right)\right) k_{1}\right. \\
& \left.+\left(\mathbf{a}_{2} \cdot \mathbf{k}_{1}\right)\left(\left(\mathbf{a}_{1} \cdot \mathbf{k}_{2}\right) \pm\left(\mathbf{a}_{1} \cdot \mathbf{k}_{1}\right)\right) k_{2}\right] \\
& +\frac{1}{2} C_{4}\left(\mathbf{a}_{2} \cdot \mathbf{k}_{2}\right)\left[\left(\mathbf{a}_{1} \cdot \mathbf{k}_{2}\right) \mathbf{k}_{2} \pm\left(\mathbf{a}_{1} \cdot \mathbf{k}_{1}\right) \mathbf{k}_{1}\right] \\
& +\frac{1}{2} C_{5}\left[\left(\mathbf{a}_{1} \cdot \mathbf{k}_{1}\right) \boldsymbol{k}_{2}^{2} \mathbf{a}_{2} \pm\left(\mathbf{a}_{2} \cdot \mathbf{k}_{2}\right) k_{1}^{2} \mathbf{a}_{1}\right]
\end{aligned}
$$

where $C_{1}$ to $C_{5}$ are functions of the third order elastic constant (TOEC) and the Lamé parameters $[18,20]$. In the vector $\mathbf{I}^{ \pm}$all the possible interactions are included. Thus, when selecting a specific interaction process, and depending on the type of wave (longitudinal or shear), the dot products within the vector will take a value of either one or zero. It is key to understand that the vector $\mathbf{I}^{ \pm}$contains the information of the material properties that change due to damage, which cannot be detected by linear ultrasonics. For example, the information contained in the vector $\mathbf{I}^{ \pm}$for the horizontal shear plus horizontal shear interaction process is captured by $C_{1}$ and $C_{2}$ only. The constants are defined as follow

$$
\begin{aligned}
C_{1} & =\mu+\frac{n}{4}, & C_{2} & =\lambda+\mu+m-\frac{n}{4},
\end{aligned}
$$

where $l, m$ and $n$ are the third order elastic constants. The complete derivation of the scattering field can be found in $[18,20]$, and yields

$$
\mathbf{u}(\mathbf{r}, t)=\frac{A_{1} A_{2}}{4 \pi r \rho} \sum_{\xi=+,-}\left(\frac{\left(\mathbf{I}^{\xi} \cdot \hat{\mathbf{r}}\right) \hat{\mathbf{r}}}{v_{L}^{2}} V_{L}^{\xi}+\frac{\mathbf{I}^{\xi}-\left(\mathbf{I}^{\xi} \cdot \hat{\mathbf{r}}\right)}{v_{s}^{2}} V_{s}^{\xi}\right)
$$

where $\hat{\mathbf{r}}$ is the unit vector of the observation point (measurement point), $v_{L}$ and $v_{S}$ are the longitudinal and shear speed of sound respectively and $V_{L}$ and $V_{S}$ are the interaction volumes if the generated wave is longitudinal or shear respectively. This solution entails all the possible interaction processes as it contains the vector $\mathbf{I}^{ \pm}$. 


\subsection{Methodology}

In order to demonstrate that the generated wave can be steered other than by mechanically adjusting the interaction angles, the amplitude component from Equation 4.11 was analytically solved for the longitudinal + shear (SV) interaction process. Furthermore, a set of experiments were performed in order to validate the solution for the amplitude of the generated wave as a function of the excitation frequencies.

\subsubsection{Analytical solution}

The scattering field from Equation 4.11 for the longitudinal plus shear interaction process that generates a longitudinal wave is

$$
\mathbf{u}(\mathbf{r}, t)=\frac{A_{1} A_{2}}{4 \pi r \rho} \frac{\left(\mathbf{I}^{+} \cdot \hat{\mathbf{r}}\right) \hat{\mathbf{r}}}{v_{L}^{2}} V_{L}^{+}
$$

The zone where the two incident beams interact is dependent on the wave length, speed of sound, amplitude and propagation direction of both beams (see Figure 4.2). This zone is called the interaction volume. The given Equation is from [18]. The interaction volume for a generated longitudinal wave is defined as

$$
V_{L}^{+}=\int \sin \left(\left(\omega_{1}+\omega_{2}\right)\left(\frac{\mathrm{r}}{v_{g}}-t\right)-\left(\mathbf{k}_{1}+\mathbf{k}_{2}-\frac{\omega_{1}+\omega_{2}}{v_{g}} \hat{\mathbf{r}}\right) r^{\prime}\right) d V
$$

Furthermore, the vector $\mathbf{I}^{ \pm}$for this interaction process is reduced to

$$
\begin{aligned}
\mathbf{I}_{l+s}=\frac{1}{2} C_{1}\left[\left(\mathbf{a}_{1} \cdot\right.\right. & \left.\mathbf{a}_{2}\right)\left(k_{2}^{2} \mathbf{k}_{1}+k_{1}^{2} \mathbf{k}_{2}\right)+\left(\mathbf{a}_{2} \cdot \mathbf{k}_{1}\right) k_{2}\left(k_{2}+2 k_{1} \cos \alpha\right) \mathbf{a}_{1} \\
& \left.+\left(\mathbf{a}_{1} \cdot \mathbf{k}_{2}\right) k_{1}\left(2 k_{2} \cos \alpha+k_{1}\right) \mathbf{a}_{2}\right] \\
& +\frac{1}{2} C_{2} k_{1} k_{2} \cos \alpha\left[\left(\mathbf{a}_{1} \cdot \mathbf{a}_{2}\right)\left(\mathbf{k}_{2}+\mathbf{k}_{1}\right)+1\right] \\
& +\frac{1}{2} C_{3}\left[\left(\mathbf{a}_{1} \cdot \mathbf{k}_{2}\right)\left(\left(\mathbf{a}_{2} \cdot \mathbf{k}_{1}\right)\right) k_{1}\right. \\
& \left.+\left(\mathbf{a}_{2} \cdot \mathbf{k}_{1}\right)\left(\left(\mathbf{a}_{1} \cdot \mathbf{k}_{2}\right)+1\right) k_{2}\right]+\frac{1}{2} C_{5}
\end{aligned}
$$

In this case only the $C_{4}$ component is zero. The general solution of the scattering amplitude depends on the integral of the interaction volume as shown in Equation 4.13. If the volume of interaction is assumed to be a sphere, the amplitude of the scattering wave is

$$
\mathrm{A}=\frac{R^{3}}{3} \frac{\left(\mathbf{I}_{l+s}^{+} \cdot \hat{\mathbf{r}}\right)}{v_{l}^{2} \rho} \frac{A_{1} A_{2}}{r}
$$


where $R$ is the radius of the sphere. The radius is calculated by taking into account the diameter of the transducers and their beam divergence. Thus, the radius of the sphere is proposed to be defined as

$$
R=0.5 \sqrt{a^{2}+\left(D_{s 2}+\left(a \tan \gamma_{s 2}\right)\right)^{2}}
$$

where $D_{s 2}$ is the diameter of the piezoelectric element of sensor two; $a$ is the distance that fits the width of beam path one to the length of beam path two; $b_{p}$ is the relative distance with respect to $a$ as a function of the beam divergence $\gamma_{s 2}$ of beam two. The beam divergence is calculated from the $6 \mathrm{~dB}$ decrease from the central beam path. Thus, the change in $f_{1}$ and $f_{2}$ are considered in terms $a$ and $\gamma_{s 2}$ respectively in Equation 4.16. The interaction of the beam patterns is shown in Figure 4.2. The beam paths are approximated as trapezoids. The proposed calculation of the interaction volume as a function of the pump wave frequencies is not available in the literature, and can thus be considered as a contribution of this research.
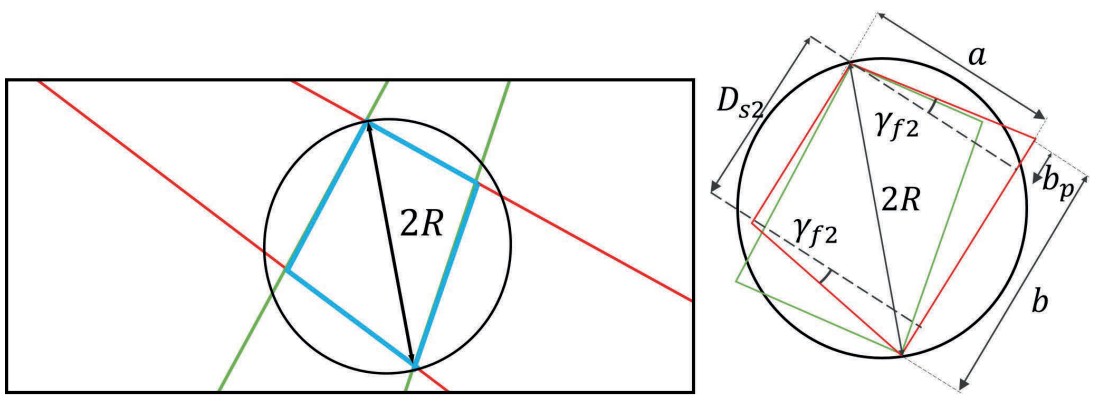

Figure 4.2

Beam pattern of refracted waves inside the material showing how the beams of the refracted waves fit in the interaction zone. Trapezoid in red is the beam path of the refracted shear wave, the trapezoid in green is the beam path of the refracted longitudinal.

Values for the constants in Equations 4.10, in this case for PVC are obtained from the literature [20]: $\lambda=3.64 \mathrm{GPa}, \mu=1.83 \mathrm{GPa}, l=-33.43 \mathrm{GPa}, m=-20.88$ GPa, $n=-15.86 \mathrm{GPa}$ and $\rho=1350 \mathrm{~kg} / \mathrm{m}^{3}$.

\subsubsection{Experimental set-up}

The interaction process is $L_{1}+S_{2}=L$. The incident angle of the pump wave one $f_{1}$ was selected such that the refracted longitudinal wave is below the first critical angle. The amplitude of the refracted shear wave component before the first critical angle is much smaller than to the amplitude of the longitudinal wave (see Figure 4.3b). The incident angle of the pump wave two $f_{2}$ was selected above the first critical angle (see Figure 4.3a). The maximum amplitude of a shear wave 
component is achieved with an incident angle of approximately $50^{\circ}$ (see Figure 4.3b). Figure 4.3 thus depicts the possible incident angles that will generate the refracted longitudinal and shear waves inside the PVC material. For these experiments, the refracted longitudinal angle is $\theta_{r l}=67^{\circ}$ and the refracted shear angle is $\theta_{r s}=31^{\circ}$. The interaction angle is $\alpha=98^{\circ}$.

(a)

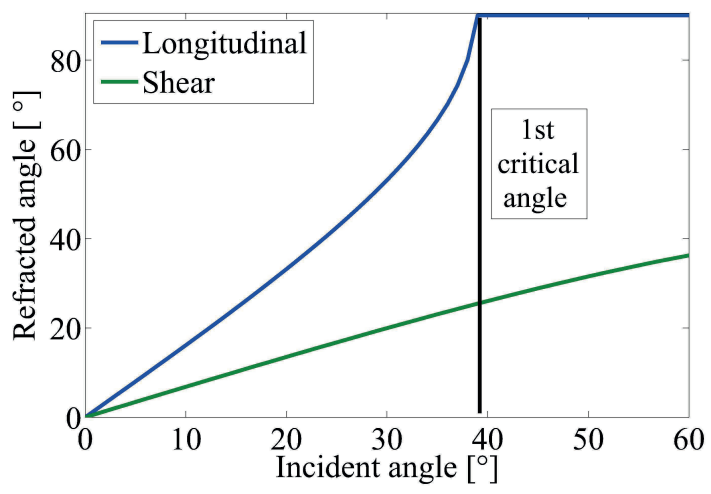

(b)

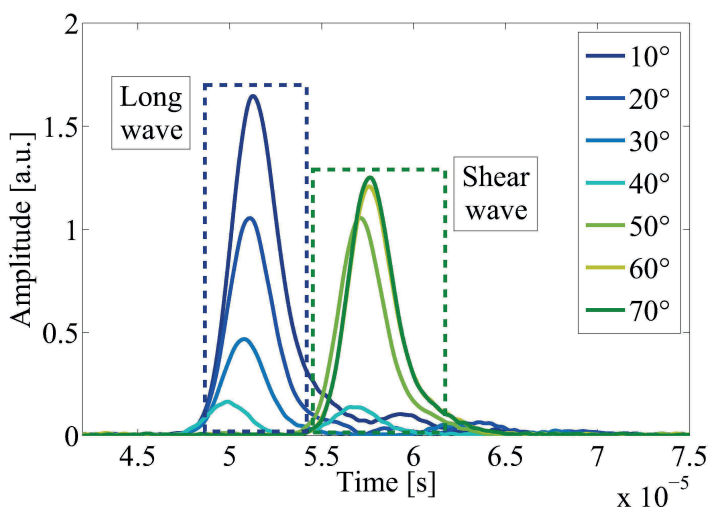

Figure 4.3

Longitudinal and shear (a) refracted angles for water-PVC interface and (b) refracted longitudinal and shear wave amplitudes at different incident angles. The envelope of the measured signals is shown only.

A sinusoidal input voltage with 30 cycles (lowest frequency) and 40 cycles (highest frequency) were used for the pump waves. Despite that the bursts do not completely fit at the same time in the interaction zone, increasing the number of cycles improves the resolution of the generated wave in the frequency domain. No further interaction occurs after the waves travel beyond the interaction zone. A longer interaction is not found to be necessary. The excitation frequency of the first incident wave was kept constant while the excitation frequency of the second incident wave was gradually increased as shown in Table 4.1. 
Table 4.1 Input frequencies, frequency ratio $d=\omega_{2} / \omega_{1}$ and generated frequency for the interaction process.

\begin{tabular}{|cccc|}
\hline$d$ & $\begin{array}{c}f_{1} \\
(\mathrm{kHz})\end{array}$ & $\begin{array}{c}f_{2} \\
(\mathrm{kHz})\end{array}$ & $\begin{array}{c}f_{g} \\
(\mathrm{kHz})\end{array}$ \\
1.3 & 650 & 850 & 1500 \\
1.384 & 650 & 900 & 1550 \\
1.461 & 650 & 950 & 1600 \\
1.538 & 650 & 1000 & 1650 \\
1.615 & 650 & 1050 & 1700 \\
1.692 & 650 & 1100 & 1750 \\
1.769 & 650 & 1150 & 1800 \\
1.846 & 650 & 1200 & 1850 \\
1.923 & 650 & 1250 & 1900 \\
2 & 650 & 1300 & 1950 \\
2.076 & 650 & 1350 & 2000 \\
\hline
\end{tabular}

Three ultrasonic transducers were placed according to the selected incident angles. The set-up is shown in Figure 4.4, where the dimensions of the PVC samples are $l=150 \mathrm{~mm}, w=35 \mathrm{~mm}$ and thickness $t=15 \mathrm{~mm}$. The sensors used were two flat $1 \mathrm{MHz}$ central frequency transducers and the receiver used was a flat broad-band $2.25 \mathrm{MHz}$ central frequency transducer. The central frequency of these transducers is standard and is close to the frequencies shown in Table 4.1.

The interaction depth is calculated based on the initial positioning of the sensors. The initial position of the sensors is taken from literature where a longitudinal plus shear wave interaction was investigated in a PVC specimen [5]. The travel path of each wave is calculated as

$$
\begin{aligned}
& l_{\text {path }}=\frac{x_{m} \sin \left(90-\theta_{r s}\right)}{\sin \alpha} \\
& s_{\text {path }}=\frac{x_{m} \sin \left(90-\theta_{r l}\right)}{\sin \alpha}
\end{aligned}
$$


where $x_{m}$ is the distance between the two beams refract in the material. The interaction depth of these acoustic waves is calculated as

$$
h=l_{\text {path }} \cos \theta_{r l}=s_{\text {path }} \cos \theta_{r s}
$$

The set-up including the electronic equipment is shown in Figure 4.5. Before amplification, the voltage of the signal generator is $80 \mathrm{mV}$. The peak to peak voltage sent to the transducers is $90 \mathrm{~V}$. The signal generator has two independent channels. These are synchronized by a trigger. The details on the manufacturers of the equipment used for this research are shown in Table 4.2.
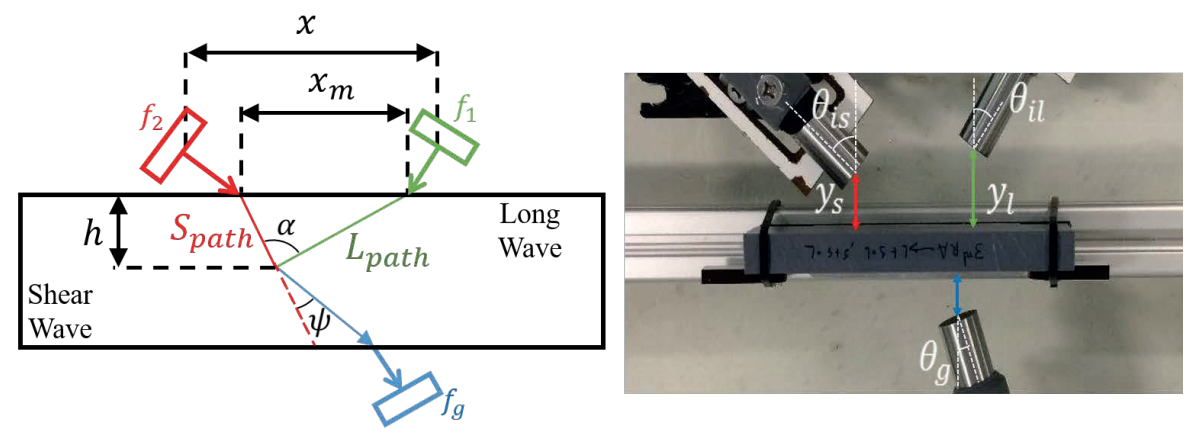

Figure 4.4

Sensor positioning for longitudinal + shear interaction process. Interaction depth and the travel path of each wave component is depicted.

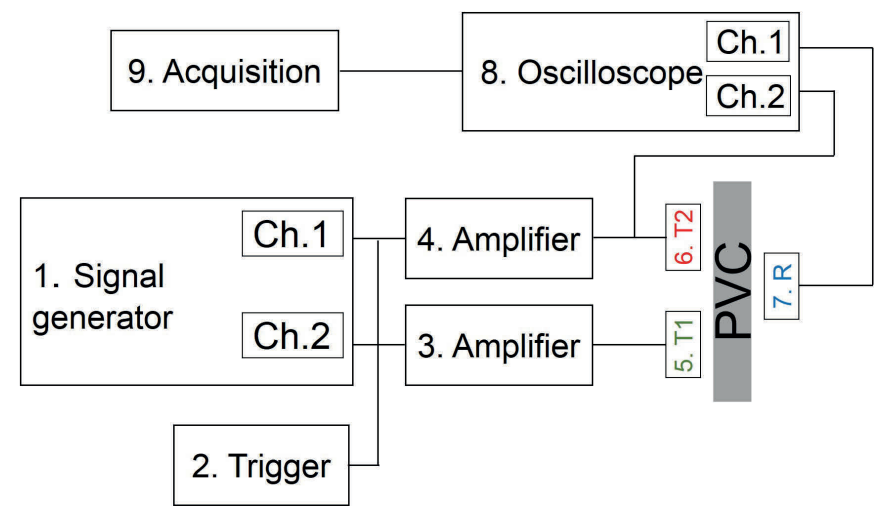

Figure 4.5

Set-up including the electronic equipment.

The amplitude of the generated wave was recorded with a 15-bit resolution and $125 \mathrm{MS} / \mathrm{s}$ acquisition rate. During the experiments, a time-delay was applied to the second incident wave in order to compensate for the time-of-flight change due to the change in frequency due to the material dispersion [5]. The time-delay was adjusted until the maximum amplitude of the generated wave was found. From 
each experiment, the maximum peak of generated wave component in the frequency spectrum was extracted for 32 signals, averaged and then the standard deviation was calculated. The experimental set-up was disassembled and assembled for a second set of experiments.

Table 4.2 Set-up components

\begin{tabular}{ccc}
\hline 1. Signal generator & 2. Trigger & 3. Amplifier \\
\hline Keysight 33512B & Aim-TTi TG 2000 & Tomco BTM00250 \\
\hline 4. Amplifier & 5. Transmitter 1 & 6. Transmitter 2 \\
\hline Tomco BTM00250 & Sofranel IBHG014 & Olympus 14-0110 \\
\hline 7. Receiver & 8. Oscilloscope & 9. Acquisition \\
\hline Olympus 14-0210 & Picoscope 5442B & Computer \\
\hline
\end{tabular}

The complete time-domain signals were transferred to the frequency domain as shown in Figure 4.6. Three components are identified and the maximum amplitude of the generated wave frequency component is extracted as shown in Figure 4.6.

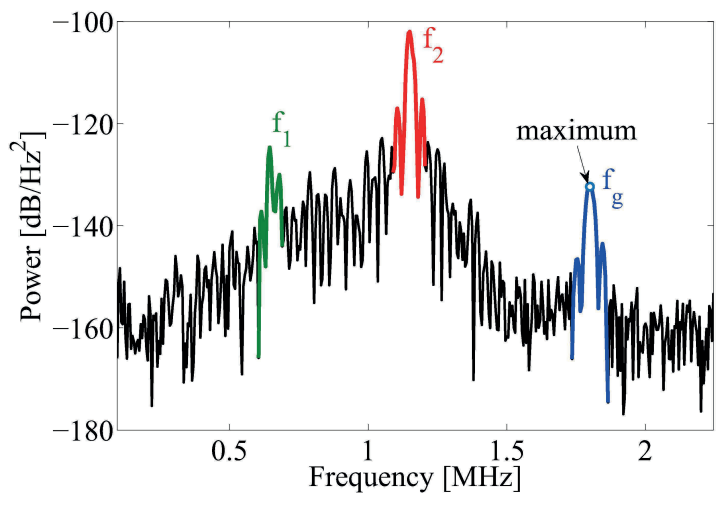

Figure 4.6

Frequency spectrum of a typical signal from the wave mixing testing. The circle in blue is the maximum amplitude of the generated wave frequency component.

The amplitude extracted from the frequency spectrum of the generated wave was corrected with the transfer function of the transducers. The data sheet from the 
transducer's manufacturer was used to derive a correction function. In this manner, the energy of the acoustic wave was compensated as the sensors were excited at frequencies other than the central frequency. Then the amplitude in the frequency spectrum was compensated as

$$
A=\frac{A_{m}}{S_{T 2} S_{R}}
$$

where $A_{m}$ is the amplitude of the generated wave in the frequency spectrum and $S_{T 2}, S_{R}$ are the transfer functions of the transmitter two and the receiver respectively. The propagation path of the generated wave is a function of the pump wave frequencies. It is assumed that the travel path change is small. Thus, the attenuation due to travel path change is neglected.

\subsection{Results and discussion}

\subsubsection{Analytical results}

The solution of Equation 4.15 is calculated as a function of the frequency of pump wave two $\left(f_{2}\right)$ while frequency $f_{1}$ is left constant. This is done for many different constant values of $f_{1}$ pump frequencies, ranging from $300 \mathrm{kHz}$ to $1.5 \mathrm{MHz}$, with the correspondent interaction angle $\alpha$ for each $f_{1}$. The calculated propagation angle $\psi$ as a function of frequency $f_{2}$ and $\alpha$ is shown in Figure 4.7 a to Figure 4.7f. Similarly, in Figure 4.7a to Figure 4.7f the solution to Equation 4.15 is shown in the colour map for the different frequencies $f_{1}$ as a function of interaction angle $\alpha$ and the excitation frequency $f_{2}$. The amplitude of the generated wave is in arbitrary units, being dark blue low amplitude (0) and yellow for high amplitude (6).

A wide range of propagation directions can be seen in Figure 4.7. For instance, $\psi$ linearly increases as $\alpha$ increases and vice versa. The propagation angle linearly increases with an increase in frequency $f_{2}$, however with lower rate. Furthermore, if the incident wave frequency $f_{1}$ is small, the range of propagation directions decreases (see Figure 4.7a) compared to a higher $f_{1}$ frequencies. The increase in propagation is depicted in Figure 4.7a to Figure 4.7f on the left side of each figure. If $f_{1}$ increases, the propagation directions for $f_{2}<1 \mathrm{MHz}$ frequencies become available. Thus, the range of available propagation directions increases (see Figure 4.7f). The information in Figure 4.7 can be used to estimate the adequate pump frequencies for an experimental set-up based on the possible interaction angles that can be feasible to achieve. Once an interaction angle is fixed, the propagation direction can be modified by one of the two pump wave frequencies. This allows to have one single experimental set-up for the pump waves and only change the position of the receiver. This in turns reduces the complexity of an experimental set-up. 
In Figure 4.7a to Figure 4.7f, two main regions can be seen. The first has its maximum amplitude at $\alpha=45^{\circ}$ and the second has its maximum amplitude at $\alpha=$ $135^{\circ}$. The region with the highest amplitude is the interaction angle $\alpha=45^{\circ}$. For both regions the amplitude of the generated wave increases with an increase in pump frequency $f_{2}$. Additionally, in the region around interaction angle $\alpha=100^{\circ}$, the amplitude for all the range of pump frequencies $f_{2}$ is the lowest. For the case of pump frequency $f_{1}=300 \mathrm{kHz}$ and at pump frequencies $f_{2}<1 \mathrm{MHz}$, the amplitude of the generated wave is the lowest for all interaction angles (see Figure 4.7a).

(a), $\mathrm{f}_{1}=300 \mathrm{kHz}$

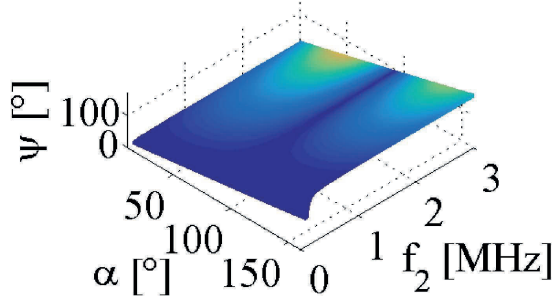

(c), $\mathrm{f}_{1}=800 \mathrm{kHz}$

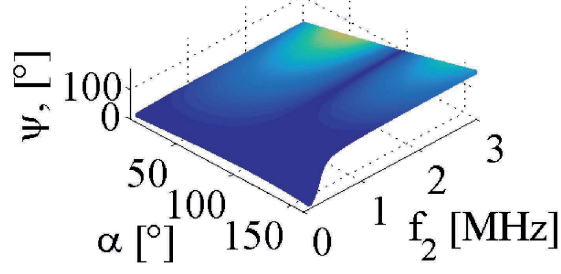

(e), $\mathrm{f}_{1}=1.3 \mathrm{MHz}$

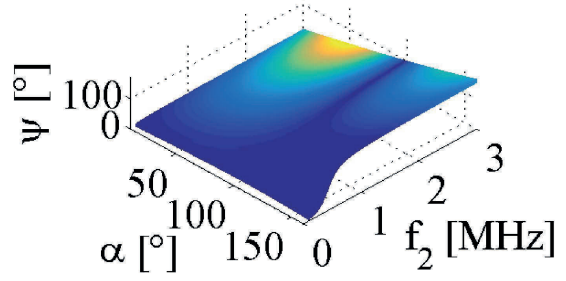

(b), $\mathrm{f}_{1}=550 \mathrm{kHz}$

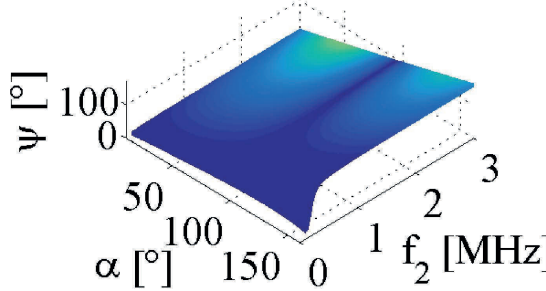

(d), $\mathrm{f}_{1}=1.05 \mathrm{MHz}$
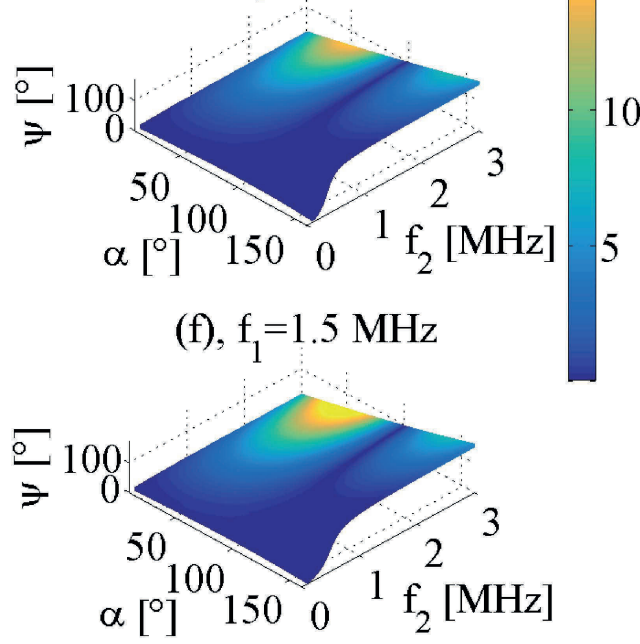

Figure 4.7

Propagation angle $\psi$ as a function of the interaction angle $\alpha$ and $f_{2}$ at $f_{1}$ equal to: (a) $300 \mathrm{kHz}$, (b) $550 \mathrm{kHz}$, (c) $800 \mathrm{kHz}$, (d) $1.05 \mathrm{MHz}$, (e) $1.3 \mathrm{MHz}$, (f) $1.5 \mathrm{MHz}$. The colour map represents the amplitude in arbitrary units (Equation 4.15).

As the pump frequency $f_{1}$ is increased, the amplitude decreases around the interaction angle $\alpha=135^{\circ}$. Thus, with higher pump frequency $f_{2}$ and higher pump frequencies $f_{1}$, the maximum amplitude of the generated wave is found at an 
interaction angles of $\alpha=45^{\circ}$ (see Figure 4.7f). For any pump frequency value $f_{1}$ the amplitude of the generated wave is extremely low at $f_{2}<1 \mathrm{MHz}$ (see Figure 4.7a to Figure 4.7f). In order to generate a high amplitude acoustic wave from the mixing of two incident waves, the frequency $f_{2}$ must be higher than $1 \mathrm{MHz}$. For the remainder of the experiments, $f_{1}$ is chosen to be $650 \mathrm{kHz}$, while $f_{2}$ is varied between $850 \mathrm{kHz}$ and $1.35 \mathrm{MHz}$. The calculated interaction depth is $2 \mathrm{~mm}$.

\subsubsection{Experimental results}

A typical recorded signal is shown in the time domain in Figure 4.8a. This signal is from the experiment with $f_{2}=1100 \mathrm{kHz}$. Figure $4.8 \mathrm{~b}$ depicts the corresponding frequency spectrum.

(a)

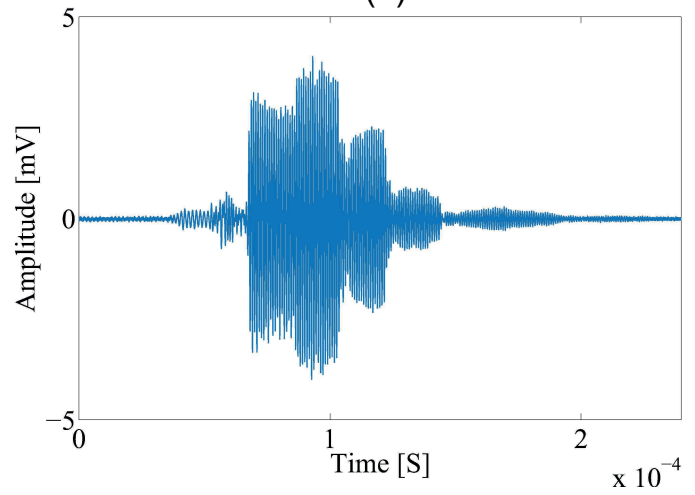

(b)

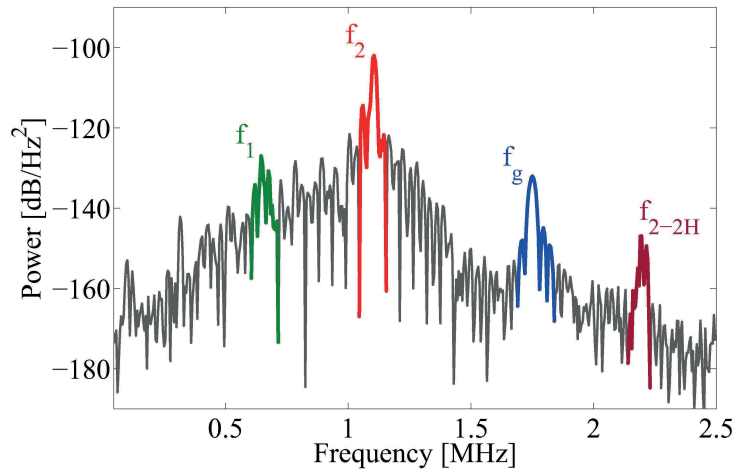

Figure 4.8

(a) Time domain raw data from the experiments with $f_{2}(=1100 \mathrm{kHz})$; (b) the frequency spectrum of the respective signal $f_{g}(=1.75 \mathrm{MHz})$.

In the frequency domain, four frequency components are shown. The frequency components expected are $f_{1}, f_{2}$ and $f_{g}$. Another frequency is found at $f_{2-2 H}$. The latter corresponds to the second harmonic of the second pump wave. No further 
harmonics are present in the frequency spectrum. The amplitude of the generated wave can be extracted in the time domain with a band-pass filter. However, in this research, the extraction is done in the frequency domain. The maximum amplitude of the generated wave was extracted from the component $f_{g}$ as depicted in Figure 4.8. There is a difference of approximately $30 \mathrm{~dB}$ between the amplitude of the second pump wave and that of the generated wave. A difference of approximately $5 \mathrm{~dB}$ was found between the first pump wave and the generated wave. The difference between the pump waves and the generated wave are due to the positioning of the receiver. A similar difference in amplitude is seen with the rest of the $f_{2}$ pump wave frequencies.

(a)

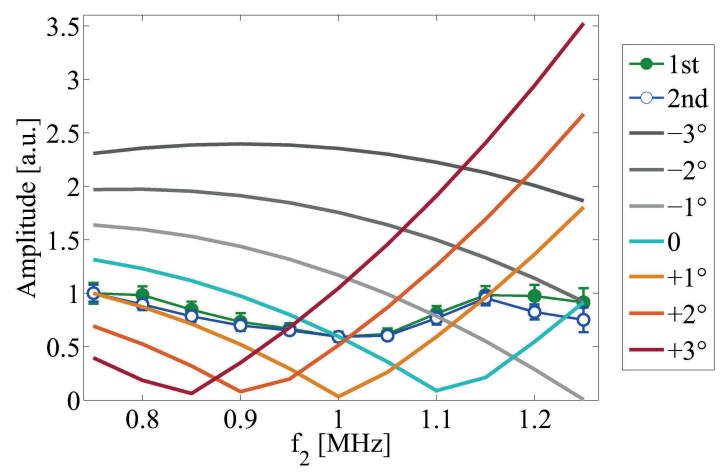

(b)

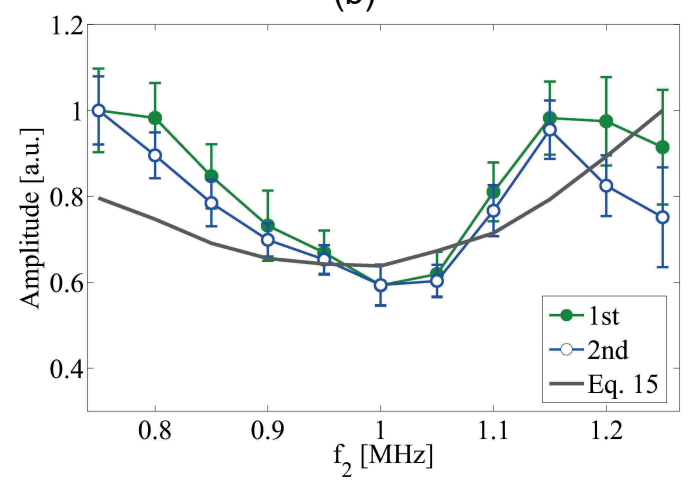

Figure 4.9

(a) Amplitude of the experimental results and the solution of Equation 4.15 for $\alpha$ $=98^{\circ}, \pm 1,2,3^{\circ}$. (b) Amplitude of the experimental results and the average of the solution of Equation 4.15.

The amplitudes of the measured signals as a function of $f_{2}$ are shown in Figure 4.9a and Figure 4.9b together with the solution of Equation 4.15. Solving Equation 4.15 for only one interaction angle $\alpha$ does not account for beam divergence nor for the beam width. In the experiments, however, there is beam divergence with a finite width, so many interactions happen simultaneously even at conditions 
where the theory predicts the contrary. Equation 4.15 was solved for $\pm 3^{\circ}$ with respect to the interaction angle $\alpha=98^{\circ}$ (see Figure 4.9a). The solution of the 7 interaction angles $\left(98^{\circ}, \pm 3^{\circ}\right)$ was averaged and is depicted in Figure $4.9 \mathrm{~b}$. The experiments correlate with the averaged analytical solution. A minimum value in both the experiments and analytic solution can be seen at a frequency $f_{2}=$ 1.1 MHz. The experimental results show that the amplitude of the generated wave was never zero. No signal could be recorded after a frequency above $f_{2}=$ $1.35 \mathrm{MHz}$, thus the analytical solution shown is also reduced to the range of 0.8 to $1.35 \mathrm{MHz}$.

\subsection{Conclusions}

In this research a solution of the scattering wave, generated from the mixing of two incident waves, is presented for the case where the angle of interaction is kept constant. The propagation direction of the generated wave is then steered by controlling the incident wave's excitation frequencies rather than the angle between the incident waves. This novel approach significantly reduces the complexity of a test set-up. Rather than adjusting the positions of three sensors, only the receiver has to be adjusted. Another advantage of this solution is the possibility to adjust the excitation frequencies to obtain the maximum amplitude of the generated wave. This reduces the effects of positioning errors. It is of great advantage for NDE applications were several conditions restrict the testing configuration.

In previous research, the range of excitation frequencies are limited to the space and accessibility to the material. In this research, this is not a limitation anymore and a broader range of pump frequencies are available. The model used to demonstrate the proposed approach has been verified with some experiments, which confirms the potential of the method.

\section{ACKNOWLEDGMENT}

This work was performed in the cooperation framework of Wetsus, European Centre of Excellence for Sustainable Water Technology (www.wetsus.eu). Wetsus is co-funded by the Dutch Ministry of Economic Affairs and Ministry of Infrastructure and Environment, the Province of Fryslân, and the Northern Netherlands Provinces. The authors like to thank the participants of the research theme "Smart Water Grids" for the fruitful discussions and their financial support. The authors thank to Hakan Kandemir for his help with the analytical solutions. 


\section{REFERENCES}

1. S. Mezil, N. Chigarev, V. Tournat, and V. Gusev, 'Evaluation of crack parameters by a nonlinear frequency-mixing laser ultrasonics method,' Ultrasonics, vol. 69, pp. 225-235, 2016.

2. H. Lv, J. Jiao, B. Wu, and C. He, 'Evaluation of Fatigue Crack Orientation Using Non-collinear Shear Wave Mixing Method,' J. Nondestruct. Eval., vol. 37, no. 4, pp. 1-16, 2018.

3. J. Jiao, J. Sun, N. Li, G. Song, B. Wu, and C. He, 'Micro-crack detection using a collinear wave mixing technique,' NDT E Int., vol. 62, pp. 122-129, 2014.

4. M. Sun, Y. Xiang, M. Deng, J. Xu, and F. Z. Xuan, 'Scanning non-collinear wave mixing for nonlinear ultrasonic detection and localization of plasticity,' NDT E Int., vol. 93, no. May 2017, pp. 1-6, 2018.

5. A. Demčenko, R. Akkerman, P. B. Nagy, and R. Loendersloot, 'Non-collinear wave mixing for non-linear ultrasonic detection of physical ageing in PVC,' NDT E Int., vol. 49, pp. 34-39, 2012.

6. C. Pecorari, 'Modeling non-collinear mixing by distributions of clapping microcracks,' Wave Motion, vol. 59, pp. 69-80, 2015.

7. G. Tang, M. Liu, L. J. Jacobs, and J. Qu, 'Detecting localized plastic strain by a scanning collinear wave mixing method,' J. Nondestruct. Eval., vol. 33, no. 2, pp. 196-204, 2014.

8. E. Escobar-Ruiz, A. Ruiz, W. Hassan, D. C. Wright, I. J. Collison, P. Cawley, and P. B. Nagy, 'Non-linear ultrasonic NDE of titanium diffusion bonds,' J. Nondestruct. Eval., vol. 33, no. 2, pp. 187-195, 2014.

9. Z. Zhang, 'Nonlinear Assessment of Material and Interface Imperfections Based on Non-Collinear Shear Wave Mixing,' 2016.

10. Z. Zhang, P. B. Nagy, and W. Hassan, 'Analytical and numerical modeling of non-collinear shear wave mixing at an imperfect interface,' Ultrasonics, vol. 65, pp. 165-176, 2016.

11. T. Ju, J. D. Achenbach, L. J. Jacobs, and J. Qu, 'A non-collinear mixing technique to measure the acoustic nonlinearity parameter of an adhesive bond from one side of the sample,' AIP Conf. Proc., vol. 1806, 2017.

12. A. Demčenko, L. Mainini, and V. A. Korneev, 'A study of the noncollinear ultrasonic-wave-mixing technique under imperfect resonance conditions,' Ultrasonics, vol. 57, pp. 179-189, 2015.

13. M. McGovern and H. Reis, 'Damage characterization in dimension limestone cladding using noncollinear ultrasonic wave mixing,' Opt. Eng., vol. 55, no. 1, p. 011012, 2015.

14. S. K. Chakrapani and D. J. Barnard, 'Determination of acoustic nonlinearity parameter $(\beta)$ using nonlinear resonance ultrasound spectroscopy: Theory and experiment,' J. Acoust. Soc. Am., vol. 141, no. 2, pp. 919-928, 2017.

15. M. Liu, G. Tang, L. J. Jacobs, and J. Qu, 'Measuring acoustic nonlinearity parameter using collinear wave mixing,' J. Appl. Phys., vol. 112, no. 2, 2012.

16. L. H. Taylor and F. R. Rollins, 'Ultrasonic study of three-phonon interactions. I. Theory,' Phys. Rev., vol. 136, no. 3A, 1964.

17. A. C. Holt and J. Ford, 'Theory of ultrasonic three-phonon interactions in single-crystal solids,' J. Appl. Phys., vol. 40, no. 1, pp. 142-148, 1969. 
18. G. L. Jones and D. R. Kobett, 'Interaction of Elastic Waves in an Isotropic Solid,' J. Acoust. Soc. Am., vol. 35, no. 1, pp. 5-10, 1963.

19. Y. Hiki and K. Mukai, 'Ultrasonic Three-Phonon Process in Copper Crystal,' J. Phys. Soc. Japan, vol. 34, no. 2, pp. 454-461, 1973.

20. V. A. Korneev and A. Demčenko, 'Possible second-order nonlinear interactions of plane waves in an elastic solid,' J. Acoust. Soc. Am., vol. 135, no. 2, pp. 591-598, 2014. 


\title{
Chapter 5

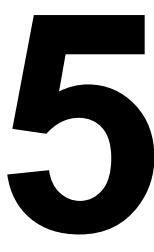

\section{Power spectral analysis of a one-sided non-collinear wave mixing test in a PVC pipe}

\begin{abstract}
The signal strength and signal-to-noise (SNR) ratio of the generated wave from a one-sided versus dual-sided wave mixing configuration were investigated. The experimental scheme is divided in two parts. The first set of experiments were performed in manufactured PVC samples. The second set of experiments were performed in a field PVC pipe taken out from the drinking water network in The Netherlands. The generated wave power spectrum amplitude from the two-sided configuration is $4 \mathrm{~dB}$ higher than the one-sided configuration. Furthermore, the SNR from the field tests are as high as the ones from the laboratory tests. Thus, the one-sided wave mixing configuration can be used for the inspection of PVC pipes in service. The major consideration is the proper sensor alignment with respect to the longitudinal direction of the pipe, this requires more development.
\end{abstract}

This chapter is in submission process as: Delgadillo, H. H., Chidhambaram, N., Loendersloot, R., Yntema, D., Tinga, T., \& Akkerman, R. (2019). Power spectral analysis of a one-sided non-collinear wave mixing test in a PVC pipe. 


\subsection{Introduction}

The use of polyvinyl chloride (PVC) in the drinking water sector began in the 1940s in many European countries. The lifetime of plastic pipes is estimated to be above 50 years [1-3]. Nonetheless, some study cases have shown pipe failure not long after installation $[4,5]$. In the last decades, the interest in developing nondestructive inspection methodologies from the water utilities increased. The main requirement from the water utilities is that the inspection methodologies should be capable of detecting changes in the condition of PVC pipes.

Available inspection technologies of PVC pipes are mostly focused on sewage pipes [4-8]. Amongst the most used technologies, the camera inspection is the method of choice for many companies. This allows to check for many defects such as dents, cracks, ovality and pipe deflection $[4,6,7]$. This technology provides information on the pipe surface as well as the presence of sedimentation. More advanced technologies often combine the CCTV set-up with a laser profiling system [6, 9]. The addition of this system is the measurement of surface roughness and the generation of 3-D images of the pipe geometry. However, it is still only limited to surface information. These two technologies require access to the interior of the network without digging the pipes out of the ground, although access to the grid from at least one point is necessary to launch the respective tools. An inline inspection system can carry out versatile measurements, however, up till today there is no measurement system capable of detecting ageing in PVC drinking water pipes [8].

Demčenko et al. [10] found that non-linear ultrasonics are sensitive to ageing in PVC pipes. In their study, they demonstrated that the amplitude of a wave generated from the interaction of two incident waves is sensitive to ageing in PVC. Furthermore, Visser et al. [3] developed an in-site non-destructive technique to measure ageing based on micro-indentation test. Their results showed that there is a correlation between hardness measurements and ageing in PVC. Still, there was high uncertainty in the results. Natural ageing of PVC is the main mechanism that contributes to the deterioration of mechanical performance of buried PVC infrastructure [3, 11-13].

Form these latter two measurement techniques (wave mixing and microindentation), nonlinear ultrasonics seems more feasible to implement in an inline inspection tool. Implementing such a technique for the inspection of PVC drinking water pipes seems very promising. The dual sided access to the surfaces and the specimen's geometry are a few factors that restrict its applicability.

The first objective of this research is to demonstrate that minimal changes in the composition of PVC have an effect on the propagation of acoustic waves in PVC materials. The second objective is to compare the signal strength and the signalto-noise ratio (SNR) of a one-sided and a two-sided wave mixing configuration. This is performed in the laboratory on flat manufactured specimens. The third objective is to compare the SNR of measurements on field tests pipes with the 
ones from the laboratory. This was performed in a pipe taken from service by drinking water supplier Evides in The Netherlands.

\subsubsection{Polyvinyl chloride pipes}

Polyvinyl chloride was firstly manufactured and installed for the distribution of drinking water in the 1940s in many European countries [1-3]. The advantage of plastic pipes over ferrous and cementitious ones is mostly the resistance to the aggressive environment. The aggressive environment in the supply of drinking water comes from a low $\mathrm{pH}$ soil, rain water and the low ion content of the conveyed drinking water $[14,15]$. The effect of the latter on ferrous and cementitious pipes is material loss and thus a reduction in strength. The lifetime of plastic pipes is estimated to be 50 years, thus, more attractive and with much lower cost than the conventional pipes at that time. Nonetheless, PVC pipes have occasionally failed on a very early stage [5]. The major reason for this is because of its brittle nature. Thus, they are quite susceptible to impact during the installation and during operation. In the 1970's a new type of PVC was developed which had a different composition and additives that made it more ductile and resistant to impacts [3, 13]. The characteristics that have a major influence on the mechanical behaviour of PVC materials are the level of gelation, the additive content and the molecular weight $[1,3,13]$.

The efforts to increase the mechanical performance of polyvinyl chloride pipes did not cease with the latter development. A good example of this is the introduction of uniaxial and biaxial oriented PVC pipes. In the United Kingdom and in Australia a new manufacturing process was introduced which allowed to arrange the molecular orientation in a uni-directional and bi-directional configuration $[16,17]$. By aligning the molecular chains of the polymer and stacking-like laminates of oriented sheets, it is possible to enhance the mechanical performance of PVC pipes. The main advantage of such configurations is the possibility to control the propagation direction of cracks $[16,17]$.

In a drinking water network that has been in service for half a century, many types of PVC pipes can be found. The great variety of materials within the network makes the development of an inspection technique even more challenging, especially because there is no reference (initial condition parameters) standards of each material installed. In this research, three different types of PVC are used in order to characterize the response of acoustic waves.

\subsubsection{The non-collinear wave mixing principle}

In a solid material, the local interaction of two incident acoustic waves from two different sources may generates a third acoustic wave as depicted in Figure 5.1 [10], [18-21]. These acoustic waves can propagate either in compressional or shear mode in direction $\mathbf{k}_{1}$ and $\mathbf{k}_{2}$ (as shown in Figure 5.1). The generated 
acoustic wave $\mathbf{k}_{g}$ propagates at an angle $\psi$ with respect to the direction of the incident acoustic wave $\mathbf{k}_{1}$.

Two conditions have to satisfied for the generation of a third acoustic wave. The first is, that the wave can either be the sum or the difference in frequency

$$
\omega_{g}=\omega_{1} \pm \omega_{2}
$$

where $\omega_{1}$ and $\omega_{2}$ are the pump wave frequencies respectively and $\omega_{g}$ is the frequency of the generated wave. The second condition is that

$$
\mathbf{k}_{g}=\mathbf{k}_{1} \pm \mathbf{k}_{2}
$$

where $\mathbf{k}_{1}$ and $\mathbf{k}_{2}$ are the incident wave vectors respectively and $\mathbf{k}_{g}$ is the vector of the generated wave and $k_{n}=\omega_{n} / v_{n}$; where $v_{n}$ is the speed of sound in the solid material.

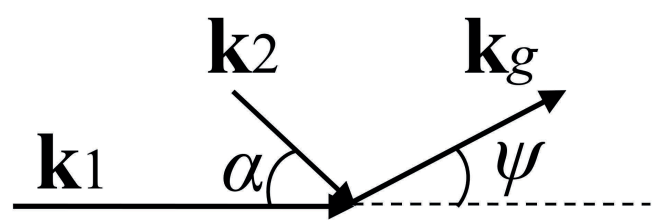

Figure 5.1

Generated wave from the interaction of two incident waves.

The amplitude of the generated wave is dependent on the angle between the incident waves, better known as the interaction angle $\alpha$. The interaction angle $\alpha$ is defined by the cosine law

$$
\left(\frac{\omega_{1}}{v_{1}}\right)^{2}+\left(\frac{\omega_{2}}{v_{2}}\right)^{2} \pm 2 \frac{\omega_{1} \omega_{2}}{v_{1} v_{2}} \cos \alpha=\left(\frac{\omega_{1} \pm \omega_{2}}{v_{g}}\right)^{2}
$$

where $v_{1}$ and $v_{2}$ are the speed of sound in the medium, either longitudinal or shear. The propagation angle of the generated wave is obtained with respect to the direction of $\mathbf{k}_{1}$ as

$$
\psi=\tan ^{-1}\left(\frac{ \pm \frac{v_{1}}{v_{2}} d \sin \alpha}{1 \pm \frac{v_{1}}{v_{2}} d \cos \alpha}\right)
$$

where $d=\omega_{2} / \omega_{1}$. The speed of sound in the material for longitudinal and transverse waves can be estimated from the material properties $[22,23]$

$$
v_{l}=\left(\frac{\lambda+2 \mu}{\rho}\right)^{1 / 2}=\left(\frac{(1-v)}{(1+v)(1-2 v)} \frac{E}{\rho}\right)^{1 / 2}
$$




$$
v_{t}=\left(\frac{\mu}{\rho}\right)^{1 / 2}=\left(\frac{1}{2(1+v)} \frac{E}{\rho}\right)^{1 / 2}
$$

where $\lambda$ is the first Lamé constant and $\mu$ is the second Lamé constant or shear modulus; $\rho$ is the material density; $v$ is the Poisson's ratio and $E$ is the Young's modulus. These parameters can be found in literature. However, in practice they might differ. If for instance the Young's modulus is available in literature, the speed of sound can be estimated as well as shown in Equation 5.5 and Equation 5.6. If, however, only the Lamé constants are available, the Poisson's ratio and the Young's modulus are estimated as [22, 23]

$$
\begin{gathered}
v=\frac{\lambda}{2(\mu+\lambda)} \\
E=\frac{\mu(3 \lambda+2 \mu)}{\lambda+\mu}
\end{gathered}
$$

\subsection{Methodology}

This section is divided in the following parts. Firstly, the main characteristics of the PVC samples are described, followed by the characterization of the speed of sound in shear and longitudinal mode. The estimated speed of sound from literature is compared with the measured speed of sound. The non-collinear wave mixing test is then performed in the laboratory samples and in the field PVC pipe.

\subsubsection{PVC samples}

Two different PVC types were used with different molecular weight. Two different additive packages were used for the same molecular weight. Table 5.1 shows the specifications of the three different samples.

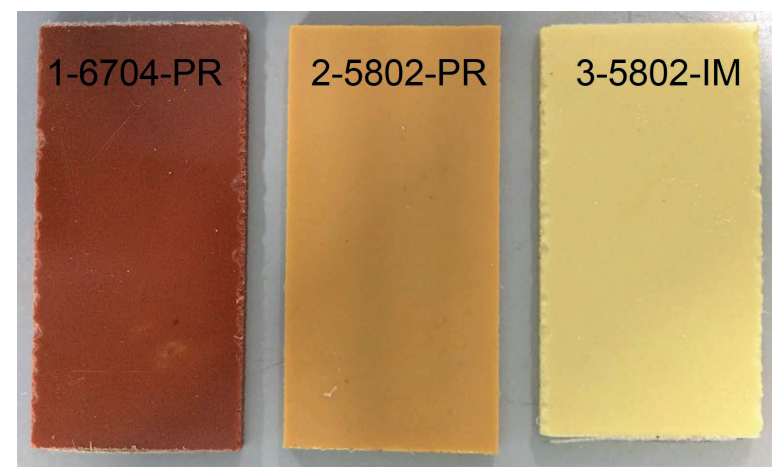

Figure 5.2

PVC specimens manufactured with different composition and colors 
Table 5.1 Material composition, mixture and end temperature

\begin{tabular}{|c|c|c|c|c|}
\hline Material & & Plate 1 & Plate 2 & Plate 3 \\
\hline PVC K67 & [phr] & 100 & & \\
\hline PVC K58 & [phr] & & 100 & 100 \\
\hline CalZn based additive & [phr] & 2,6 & 2,6 & \\
\hline Org. based additive & [phr] & & & 5,6 \\
\hline Chalk & [phr] & 4 & 4 & 1 \\
\hline Application & & Pressure pipe & Non-pressure fitting & Mixed \\
\hline
\end{tabular}

The polymer dry-blends were mixed in a planetary mixer. The samples were taken from the delivered packages without pre-treatment. The specimens have the following dimensions: 50x95x4 mm. These are shown in Figure 5.2.

\subsubsection{Longitudinal wave mode speed of sound}

A standard pulse-echo configuration was used to measure the speed of sound and reflected amplitudes in the longitudinal mode. The speed of sound is measured from the time difference in time-of-travel between the front wall and back wall reflections. The measured parameters in the longitudinal wave mode are shown in Figure 5.3.

(a)

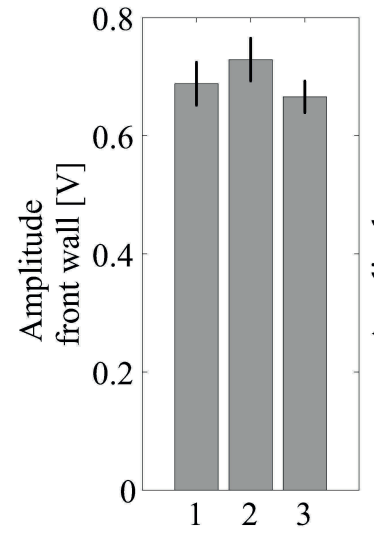

(b)

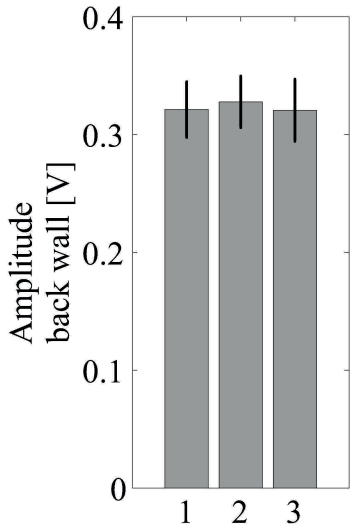

(c)



Figure 5.3

Longitudinal wave mode: (a) Amplitude of the specimen's front surface reflection; (b) amplitude of the specimen's back surface reflection; (c) measured speed of sound. (1) 1-6704-PR specimens; (2) 2-5802-PR specimens and (3) 3-5802-IM specimens. 
There is no significant change in the reflected amplitude from both the back surface (see Figure 5.3a) and front surface reflections (see Figure 5.3b) of the specimen. The longitudinal wave speed of sound of specimen 2-5802-PR is approximately $2 \%$ lower compared to the other two types of PVC. Since speed of sound changes with the properties of the material (and not with the amplitudes of the front and back wall reflections), it is known from this result that each of the PVC samples has slightly varying properties. This is attributed to the difference in the material compositions.

\subsubsection{Shear wave mode speed of sound}

The test set-up for the shear wave mode is depicted in Figure 5.4. The first critical angles for each type of material are $38.5^{\circ}, 39^{\circ}$ and $38^{\circ}$, based on the measured longitudinal speed of sound and Snell's law. A transducer (transmitter) is placed at $50^{\circ}\left(\theta_{i}\right.$ in Figure 5.4), well above the critical angle, to refract a shear wave mode only. A second transducer (receiver) with the same central frequency is placed with the same angle of inclination and at the same height $y$ from the specimen's surface.

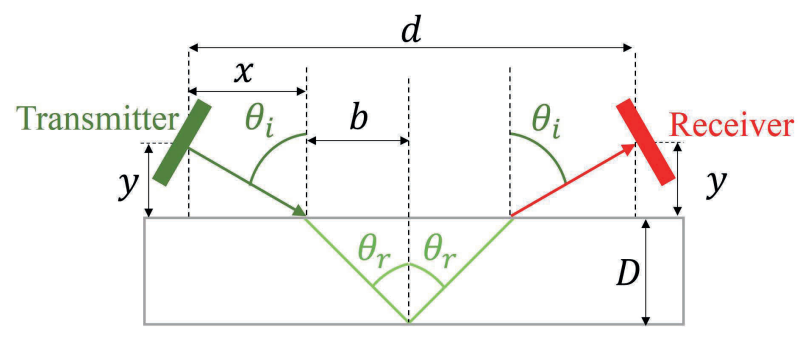

Figure 5.4

Experimental arrangement for measuring the shear speed of sound and transmitted/reflected amplitude

The receiver is moved horizontally until the maximum amplitude of the reflected shear wave from the back of the specimen is reached. The distance $d$ is measured and the shear speed of sound is calculated from

$$
\begin{aligned}
& d=2 b+2 x \\
& x=y \tan \theta_{i}
\end{aligned}
$$

From substituting Equation 5.9 in Equation 5.10 and the refracted angle (Figure 5.4):

$$
\begin{aligned}
& b=\frac{d-2 y \tan \theta_{i}}{2} \\
& \theta_{r}=\tan ^{-1}\left(\frac{b}{D}\right)
\end{aligned}
$$


The shear velocity in the material $v_{s m}$, is then calculated from Snell's law:

$$
v_{s m}=v_{w} \frac{\sin \theta_{r}}{\sin \theta_{i}}
$$

where $v_{w}$ is is the propagation velocity in water. From substituting Equation 5.11 in Equation 5.12 and in then in Equation 5.13, the following equation is obtained:

$$
v_{s m}=v_{w} \frac{\sin \left(\tan ^{-1}\left(\frac{d-2 y \tan \theta_{i}}{2 D}\right)\right)}{\sin \theta_{i}}
$$

The measured parameters in the shear wave mode are shown in Figure 5.5. The amplitude of the 1-6704-PR specimen's front surface reflection is approximately $50 \%$ lower than the amplitude of the front surface reflection of the other two PVC types (Figure 5.5b).
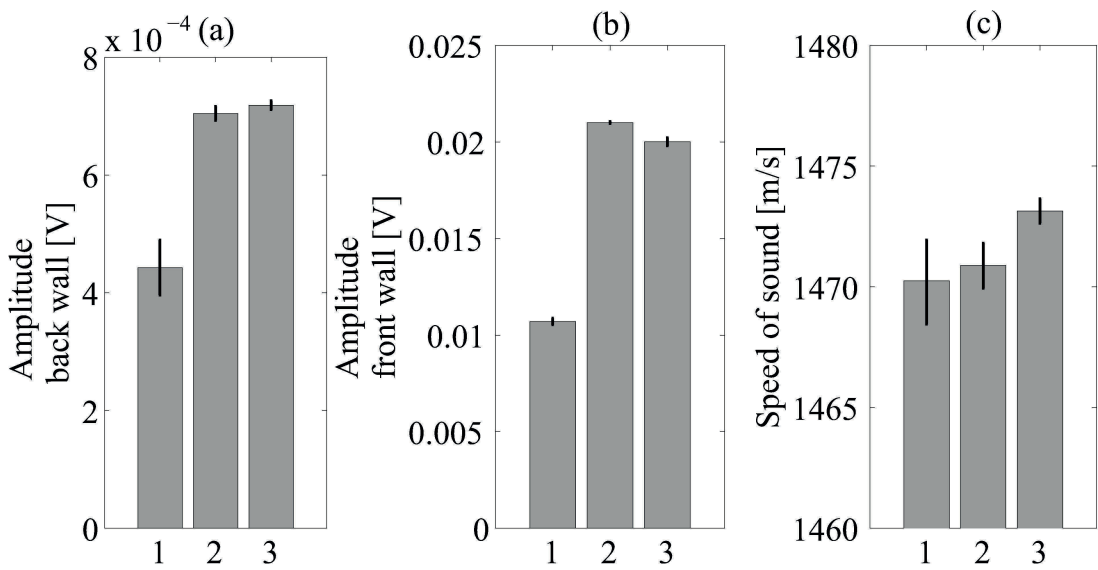

Figure 5.5

Shear wave mode: (a) Amplitude of the specimen's back surface reflection; (b) amplitude of the specimen's front surface reflection; (c) measured speed of sound.

(1) Is 1-6704-PR specimens; (2) is 2-5802-PR specimens and (3) is 3-5802-IM specimens.

The amplitude of the 1-6704-PR specimen's back surface reflection is approximately $35 \%$ lower than that of the other two PVC types (Figure 5.5a). The shear wave velocity of sound of the three PVC types does not significantly change (as calculated from Equation 5.14). The additives and composition of the PVC samples have an effect on the transmission and reflection energy between water-PVC interfaces as well as in the speed of sound.

Equations 5.5 to 5.8 give correct values of the PVC whereas Equations 5.9 to 5.14 give unrealistic material properties. The estimated linear properties are compared with the literature in Table 5.2. The parameters estimated for the column 
"literature" are directly from Equations 5.5-5.8. The parameters from columns 16704-PR, 2-5802-PR and 3-5802-IM are first calculated from Equations 5.9-5.14; thereafter Equations 5.5 and 5.6 are solved for $E$ and $v$ and lastly Equations 5.7 and 5.8 are solved for $\mu$ and $\lambda$.

Table 5.2 Linear parameters from PVC estimated from measured speed of sound and from literature [19], [24]

\begin{tabular}{|c|c|c|c|c|}
\hline & Literature & 1-6704-PR & 2-5802-PR & 3-5802-IM \\
\hline$v$ & 0.332 & 0.195 & 0.177 & 0.201 \\
\hline$E(\mathrm{GPa})$ & 4.87 & 6.97 & 6.88 & 7.04 \\
\hline$\lambda(\mathrm{GPa})$ & 3.64 & 1.87 & 1.61 & 1.98 \\
\hline$\mu(\mathrm{GPa})$ & 1.83 & 2.91 & 2.92 & 2.92 \\
\hline$\rho(\mathrm{kg} / \mathrm{m} 3)$ & 1350 & 1350 & 1350 & 1350 \\
\hline$v_{l}(\mathrm{~m} / \mathrm{s})$ & 2,325 & 2,390 & 2,350 & 2,410 \\
\hline$v_{s}(\mathrm{~m} / \mathrm{s})$ & 1,164 & 1,470 & 1,471 & 1,473 \\
\hline
\end{tabular}

The calculated speed of sound in the longitudinal mode has a reasonable deviation from the literature, which is approximately $2.8 \%, 1 \%$ and $3.5 \%$ for the samples with material composition 1-6704-PR, 2-5802-PR and 3-5802-IM respectively. The contrary happens with the measured speed of sound in the shear wave mode where the deviations are $26.2 \%, 26.3 \%$ and $26.5 \%$ for the samples with material composition 1-6704-PR, 2-5802-PR and 3-5802-IM respectively. The major cause for such a deviation is due to the distance $y$ from the surface of the sensor (Equation 5.14) and the measured distance $d$ (Equation 5.14) from the centre of the face of each sensor (see Figure 5.4). The sensitivity of these parameters is shown in Figure 5.6.

The errors induced in the measurement distance $d$ and $y$ are quite large as a $3 \%$ in $d$ value induces a $10 \%$ in the speed of sound and similarly a $3 \%$ change in the measured distance $y$ induces a $10 \%$ in the speed of sound. Hence, it can be concluded that accurately estimating the shear velocity is more challenging than estimating the longitudinal velocity. This has immediate consequence for using the shear wave mode as field measurements inherently show positional variations of the transducers with respect to the material to be measured. The actual longitudinal wave velocity may be retrieved from such a measurement with reasonable accuracy, it is unlikely that an accurate estimation of the shear wave velocity can be obtained. The effect of estimating inaccurate shear wave velocity on the wave mixing configuration will be further discussed in the following section. 




Figure 5.6

Sensitivity of the measured parameter $d$, the distance $y$ from the first surface of the sensor to the central point of the transducer surface and the incident angle $\theta_{i}$

\subsubsection{Non-collinear wave mixing}

The propagation direction $\psi$ is dependent on the solid material's linear elastic parameters. These parameters can be found in literature $[19,24]$ or can be experimentally characterized. In previous section, the longitudinal and shear speed of sound were measured. Prior to determine the adequate wave mixing configuration, the effect of measuring an inaccurate speed of sound on the propagation of the generated wave will be studied. Therefore, the propagation direction $\psi$, interaction angle $\alpha$ and orientation of receiver $\theta_{3}$ are compared with the parameters from Table 5.2. This comparison is shown in Table 5.3.

The orientation of sensor three with respect to a vertical line is

$$
\theta_{3}=\sin ^{-1}\left(\frac{v_{w}}{v_{l}} \sin \left(\theta_{r s 1}-\psi\right)\right)
$$

where $\theta_{r s 1}$ is the refracted shear wave component of the incident wave one. In Figure 5.7, the orientation of each sensor with respect to a vertical line is depicted.

Table 5.3 Interaction angle $\alpha$ (Equation 5.3), propagation direction $\psi$ (Equation 5.4) and the position of receiver $\theta_{3}$ (Equation 5.15)

\begin{tabular}{|c|c|c|c|c|}
\hline & Literature & 1-6704-PR & 2-5802-PR & 3-5802-IM \\
\hline$\alpha\left({ }^{\circ}\right)$ & 72.5 & 96.7 & 96.8 & 96.9 \\
\hline$\psi\left({ }^{\circ}\right)$ & 40 & 53.4 & 53.4 & 53.5 \\
\hline$\theta_{3}\left({ }^{\circ}\right)$ & 2.1 & 3.1 & 3.2 & 3.1 \\
\hline
\end{tabular}


The position of sensor three $\theta_{3}$ differs by approximately $1^{\circ}$ with respect to the three different types of specimens. In section 5.2.3 it was shown that the measurement set-up for shear speed of sound is very sensitive to errors and leads to unrealistic linear properties $(E, \mu, \lambda, v)$. However, the error of measuring inaccurate shear speed of sound has a small effect on the position of the receiver.

Based on the material properties from literature, the positioning of the two transmitters and the two receivers were calculated. The two pump waves are transmitted in the longitudinal mode in water. Refraction of the longitudinal waves occurs when they reach the first surface. Only shear wave components are refracted and propagated in the PVC material. This process is depicted in Figure 5.7. Two transmitters are used for the generation of the scattering wave for the shear + shear interaction process $\left(S_{1}+S_{2}=L\right)$ and two receivers are placed at locations where the longitudinal scattering wave is expected to arrive.

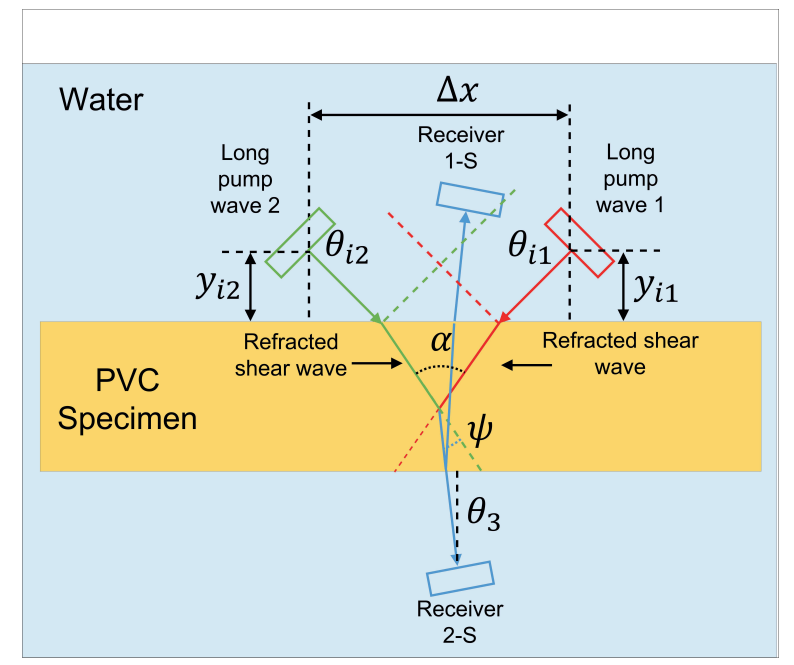

\section{Figure 5.7}

Sensor positioning for shear wave + shear wave interaction process. Test set-up was submerged in a water container during the experiments.

Receiver $1-S$ is the transducer placed on the same side where the sending transducers are placed. Receiver 2-S is placed on the other side of the specimen as shown in Figure 5.7. One-sided non-collinear wave mixing has been studied for Titanium and aluminium plates [21, 25]. The positioning of the sensors is shown in Table 5.4.

Table 5.4 Set-up configuration and sensor positioning

\begin{tabular}{ccccccc}
\hline $\begin{array}{c}y_{i 2} \\
{[\mathrm{~mm}]}\end{array}$ & $\begin{array}{c}y_{i 1} \\
{[\mathrm{~mm}]}\end{array}$ & $\begin{array}{c}\theta_{i 2} \\
{\left[{ }^{\circ}\right]}\end{array}$ & $\begin{array}{c}\theta_{i 1} \\
{\left[{ }^{\circ}\right]}\end{array}$ & $\begin{array}{c}\theta_{3} \\
{\left[{ }^{\circ}\right]}\end{array}$ & $\begin{array}{c}\Delta x \\
{[\mathrm{~mm}]}\end{array}$ & $\begin{array}{c}\alpha \\
{\left[{ }^{\circ}\right]}\end{array}$ \\
\hline 24.3 & 21 & 50.7 & 47.5 & 2.5 & 57.1 & 72.5 \\
\hline
\end{tabular}


For each type of specimen an optimization of the amplitude strength was performed by changing the pump wave frequencies $[18,19]$. With this procedure, the differences due to material property variations are compensated. The selected set of frequencies for each type of specimen is shown in Table 5.5.

Table 5.5 Pump waves frequency settings for the three types of PVC

\begin{tabular}{|l|c|c|c|}
\hline \multicolumn{1}{|c}{} & \multicolumn{1}{c}{ 1-6704-PR } & 2-5802-PR & 3-58-02-IM \\
\cline { 2 - 4 }$f_{1}, \mathrm{MHz}$ & 1.240 & 1.250 & 1.210 \\
\cline { 2 - 4 }$f_{2}, \mathrm{MHz}$ & 1.060 & 1.030 & 0.900 \\
\cline { 2 - 4 }$f_{g}, \mathrm{MHz}$ & 2.300 & 2.280 & 2.110 \\
\hline
\end{tabular}

Fine adjustments of the incident angles were performed until the maximum amplitude of the generated wave is obtained in each type of specimen. Similarly, the alignment of the receiver was adjusted until the maximum amplitude was obtained. With this procedure the interaction of interest is captured with maximum amplitude.

The sensors used were two broadband $1 \mathrm{MHz}$ central frequency transducers (transmitters), and two broadband $2.25 \mathrm{MHz}$ central frequency transducers (receivers). An input voltage with 30 sinusoidal cycles (lowest frequency) and 40 cycles (highest frequency) were used as the input waves. Below these number of cycles, the energy was too low and a generated wave could not be clearly found. A larger number of cycles is used for the highest frequency in order to increase interaction with maximum available area. The amplitude of the generated wave was recorded with a 15-bit resolution and $125 \mathrm{MS} / \mathrm{s}$ acquisition rate. The maximum amplitude of each frequency component $\left(f_{1}, f_{2}\right.$ and $\left.f_{g}\right)$ in the frequency domain was extracted. The average and standard deviation were calculated. The configuration of the electronics and sensors is depicted in Figure 5.8a and the positioning of the sensors is depicted in Figure $5.8 \mathrm{~b}$. The equipment brand and type is shown in Table 5.6.
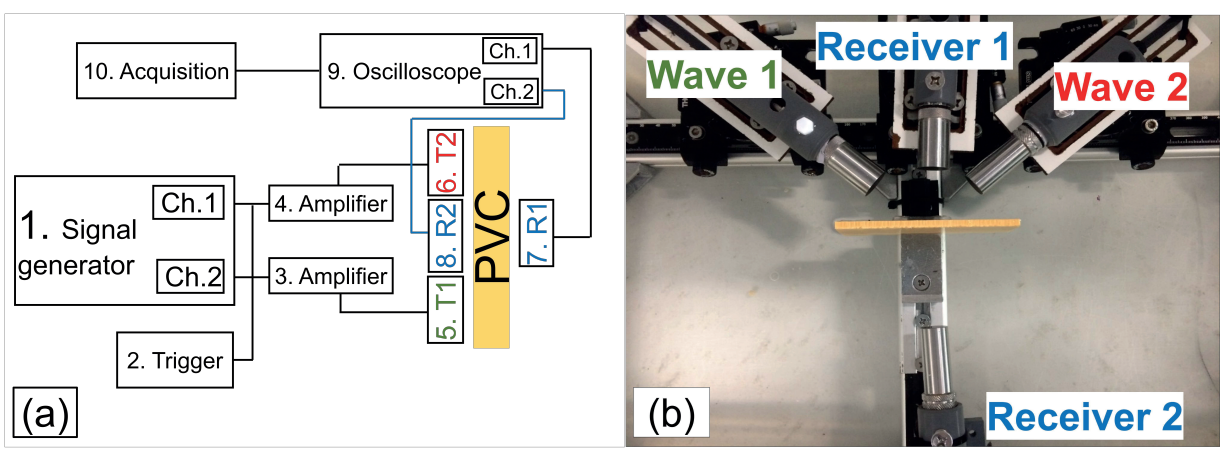

Figure 5.8

Depicts (a) electronic equipment and (b) sensor positioning test set-up. 
Table 5.6 Electronic components and sensors used

\begin{tabular}{|c|c|c|c|c|}
\hline $\begin{array}{c}\text { 1. Signal } \\
\text { generator }\end{array}$ & 2. Trigger & 3. Amplifier & 4. Amplifier & 5. T 1 \\
\hline $\begin{array}{c}\text { Keysight } \\
\text { 33512B }\end{array}$ & $\begin{array}{c}\text { Aim-TTi TG } \\
2000\end{array}$ & $\begin{array}{c}\text { Tomco } \\
\text { BTM00250 }\end{array}$ & $\begin{array}{c}\text { Tomco } \\
\text { BTM00250 }\end{array}$ & $\begin{array}{c}\text { Sofranel } \\
\text { IBHG014 }\end{array}$ \\
\hline 6. T 2 & 7. R 1 & 8. R 2 & 9. Oscilloscope & 10. Acquisition \\
\hline $\begin{array}{c}\text { Olympus I4- } \\
\text { Olympus I4- }\end{array}$ & $\begin{array}{c}\text { Sofranel } \\
\text { IBHG024 }\end{array}$ & $\begin{array}{c}\text { Picoscope } \\
\text { 5442B }\end{array}$ & Computer \\
\hline
\end{tabular}

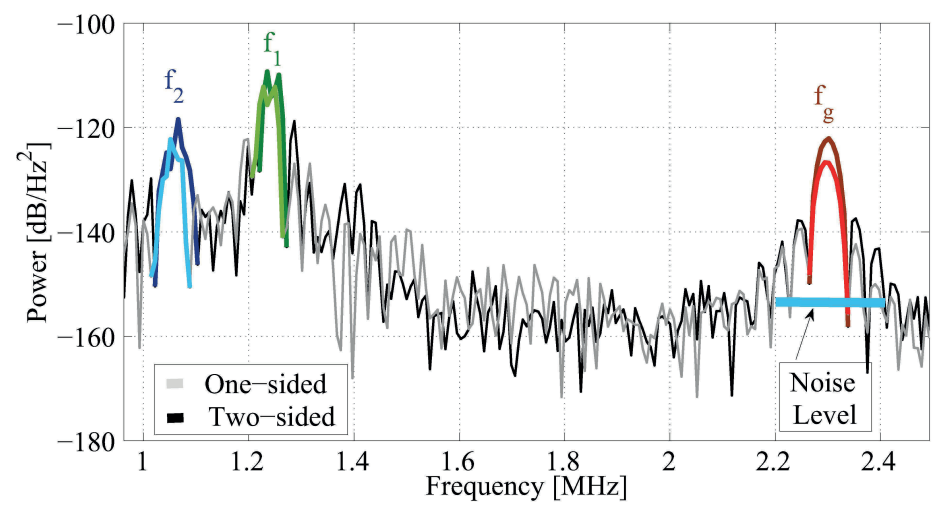

Figure 5.9

Frequency spectrum of the one-sided (light grey) and two-sided (black) wave mixing configurations. The frequency components in the darker colors are from the two-sided configuration and those from in lighter colors are from the one-sided configuration.

The signal-to-noise ratio is calculated from subtracting the power spectrum of the noise level to the power spectrum of the generated wave in the frequency domain at the particular frequency of interest. This calculation is performed for each type of PVC sample as well as for the field pipe. Furthermore, only for the PVC samples, the difference in power spectrum of the generated wave between the one-sided and the two-sided experiments is calculated as well. The amplitudes were extracted as follows. The data was transformed to the frequency domain with the Fast Fourier transform and the level at the frequencies $f_{1}, f_{2}$ and $f_{g}$ were extracted as depicted in Figure 5.9.

\subsubsection{Field test of PVC drinking water pipe}

Based on the calculations performed in section 5.2.4 for the interaction angle $\alpha$ and the propagation direction of the generated wave $\psi$, the configuration of the 
transmitters and receiver (one-sided) were defined. The configuration used for the field pipe test is shown in Figure 5.10. An optimization of the amplitude strength was performed by changing the pump wave frequencies as described in Section 2.4. The selected set of frequencies for the pipe are: $f_{1}=720 \mathrm{kHz}, f_{2}=940 \mathrm{kHz}$, $f_{g}=1.66 \mathrm{MHz}$.

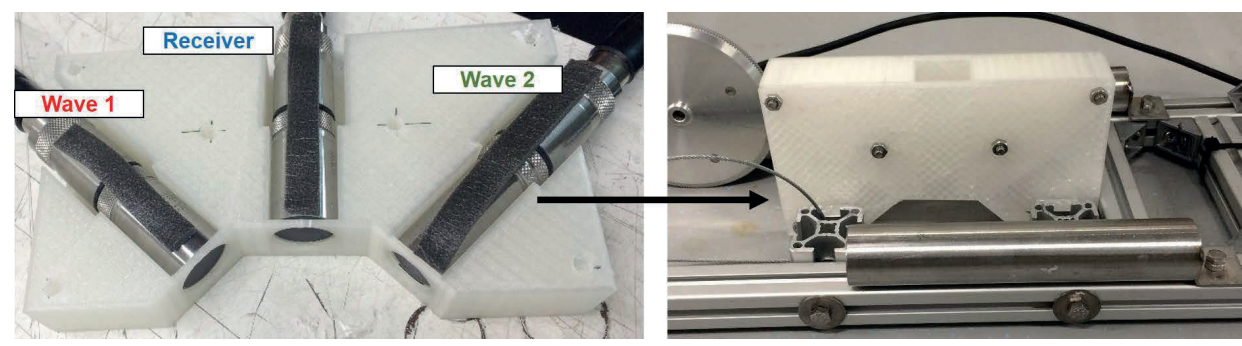

Figure 5.10

Holding fixture used in the mobile carrier inside the pipe. The space within the white fixture has the dimensions according to the angles of incidence and distances.

A 5-meter-long pipe with a $600 \mathrm{~mm}$ diameter and $16 \mathrm{~mm}$ thickness was tested with the non-collinear wave mixing technique. Four sections of this pipe were tested. Each section was tested two times. For this to work, a set-up that could move inside the pipe was built. The set-up is composed of an aluminium carrier that transports the ultrasonic sensors together with a wheel encoder as shown in Figure 5.11. The wheel encoder recorded the position as the carrier moved forward inside the pipe. The carrier is manually moved inside the pipe with the help of a cable connected to the front end of the fixture. At the rear of the fixture, another cable is used to pull back the carrier to its original position.

The inspected pipe can be seen in Figure 5.11a as well as the starting and ending point locations. In Figure 5.11b, the measurement setup is shown. The connexion of the electronic equipment and sensors is the comparable as in Figure 5.8a (without the Receiver 2). The equipment is described as follows: (1) triggers for the two amplifiers; (2) the signal generator for the excitation signals; (3) amplifiers; (4) synchronisation signal; (5) power supplies for the amplifiers; (6) storage unit; (7) data acquisition. In order to transmit the acoustic waves from the sensor to the material, a water column $h=60 \mathrm{~mm}$ was maintained during the testing (see Figure 5.11a). The pipe was rotated such that every tested area is placed at the bottom of the pipe. Figure 5.11c depicts the starting point location and position of the aluminium carrier together with the sensors.

For the measurements that were performed at the same location (field pipe) the extracted parameters were averaged. Figure 5.12 shows a typical power spectrum from the measurements on the field pipe. The three main components of the spectrum are shown in green, blue and red colours for the $f_{2}, f_{1}$ and $f_{g}$ frequencies respectively. The maximum of each component is extracted. 

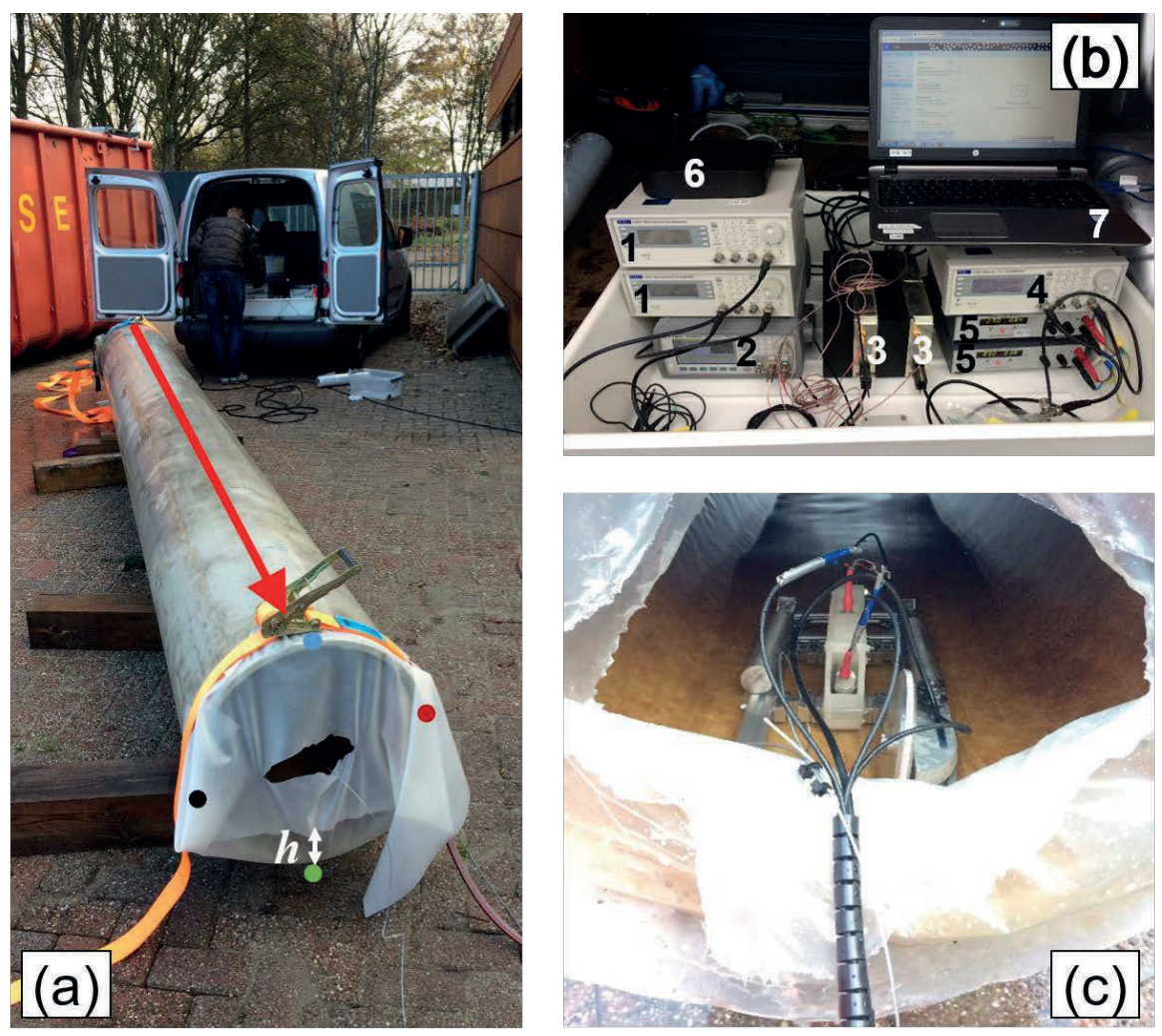

Figure 5.11

Field test set-up showing (a) the inspected pipe with the 4 sections inspected: $0^{\circ}$ (blue), $90^{\circ}$ (red), $180^{\circ}$ (green) and $270^{\circ}$ (black); (b) the electronics; (c) the carrier with the sensors and the cables together inside the pipe.

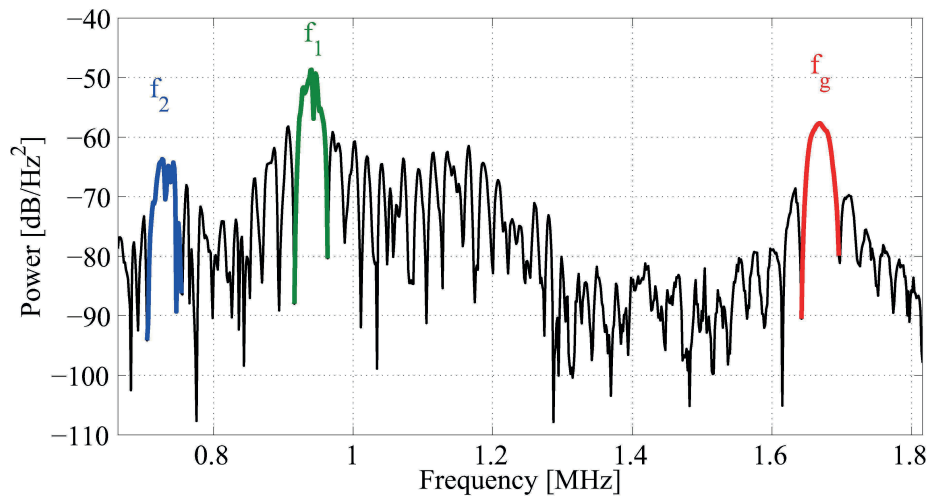

Figure 5.12

Frequency spectrum from the area at $180^{\circ}$ from the field PVC pipe test. 


\subsection{Results and discussion}

The results are divided in four sections. In the first section, the amplitude from the generated wave is compared between one-sided and two-sided configuration in the laboratory samples. Lastly, the measured amplitudes on the field pipe experiments are shown.

\subsubsection{Non-collinear wave mixing laboratory tests}

The distance that the generated wave has to travel in the material is longer for a one-sided configuration than a two-sided configuration (as depicted in Figure 5.7). Thus, the latter has less attenuation. The generated wave from both experiments (one-sided and two-sided) can be detected with a $27 \mathrm{~dB}$ and a $30 \mathrm{~dB}$ signalto-noise ratio. The signal level in the one-sided configuration is not significantly lower than the attenuation from that of the two-sided configuration making it a feasible measurement technique.

The power spectrum amplitude for each specimen is shown in Figure 5.13. The amplitude from the two-sided experiments is higher than the one for the one-sided. This behaviour is expected due to the longer distance travelled by the generated wave in the one-sided configuration (see Figure 5.13a). The amplitudes of the 25802-PR specimen are fairly the same. The interaction in this case happened rather at a distance closer to the front surface, while the interaction in the other two cases happened at a deeper distance from the front surface. The amplitudes of the pump wave's frequency components remain at a higher amplitude for the double-sided experiments (see Figures 5.13b and 5.13c).
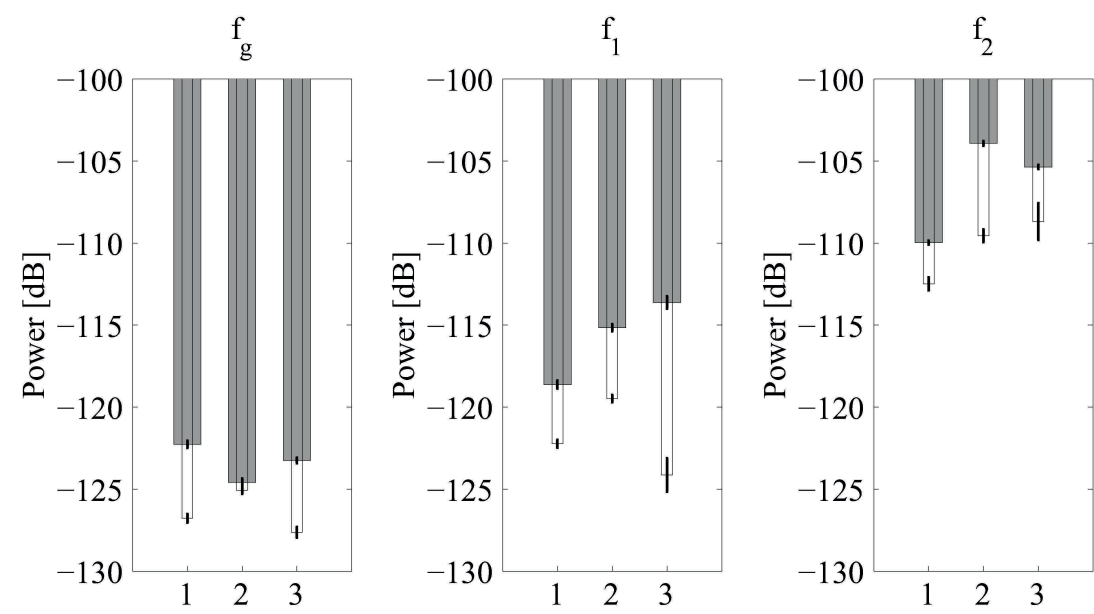

Figure 5.13

Amplitude of the (a) generated wave; (b) pump wave one; (c) pump wave two. White box plot stands for the one-sided experiments and grey box plot stands for the two-sided configuration. 
The positioning of the sensors is the major contributor the recording higher energy from the pump wave's and it is not an effect attributed to the two-sided configuration. For instance, lower energy from the two-sided experiment can be recorded from a different interaction configuration where the receiver should be located at a larger distance from the path of the incident beams.

The difference between the two scenarios in terms of the signal strength is comparable. The detection of ageing in a PVC specimen was performed in a twosided configuration in previous research [10]. Thus, here it is shown that ageing in PVC can also be measured in a one-sided configuration. The signal strength shown in this research is a fundamental step for the implementation of such a technique for the inspection of PVC pipes in a one-sided configuration.

The SNR for each specimen type for the one-sided and two-sided is shown in Table 5.7. A maximum of $4 \mathrm{~dB}$ of difference between the one-sided and twosided experiments was found. The SNR of the generated wave in the onesided experiment is comparable with the SNR of the two-sided configuration.

Table 5.7 Signal to noise ratio (SNR) of the generated wave

\begin{tabular}{|c|c|c|c|}
\hline & $\begin{array}{c}S N R_{1-\text { sided }} \\
{[\mathrm{dB}]}\end{array}$ & $\begin{array}{c}S N R_{2 \text {-sided }} \\
{[\mathrm{dB}]}\end{array}$ & $\begin{array}{c}P_{2 \text {-sided }}-P_{1 \text {-sided }} \\
{[\mathrm{dB}]}\end{array}$ \\
\cline { 2 - 4 } 1-6704-PR & 27 & 31 & -4 \\
2-5802-PR & 28 & 29 & -1 \\
\cline { 2 - 4 } 3-58-02-IM & 26 & 30 & -4 \\
\cline { 2 - 4 } & &
\end{tabular}

\subsubsection{Testing of PVC pipe}

The signal-to-noise ratio is shown in Figure 5.14 at each measurement location. From the four inspected sections, the section at $0^{\circ}$ has the highest power spectrum. The amplitude of the power spectrum of the generated wave is of greater amplitude compared to the one from the pump wave frequency component two (see Figure 5.14a). For section $0^{\circ}$, the scattering of the power spectrum of the frequency components $f_{1}=2.67 \mathrm{~dB}$ and $f_{2}=4.38 \mathrm{~dB}$.

The scatter of the power spectrum for the section 90 are $f_{1}=5.36 \mathrm{~dB}, f_{2}=4 \mathrm{~dB}$. The amplitude of the power spectrum of the pump wave one and two, for the inspected sections $0^{\circ}$ and $90^{\circ}$ (Figure 5.14a and 5.14b respectively), are fairly the same. However, the power spectrum of the generated wave for the inspected section at $90^{\circ}$ is approximately $13.72 \mathrm{~dB}$ much lower than the one from the $0^{\circ}$ section.

The amplitude of the power spectrum of the pump wave one and two, for the inspected sections $0^{\circ}$ and $180^{\circ}$ are different. This difference is of $5 \mathrm{~dB}$. Furthermore, the power spectrum of the generated wave for the inspected section at $180^{\circ}$ is approximately $15.4 \mathrm{~dB}$ lower than the one from the $0^{\circ}$ section. The 
scattering of the power spectrum pump waves for the section $180^{\circ}$ are $f_{1}=14.45$ $\mathrm{dB}$ and $f_{2}=16.94 \mathrm{~dB}$.

When the power of the generated wave is lower than $-85 \mathrm{~dB}$ it can be barely detected. For the sections $90^{\circ}$ and $180^{\circ}$ only a few measurements were above this level. The noise level is at $-95 \mathrm{~dB}$ at the frequency of the generated wave. The spectrum of the generated wave for the $270^{\circ}$ was at the noise level. The power spectrum of both the pump waves for the $270^{\circ}$ section decreased $10.12 \mathrm{~dB}$ compared to the ones from the $0^{\circ}$ section.

Four main observations can be pointed out. The power spectrum of the generated wave can be of higher amplitude than the one of the second pump wave. This is due to the thicker thickness of the specimen compared to the ones from the laboratory which allows the beam path of the incident waves to diverge far from the location of the receiver (see Figure 5.7). Additionally, the interaction volume in a thicker specimen is higher compared to the thinner specimens [19]. Furthermore, more energy is converted to a generated wave compared to locations were the power of the second pump wave component is higher than the power of the generated wave.

The second observation is that the generated wave's power spectrum decreases when the scattering of the pump wave's amplitude spectrum increases as shown in Figure 5.14b, Figure 5.14c and Figure 5.15. Even a minimal increase in the standard deviation from the two pump waves $(2.68 \mathrm{~dB})$ has a negative effect of $13.72 \mathrm{~dB}$ in power loss in the generated wave (see Figure 5.15). A higher increase in standard deviation $(11.77 \mathrm{~dB}$ ) reduces the power of the generated wave to 15.4 $\mathrm{dB}$. The main reason for this is that in the measurement locations where there is an increase in the standard deviation the incident waves reflect in other directions and thus, the generation of the wave is less effective. This effect is both seen in the generated wave as well as in the pump waves.

Thirdly, the complete loss of generated wave's amplitude is correlated with a decrease of $10.12 \mathrm{~dB}$ in the pump wave's power spectrum amplitude and a decrease in the scattering of the measurements (see Figure 5.15). This means that only the reflection from the first surface of the specimen is captured by the receiver and not the refracted energy inside the PVC. The latter beam path diverges to directions where it is not possible to generate a wave and neither return to the receiver. This is the worst-case scenario where there is no generation of a wave at all.

Lastly, the maximum SNR found in the fourth section inspected is of $30 \mathrm{~dB}$ as shown in Figure 5.14a. However, the average SNR from locations shown in Figure $5.14 \mathrm{~b}$ and $\mathrm{c}$ is between $10 \mathrm{~dB}$ and $20 \mathrm{~dB}$ for all the measurements. When proper alignment has been achieved between the sensors and the specimen a signal strength with $30 \mathrm{~dB}$ SNR can be achieved. 


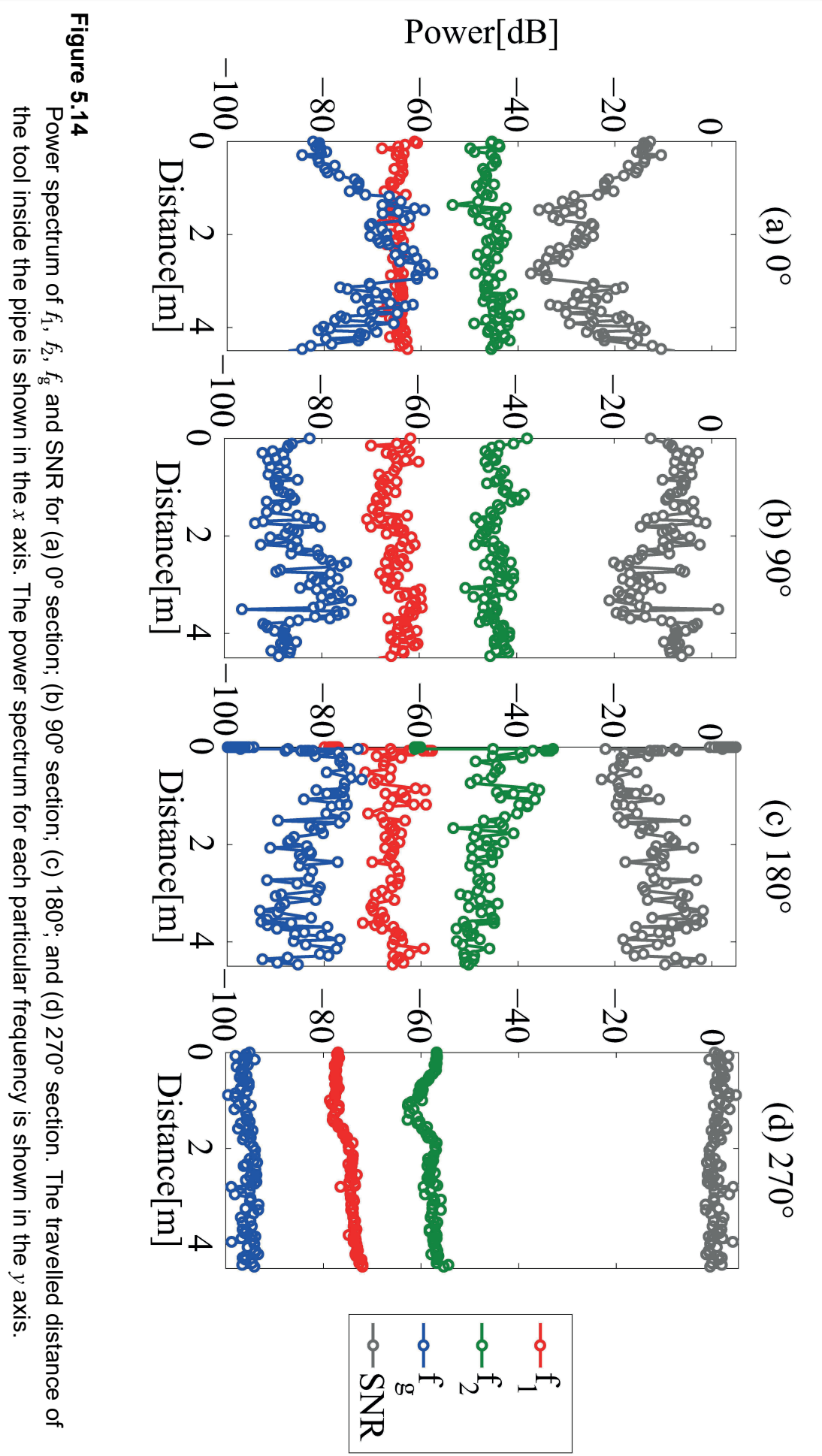






Figure 5.15

Standard deviation of $f_{1}$ and $f_{2}$ and power of $f_{g}$ for the 4 inspected areas

In comparing the results between the laboratory and the field specimens, the difference in SNR between these two (when proper alignment has been achieved) is $4 \mathrm{~dB}$. These results are exceptional as the thickness of the pipe is four times thicker than the laboratory specimens. The method can be perfectly used in field inspections.

\subsection{Conclusions}

In this research, three different types of PVC samples were tested with the wave mixing technique in a one-sided and in a two-sided wave mixing configurations. Thereafter, a PVC pipe taken from the field was tested in a one-sided wave mixing configuration. The following conclusions are drawn:

- It is possible to use the wave mixing technique for the inspection of PVC pipes in a one-sided configuration;

- The presented methodology to estimate the shear wave velocity is very sensitive to errors in the measurements;

- Optimization of the generated wave amplitude is done by adjusting the pump wave frequencies for each type of material;

- The errors in the measured shear wave velocity are compensated by frequency optimization until the maximum amplitude of the generated wave is reached. This method can be used without knowing accurately the shear wave velocity, making it robust to be used where not all the parameters of the material under test are known;

- The signal-to-noise ratio in the one-sided configuration is similar to the one in the two-sided configuration;

- The signal strength of the field tests is comparable to the ones from the laboratory; 


\section{ACKNOWLEDGMENT}

This work was performed in the cooperation framework of Wetsus, European Centre of Excellence for Sustainable Water Technology (www.wetsus.eu). Wetsus is co-funded by the Dutch Ministry of Economic Affairs and Ministry of Infrastructure and Environment, the Province of Fryslân, and the Northern Netherlands Provinces. The authors like to thank the participants of the research theme "Smart Water Grids" for the fruitful discussions and their financial support. The authors thank to Evides Waterbedrijf for providing the PVC pipe and to Wavin for providing the PVC samples. 


\section{REFERENCES}

1. M. A. Christie and B. Phelps, "Fatigue testing of uPVC pressure pipe," Int. J. Fatigue, vol. 6, no. 2, pp. 125-127, 1984.

2. H. A. Visser, L. L. Warnet, and R. Akkerman, "An attempt to use scratch tests to predict the residual lifetime of unplasticised poly(vinyl chloride) pipes," Eng. Fract. Mech., vol. 76, no. 18, pp. 2698-2710, 2009.

3. H. A. Visser, "Residual lifetime assessment of uPVC gas pipes," University of Twente, Enschede, The Netherlands, 2010.

4. E. Kuliczkowska and A. Zwierzchowska, "A qualitative analysis of early defects present in PVC-U sewers but not observed in rigid pipes," Tunn. Undergr. Sp. Technol., vol. 56, pp. 202-210, 2016.

5. S. J. F. Gould, P. Davis, D. J. Beale, and D. R. Marlow, "Failure analysis of a PVC sewer pipeline by fractography and materials characterization," Eng. Fail. Anal., vol. 34, pp. 41-50, 2013.

6. W. Guo, L. Soibelman, and J. H. Garrett, "Automated defect detection for sewer pipeline inspection and condition assessment," Autom. Constr., vol. 18, no. 5, pp. 587-596, 2009.

7. M. R. Halfawy and J. Hengmeechai, "Automated defect detection in sewer closed circuit television images using histograms of oriented gradients and support vector machine," Autom. Constr., vol. 38, pp. 1-13, 2014.

8. Z. Liu and Y. Kleiner, "State of the art review of inspection technologies for condition assessment of water pipes," Meas. J. Int. Meas. Confed., vol. 46, no. 1, pp. 1-15, 2013.

9. M. Lepot, N. Stanić, and F. H. L. R. Clemens, "A technology for sewer pipe inspection (Part 2): Experimental assessment of a new laser profiler for sewer defect detection and quantification," Autom. Constr., vol. 73, pp. 1-11, 2017.

10. A. Demčenko, R. Akkerman, P. B. Nagy, and R. Loendersloot, "Non-collinear wave mixing for non-linear ultrasonic detection of physical ageing in PVC," NDT E Int., vol. 49, pp. 34-39, 2012.

11. L. Laiarinandrasana, E. Gaudichet, S. Oberti, and C. Devilliers, "Effects of aging on the creep behaviour and residual lifetime assessment of polyvinyl chloride (PVC) pipes," Int. J. Press. Vessel. Pip., vol. 88, no. 2-3, pp. 99-108, 2011.

12. J. M. Hutchinson, "Physical aging of polymers," Prog. Polym. Sci., vol. 20, no. 4, pp. 703-760, Jan. 1995.

13. E. Drenth, "Residual lifetime of uPVC pipes," University of Twente, Enschede, The Netherlands, 2015.

14. D. Walton and W. J. Elzink, "The long term behaviour of buried uPVC sewer pipe,” Constr. Build. Mater., vol. 3, no. 2, pp. 58-63, 1989.

15. R. K. Walter, P. H. Lin, M. Edwards, and R. E. Richardson, "Investigation of factors affecting the accumulation of vinyl chloride in polyvinyl chloride piping used in drinking water distribution systems," Water Res., vol. 45, no. 8, pp. 2607-2615, 2011.

16. J. A. Kwon and R. W. Truss, "The work of fracture in uniaxial and biaxial oriented unplasticised polyvinylchloride pipes," Eng. Fract. Mech., vol. 69, no. 5, pp. 605-616, 2002. 
17. D. B. West and R. W. Truss, "Mode II delamination testing in uniaxially oriented PVC pipes," J. Mater. Sci., vol. 39, no. 8, pp. 2789-2794, 2004.

18. H. H. Delgadillo, R. Loendersloot, D. Yntema, T. Tinga, and R. Akkerman, "Experimental Validation of Non-Collinear Wave Mixing Model in a PVC Specimen," in 2018 IEEE International Ultrasonics Symposium (IUS), 2018, vol. 107, no. December, pp. 1-9.

19. H. Hernandez Delgadillo, R. Loendersloot, D. Yntema, T. Tinga, and R. Akkerman, "Steering the propagation direction of a non-linear acoustic wave in a solid material," Ultrasonics, vol. 98, no. April, pp. 28-34, 2019.

20. G. L. Jones and D. R. Kobett, "Interaction of Elastic Waves in an Isotropic Solid," J. Acoust. Soc. Am., vol. 35, no. 1, pp. 5-10, 1963.

21. A. J. Croxford, P. D. Wilcox, B. W. Drinkwater, and P. B. Nagy, "The use of non-collinear mixing for nonlinear ultrasonic detection of plasticity and fatigue.," J. Acoust. Soc. Am., vol. 126, no. 5, pp. EL117-L122, 2009.

22. B. M. Lempriere, "Wave Propagation Concepts," in Ultrasound and Elastic Waves, Elsevier, 2003, pp. 25-44.

23. F. Irgens, "Theory of Elasticity," in Continuum Mechanics, Berlin, Heidelberg: Springer Berlin Heidelberg, 2008, pp. 199-302.

24. V. A. Korneev and A. Demčenko, "Possible second-order nonlinear interactions of plane waves in an elastic solid," J. Acoust. Soc. Am., vol. 135, no. 2, pp. 591-598, 2014.

25. E. Escobar-Ruiz, A. Ruiz, W. Hassan, D. C. Wright, I. J. Collison, P. Cawley, and P. B. Nagy, "Non-linear ultrasonic NDE of titanium diffusion bonds," J. Nondestruct. Eval., vol. 33, no. 2, pp. 187-195, 2014. 



\section{Chapter}

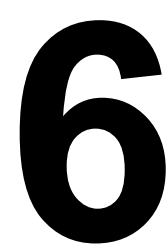

\section{General Discussion}

In this chapter the knowledge gained in the previous chapters is integrated into two generalized flow charts indicating the major steps and variables to consider for developing inspection techniques (not limited to the drinking water sector). Additionally, in this chapter the status of the inspection methodologies at the end of this project is described. Finally, some ideas on how the drinking water network should be monitored are discussed. 


\subsection{Initial problem overview}

The drinking water supply in the Netherlands has been in operation for almost a century. The infrastructure that distributes drinking water has been undergoing continuous degradation, but the precise state of the pipelines is unknown. Monitoring the condition of the water network can enhance the management of assets and is required so as to establish an adequate replacement plan. Therefore, an inspection method is required which is capable of detecting and estimating degradation levels in the pipes. In this thesis, the use of ultrasonic sensors for the inspection of drinking water pipes in an inline configuration setting was studied. Approximately $50 \%$ of the network is made of PVC and approximately $30 \%$ is made of cementitious materials. Only these two materials were studied. In this final chapter, the results are discussed from a more integral view. The current status of the developments is sketched and some ideas on how the drinking water network could be maintained in the future are discussed.

\subsection{Development of ultrasonic inspection methods for the drinking water pipes}

The integration of the knowledge produced throughout this thesis is organized in two generalized diagrams shown in Figure 6.1 and Figure 6.2. The flow chart in Figure 6.1 gives an overview of the essential variables involved when developing inspection techniques for drinking water pipes, whereas the flow chart in Figure 6.2 represents the steps of translating raw measurement data into degradation levels. Note that the steps in these two flow charts can easily be generalized and applied to other types of materials and structures. However, it should be kept in mind that the decision to commence the development of an inspection technique depends on many factors. These include the maintenance policies of the utilities, finance, maintenance techniques and the cost-benefit of developing condition monitoring and inspection methods [1]. In Chapter 2 of this thesis, the two main degradation mechanisms present in cement-based drinking water pipes were identified (block 2 in Figure 6.1) and the respective features of the selected nondestructive method were found sensitive to these two mechanisms (block 3 to block 5).

In Chapter 3 it was demonstrated that it is possible to use this technique in a real inline inspection (from block 6 to block 7 in Figure 6.1) and to use it for the condition evaluation of $A C$ pipes. This inspection technique is presented in block 8 (green oval in Figure 6.1). Block 7 is described in more detail in the flow chart in Figure 6.2.

For PVC pipes, the starting point is indicated with the red box ('No') in Figure 6.1. This means that the earlier work at The University of Twente by Roy Visser [2] (block 2), Emiel Drenth [3] (block 2) and Andriejus Demčenko [4] (block 3, 4 and 5) have built up the schematic process (Figure 6.1) up to block 6. Upscaling of the selected NDT method seemed very challenging. The breakthroughs in Chapters 


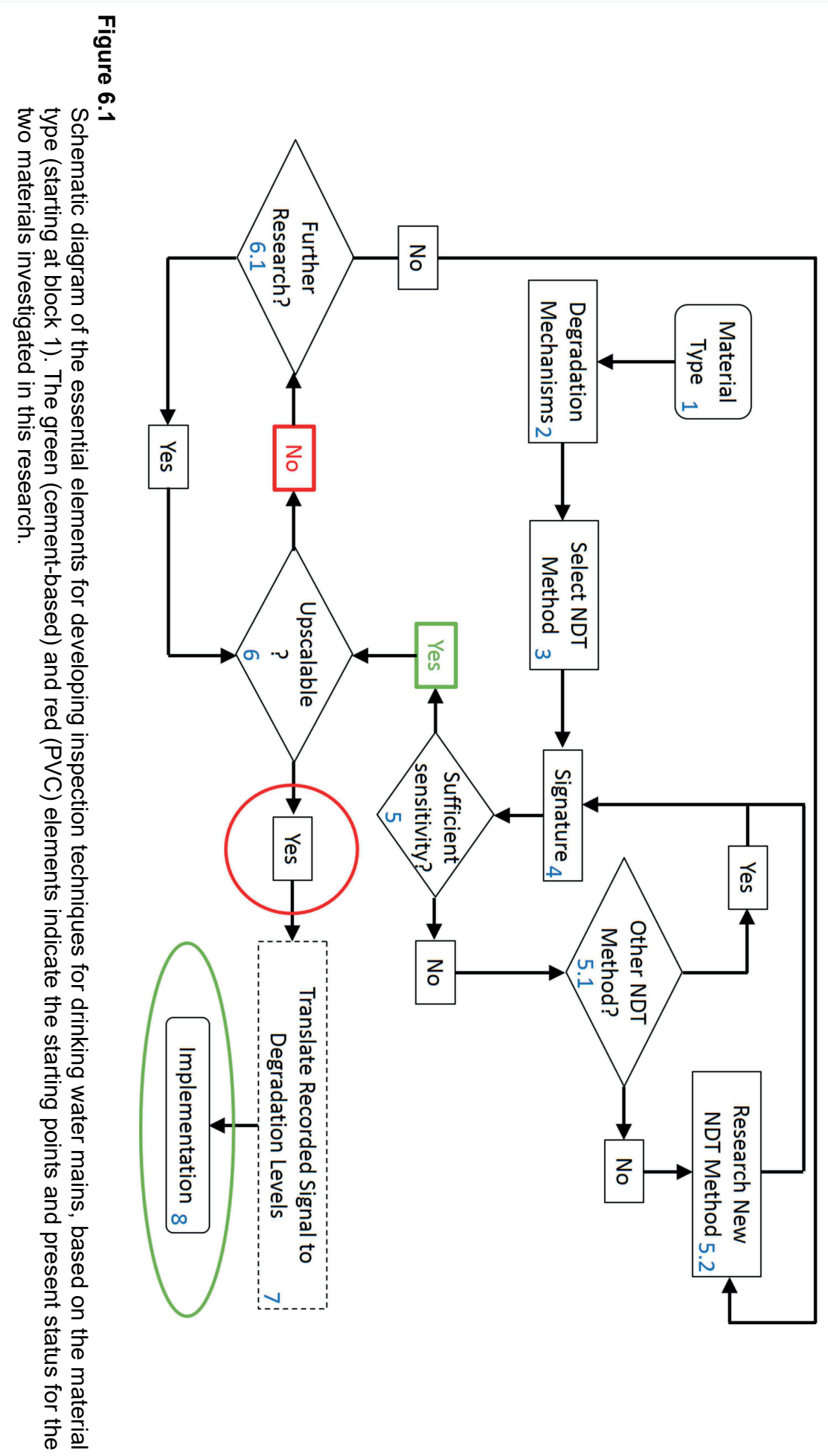


4 and 5 drove the wave mixing technique forward to a greater maturity for the inspection of PVC drinking water pipes. The inspection technique for PVC pipes is shown in block 7 (red circle in Figure 6.1).

In developing inspection methodologies, some questions arise that are not very straightforward to answer, as for example block 6 in Figure 6.1. Performing measurements in a controlled environment such as the laboratory will not provide the information necessary to upscale a technology. An advantage of the Wetsus 'Smart Water Grids' platform is the close collaboration with industrial partners. This allowed for relatively quick field test arrangements for both AC (chapter 3 ) and PVC (chapter 5).

To guarantee broad applicability, the process was described as generically as possible. Consequently, it still is a good guideline for the relevant milestones in developing an inspection technique.

The second generalized process is the translation of raw data into useful information on the degradation level as depicted in Figure 6.2. This flow chart is a detailed representation of block 7 in Figure 6.1. This schematic was created after the model developed in Chapter 2 to estimate the degradation levels. In Chapter 3 , this model was used in a real case scenario. The steps shown in Figure 6.2 can be used to translate the signal parameter from the wave mixing testing (in PVC) into degradation levels, in a similar way to what has been done with AC. The same steps can be followed to obtain useful information from the raw measurement data for other inspection techniques as well.

Approximately $20 \%$ of the drinking water network is assembled using materials other than PVC or AC. These are polyethylene (PE), cast iron, steel and glass fibre-reinforced plastics (GFRP). For some of these distribution pipes there is insufficient knowledge with respect to the degradation mechanisms, e.g. for glass fibre-reinforced plastics. If the cost-benefit analysis of developing an inspection technique for GFRP pipe reports that the maintenance of the pipe should be preventive [5], the tools shown in Figure 6.1 and Figure 6.2 can guide this development into the right direction. For instance, the degradation mechanisms should firstly be examined, followed by selecting and evaluating an NDT method that is sensitive enough to detect the main degradation mechanisms. The processes developed in this chapter highlight the key milestones to achieve in order to develop an inspection technique. 


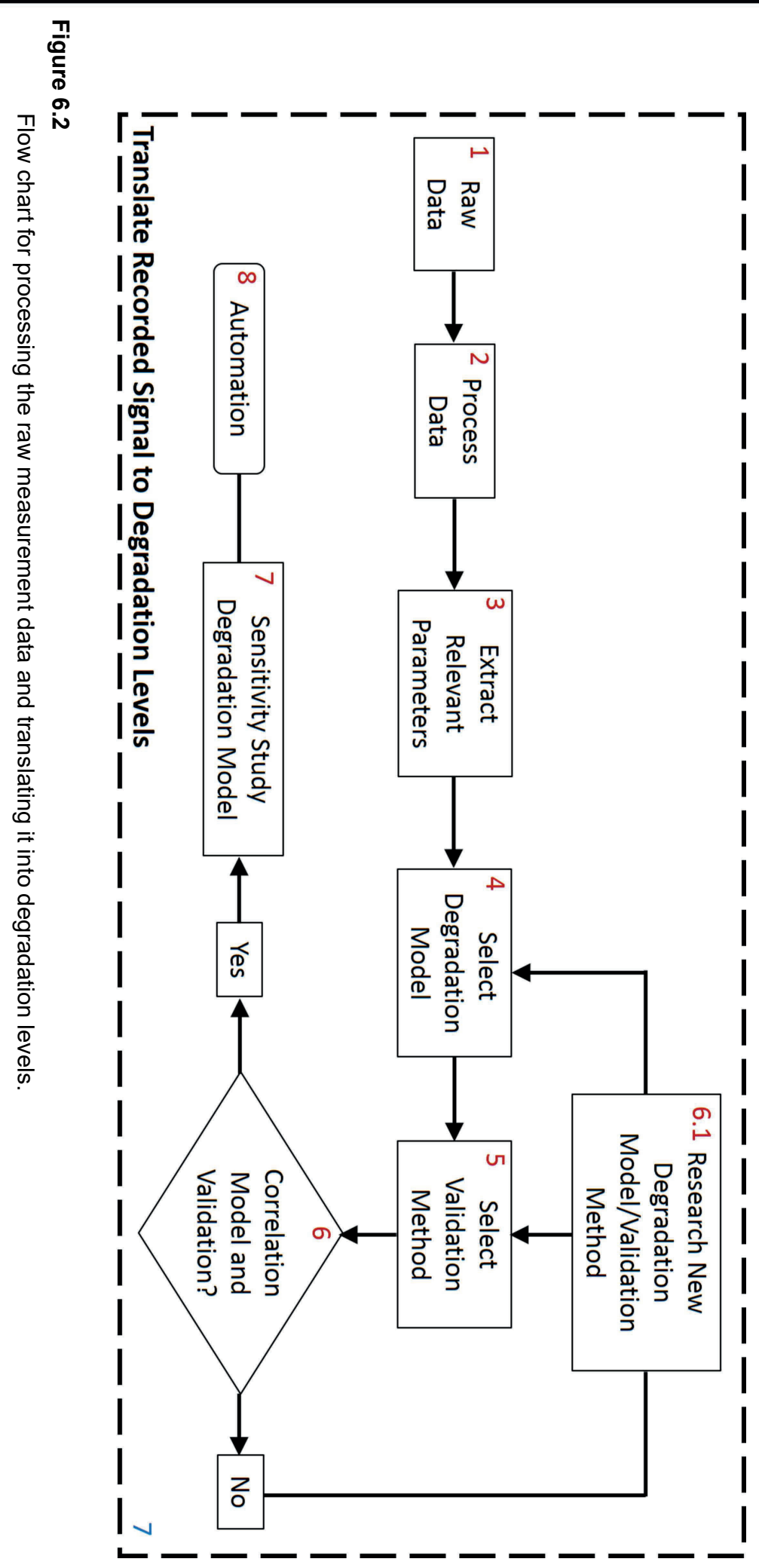




\subsection{Summary on achieved research objectives}

In the introduction of this thesis, the research aim was defined as 'Development of an ultrasonic testing method which is capable of detecting and measuring the degradation of a water main section' and 'Upscaling the ultrasonic method from laboratory scale to inline inspection of water main sections'. Several research questions were defined to guide the research. In this section, these research questions are reviewed and the extent to which they have been answered in this work for both $A C$ and PVC is discussed.

1. How can the degradation state of water mains be determined from ultrasonic measurement data?

a. What is the relationship between measurement results and the test settings?

b. How can a measure for the level of degradation be obtained from the ultrasonic signal?

AC: In Chapter 2, the relationship between the test settings and measurement results was characterized. In addition, in Chapter 2, the translation from ultrasonic measurement to degradation level was developed based on pulse velocity measurements. The range of the detected degradation levels was found to be primarily a function of the central frequency of the piezoelectric material in the transmitter/receiver. Based on this, the optimal frequency for different pipe wall thicknesses can be selected.

PVC: Research question 1a had partly been answered previously by Demčenko for PVC et al. [4]. Research question 1.a was addressed partly in Chapter 4 and Chapter 5. The major factors to consider for the amplitude of the wave mixing technique are the frequencies of the pump waves and the positioning of the sensors.

2. What are the relevant factors to consider for the transition from laboratory conditions to field conditions?

a. How to move from a two-sided to a one-sided approach?

b. How can a variation in degradation in the circumferential direction and in the longitudinal direction be determined?

AC: For cement-based pipes, it was found in Chapter 3 that in a real pipe inspection the factors that have a relevant effect on the estimated degraded depth are the speed of sound in pristine condition, the speed of sound in fully degraded cement and the pipe thickness. Other factors that have an effect on the ultrasonic data are the pipe-soil interaction, the water flow and temperature. Neither of the latter two seems to have a significant effect on the ultrasonic signals. In the cement-water interface, the energy transmitted to the water is higher than the energy transmitted to the soil in the pipe-soil interface. Hence, a dry soil contributes positively to the signal strength of the reflected signal. The temperature has an effect on the measured, but this was not studied in this thesis. 
Research question 2 was therefore answered, although further research should focus on the effect of temperature on the measured degradation levels.

Degradation appears to be present in the cementitious pipes in a consistent manner along the longitudinal direction. In the circumferential direction, fluctuations in degradation levels seem to be present only in some parts of the network. Considering solely these results, the presence of degradation with the revealed patterns is not yet certain. Thus, field verification checks of the estimated acoustic-based degraded depth have to be performed.

PVC: considering the complexity of the wave mixing technique for the detection of ageing in PVC, a different route was taken. The efforts were targeted to advance the two-sided wave mixing technique from the laboratory to the real-life conditions in spite of the lack of a translation of the acoustic parameters into degradation levels. This is because of two major drawbacks of the wave mixing technique. The first is associated with the acoustic channel: With slight deviations in the test configurations the amplitude of the generated wave diminishes. The amplitude of the generated wave carries the information on the degradation levels of PVC (physical ageing). It is shown in Chapter 4 that the generated wave could be still captured by the receiver by changing the frequencies of the incident waves in case a deviation occurs during an inline inspection (misalignment for instance). The second is that even in a two-sided wave mixing configuration the amplitude of the generated wave is low compared to for instance the amplitude strength of a pulse echo configuration. This raised the question whether the signal strength in a single sided access configuration, which is necessary in field applications, would be sufficiently strong. In chapter 5 it was shown that a one-sided wave mixing configuration has a similar signal strength as that with a two-sided configuration. With this second issue addressed, the technology is brought again a step closer to the field application. The final step is to characterize the factors that influence the estimation of ageing levels.

\subsection{Outlook on inspection technologies and management of the drinking water network}

Cementitious drinking water pipes that are currently in service are now being inspected by a member of the consortium as a result of this research. Yet a major challenge remains: the thickness of installed AC pipes is unknown. It is recommended that the assumed thickness and the estimated degraded depth be validated with destructive testing methods (e.g. phenolphthalein or CT scans). The speed of sound in pristine and degraded condition can be calibrated with these destructive testing methods. This is currently a topic of research by the Dutch drinking water utilities.

Other types of indications can be found in the measurement data, such as joint misalignment, internal diameter variations and material changes. Areas with relatively high degradation and other types of indications can then be located in the network, allowing the water companies to take actions accordingly. An 
example of this was shown in Chapter 3, where the pipe sections with the highest estimated degradation levels were located next to farm buildings. Within cities this might be more complicated; however, it seems to be possible to correlate the type of urban area with degradation levels.

For PVC pipes, the main limitation to estimating the degradation levels is the lack of a model that relates the nonlinear coefficient to the material state. Therefore, $a$ second technique to determine the degradation levels has to be selected in order to validate the method that estimates the ageing in PVC. Some techniques found in literature that could be suitable for this are micro-hardness, creep tests and differential scanning calorimetry (DSC) $[5,6]$.

Because the wave mixing technique is not a very well-known technology, the findings in Chapter 4 have caused this research to be expanded to sensor technologies. An example of this is a recently started PhD project within the smart water grids research theme. Larger areas can be covered with phased array sensors, reducing the complexity of a test configuration even more. Hence, the wave mixing technique can potentially be used for imaging the pipe thickness from the inside as a function of ageing. The wave mixing could also be used to measure fatigue damage in cast iron and stainless-steel pipes, as it has been for other metals [7-9].

Ultrasonic techniques were developed for different materials, yet relying on the same sensors. There is even potential for using these ultrasonic techniques, or slightly adapted ones, for other pipe materials. This implies that the entire water distribution network, from a minimum pipe diameter, can be inspected with only one inspection tool, which is a great advantage for inspection companies such as Acquaint B.V. as well as for the water companies, as inspection costs will reduce.

The scope of this thesis does not encompass the problem of deciding which pipes should be inspected first and which pipes should be replaced first. Nonetheless, within the Smart Water Grids research theme, two recently started research projects will engage in providing some answer to these issues. One of them (Smart detection and real-time learning in water distribution: an integrated datamodel) explores a data-driven early warning system based on abnormal behaviour of pressure valves and recorded failure events [11]. With this type of systems early action protocols could be developed that include changing the network use or performing routine inspections. The second project (Smart-pipes, monitoring assets for efficient maintenance) is the most recently started project and focuses on embedded sensors within the network. Similarly, the data from these sensors could trigger pipe inspection or pipe replacement.

In the long term, the water utilities should strive towards a combination of datadriven and prediction models. Failure of pipes can be reduced by means of monitoring the condition of the pipes and monitoring the parameters relevant to the prediction models $[12,13]$. The use of models should in the long term replace the use of inspection technologies to a large extent. 


\section{REFERENCES}

1. W. Tiddens, 'Setting sail towards predictive maintenance: developing tools to conquer difficulties in the implementation of maintenance analytics,' University of Twente, Enschede, The Netherlands, 2018.

2. H. A. Visser, 'Residual lifetime assessment of uPVC gas pipes,' University of Twente, Enschede, The Netherlands, 2010.

3. E. Drenth, 'Residual lifetime of uPVC pipes,' University of Twente, Enschede, The Netherlands, 2015.

4. A. Demcenko, 'Development and analysis of noncollinear wave mixing techniques for material properties evaluation using immersion ultrasonics,' Cambridge University Press, Enschede, The Netherlands, 2014.

5. J. Pieters, 'Insurer pays out $€ 34.8$ million to VU Hospital for water damage caused by water main break,' NLTimes, 2016. [Online]. Available: https://nltimes.nl/2016/09/08/insurer-pays-eu348-million-vu-hospital-waterdamage-caused-water-main-break.

6. L. Laiarinandrasana, E. Gaudichet, S. Oberti, and C. Devilliers, 'Effects of aging on the creep behaviour and residual lifetime assessment of polyvinyl chloride (PVC) pipes,' Int. J. Press. Vessel. Pip., vol. 88, no. 2-3, pp. 99-108, 2011.

7. J. M. Hutchinson, 'Physical aging of polymers,' Prog. Polym. Sci., vol. 20, no. 4, pp. 703-760, Jan. 1995.

8. A. J. Croxford, P. D. Wilcox, B. W. Drinkwater, and P. B. Nagy, 'The use of non-collinear mixing for nonlinear ultrasonic detection of plasticity and fatigue.,' J. Acoust. Soc. Am., vol. 126, no. 5, pp. EL117-L122, 2009.

9. A. J. Croxford, P. D. Wilcox, and B. W. Drinkwater, 'Non-linear material characterisation using the noncollinear method,' Nondestruct. Charact. Compos. Mater. Aerosp. Eng. Civ. Infrastructure, Homel. Secur. 2010, vol. 7649, no. April 2010, p. 76491X, 2010.

10. G. Tang, M. Liu, L. J. Jacobs, and J. Qu, 'Detecting localized plastic strain by a scanning collinear wave mixing method,' J. Nondestruct. Eval., vol. 33, no. 2, pp. 196-204, 2014.

11. C.V. C. Geelen, D. R. Yntema, J. Molenaar, and K. J. Keesman, 'Monitoring Support for Water Distribution Systems based on Pressure Sensor Data,' Water Resour. Manag., Jul. 2019.

12. T. Tinga and R. Loendersloot, 'Physical Model-Based Prognostics and Health Monitoring to Enable Predictive Maintenance,' in Predictive Maintenance in Dynamic Systems, Cham: Springer International Publishing, 2019, pp. 313353.

13. B. Wols, A. Moerman, P. Horst, and K. van Laarhoven, 'Prediction of Pipe Failure in Drinking Water Distribution Networks by Comsima,' Proceedings, vol. 2, no. 11, p. 589, 2018. 



\section{Chapter}

\section{Conclusions and recommendations}

In this thesis, non-destructive methods for inspecting the drinking water pipes based on ultrasonic sensors were studied. In this final chapter, the overall conclusions are drawn. Some practical and scientific recommendations are given as well. 


\subsection{Conclusions}

Based on the objectives and research questions established at the beginning of this thesis, the following conclusions are drawn. The conclusions are generalized for the development of inspection methods for any type of material but examples are given for the specific materials investigated in this thesis.

- Based on the objectives and research questions established at the beginning of this thesis, the following conclusions are drawn. The conclusions are generalized for the development of inspection methods for any type of material but examples are given for the specific materials investigated in this thesis.

- The degradation mechanism relevant to the type of material is the driver for selecting an ultrasonic testing method. For example, in cement-based pipes the degradation mechanism has a direct effect on the linear elastic properties, hence linear ultrasonics was identified as a feasible method for proceeding further with the inspection method;

- The degradation levels can be estimated from relating an acoustic measurement parameter to a physics-based model. The degradation levels in cement-based pipes were estimated based on the acoustic impedance of the materials and the pulse velocity. For the PVC pipes, the ageing levels can be determined from relating the acoustic parameter (e.g. amplitude of the generated wave) to a mechanical property (e.g. creep behaviour).

- The degradation patterns (extent of degradation through the longitudinal and circumferential direction of the pipe) in the drinking water network are present along the circumference of the pipe and in the longitudinal direction. These are determined based on the estimated degradation levels and with the aid of an inspection tool. An example of this is the degradation pattern discovered in cement-based pipes.

- The relevant factors that have an effect on the measured condition in a pipe section are the characteristics of the pipe itself (pristine conditions and thickness). The assumption that the pipe wall thickness is constant throughout an inspection is valid. Nevertheless, determining the true pipe wall thickness remains a challenge for installed pipes

- $\quad$ Field application aspects can be advanced while laboratory developments are in progress. A clear example of this is the upgrade of the wave mixing settings prior to correlating ageing levels to the ultrasonic signal. The state-of-the art wave mixing technique is in an advanced stage such that the measurement system can be used in a field environment. Processing the ultrasonic signals into ageing levels remains a topic of research as well as the factors that have a potential influence on the measured condition.

- The generalized approach for the development of inline inspection of water mains allowed for the development of inspection technologies all based on similar ultrasonic sensors. This permits the development of a 
single inspection tool for the entire water grid; a task currently being undertaken by Wetsus 'Smart Water Grids' theme member Acquaint B.V.

\subsection{Recommendations}

A number of recommendations are given. A distinction is made between recommendations regarding the further development of the technologies and those regarding the implementation of this type of monitoring in the maintenance practice of water companies.

\subsubsection{Scientific recommendations}

Some recommendations are given to researchers in the context of continuing with the development of inspection methods. The ideas for improving the testing of PVC are given here.

- $\quad$ The ageing levels could have an effect on the energy transmitted to the aged pipe, thus the transmission and reflection energy from longitudinal and shear waves as a function of ageing levels could be characterized. As a consequence, the energy at the interaction volume could be affected.

- Another recommendation is to examine the feasibility of reducing a wave mixing set-up from a non-collinear one-sided configuration with three sensors to a collinear one-sided configuration with two sensors.

Degradation mechanisms for cementitious pipes are not limited to leaching or acidic attack. In sewage pipes, the main degradation mechanism is a combination of carbonation and sulfuric attack. The pulse velocity method is suitable for such measurements. Here it is suggested that each degradation mechanism be characterized separately, followed by the effect of the combined degradation in the ultrasonic measurements.

\subsubsection{General recommendations}

The following recommendations are given to the water utilities for maintaining the long-term operations of the drinking water network:

- The pipelines within the drinking water network that require urgent replacement have not yet been determined. Strategies in the maintenance policies that prioritize inspection agendas should be instituted by the water utilities. With the pertinent assets targeted for condition monitoring, the probability of failure can be determined and replacement programs can therefore be initiated. Consequently, the return on investment in developing inspection technologies will be evident.

- Generate a database with information on the pipes such as thickness, and characterization of pristine conditions (e.g. acoustic, mechanical, 
chemical). The inspections of pipes would be greatly facilitated if this information was available.

- Monitor parameters such as soil pH, humidity, water quality, landslides and temperature around the drinking water infrastructure. The accuracy of the model-based and data-driven approach can potentially improve with this input. 


\section{Acknowledgements}

I would like to thank the people who contributed somehow so that this thesis could be completed. Firstly, many thanks to my daily supervisor Doekle Yntema. From Doekle I learned how to be quick on my feet in the laboratory and make things happen. I learned from him how to manage my research project more efficiently. He was also a good listener whenever I had difficulties inside or outside Wetsus. Thanks for listening. Also, many thanks to my co-promotor, Richard Loendersloot, for his patience and his professional attitude towards supervising. I learned from him how to be objective towards my own work. I really appreciate the many discussions we had even though the majority were by skype. I would like to also thank my promotor Tiedo Tinga for his input in the research articles we wrote and for his guidance in my research during the last two years. Tiedo taught me how to be more effective in what I try to communicate when writing a research article. I also appreciate that in all our meetings Tiedo was very open and considerate towards my opinion. I would like to thank my promotor Remko Akkerman for his input during the meetings we had regarding the writing of my thesis as well as the meetings for discussing the research articles. I appreciate his sharp and very efficient feedback. A word of thanks to Roy Visser who was my University supervisor at the beginning of my PhD.

I would like to thank the people from Wetsus that directly worked with me: Jan Tuinstra (el gringo), Jan Jurjen and Ernst Panstra. You guys were always available to help me and you were always cheerful. Also, Hakan Kandemir helped me in solving difficult equations, in drinking Turkish tea and in having very deep and meaningful conversations. A thank you word to the students that I supervised: Benoit Kern, Nandini Chidhambaram and Rutger Kakes. A thanks to Caspar Geelen who helped me with the supervision of Rutger (now a $\mathrm{PhD}$ colleague) and for the compelling discussions over the smart water grids. To the DBM people: Wieger Tiddens, Laura Cordova, Annemieke Meghoe, Emilio Cervantes, Rommel and the rest of the nice people. A special acknowledgement to Debbie Zimmerman from UTwente.

A special acknowledgement to Rudy Dijkstra who is a very active, positive and entrepreneurial person. His courage and business skills made this project possible. Furthermore, a thank to the people from Acquaint B.V. who significantly contributed to this thesis: Frank van der Hulst and Ethjel Tolsma. A thank you as well to the theme members who contributed in steering the direction of the project: Vitens (Eelco Trietsch), Brabant Water (Roel Diemel), PWN (Peter Horst), Wavin (Marcel Jongedijk) and Evides (Thomas van Manen).

To all my friends and colleagues that contributed in an indirect way to this thesis. Fei Liu, the first guy I made friends with. Prashanth Kumar, the awesome and crazy office mate who invited me to play chess in my first week at Wetsus (role model). The very welcoming people: Jouke Dijkstra, Jordi Moreno, Gerwin Steen and Andrew Shamu. Yin Ye and me shared the same supervisor and that made us buddies. The movie nights with Sofia Semitsoglou. Trip to Mexico with Victor 
Torres. The party people that were always at scooters and doing crazy stuff: Joeri de Valença, Natascha Pfaff and Fabian Ruhnau. Ilse Verburg my associate business partner (met Hakan). The Jaap "Driver" Dijkshoorn who was always making me laugh with silly jokes. The endless conversations with Louis Legrand (but very meaningful) and the many volleyball games we played. Gonçalo Macedo, the team leader/ manager/ researcher/ musician. Caspar "the shark" Geelen and the great swimming lessons. Angel Estévez, Chris Schott and Ricardo "R7" Cunha my football buddies.

To my food (and party too) buddies: Gerben Breidenbach-Visser, Laurens van Dijk, Wokke Wijdeveld, Mariana Rodrigues, Catarina Simões. Sebastian, for the interesting time in the Zuidergrachtswal. The great time we spend with Karine Kiragosyan in the Dutch lessons, dancing salsa/bachata and relaxing time from the thesis writing. Not to mention my official salsa partner Rebeca Pallares. Terica "The Queen" Sinclair, I miss you mami. Maarten van de Grind always making me laugh too with his silly but smart humour. Steffen Georg never stops impressing me with his amazing stories and all the random information he can keep saved in his memory.

Shuyana Heredia, mi amiguita que siempre está feliz y que me invita a sus eventos. Raquel Barbosa, that amazing trip to Cologne with my invisible friends. Marianne Heegstra, it was a pleasure showing you my country. Stan Willems, we never saw the "Once upon a time in Mexico" movie together. My second role model Yang Lei. Thank you, Sandra Dursova, for helping me programming my Arduino. Nimmy Kovoor, my Indian friend with her funny accent, we managed to reduce the number of copies of this thesis to 100 !!. Rita Branco, thank you for your patience during this last phase of my PhD and for sharing amazing food. Thomas Prot, thank you for the amazing trip to your native village and for the many volleyball games and parties.

To the nice people I met along the way: Vinu Nair, a great guy that unfortunately had to leave from amazing Leeuwarden. Mithun Choudhury, my first international colleague at the smart water grids theme. Pamela Bukowska, thank you for the amazing times at the first Heliconweg. Sonja Willemse, I lost you, where are you? Helena van der Werff was extremely welcoming and supportive when I arrive to Leeuwarden, you are amazing. Monika Szczyrba, for the space to escape from Leeuwarden.

To my top friends from Groningen María Arroyo, Cesar Sala and Andrea Márquez, you guys gave me a space to free my mind from Leeuwarden, I will never forget those times at the "madriguera" and "het pakhuis". To my Mexican community: Paulina Sosa, Mariana Rodríguez and Lucia Hernandez. Thanks for the many Mexican lunches and pozole. Pau, the Mexican trip was on your top hits. Brigette Lee, you are my brother from another mother. Thank you for all the dinners you cooked and the help when needed (the sism!).

To my remote advisors: Victor Venegas, Wendy Galicia, Natalia Delgadillo, Ricardo Rivera and Bertin Flores. 
Le agradezco a mis padres por apoyarme incondicionalmente Esther Delgadillo y Hector H. Cruz. Esta tesis es para ustedes. A mi hermana Beatriz que siempre está para cuando la necesito (y para molestarme también) y su hijo Flavio Sosa que me hace reír. 



\section{About The Author}

Hector Hernandez was born on 1st of May 1987 in Mexico city. He did his bachelor degree in Mexico City at the Instituto Tecnologico y de Estudios Superiores de Monterrey. After graduating, he started working at NDT Global as a data analyst. Thereafter he worked in the aerospace sector for over three years. He then proceeded to do his Master of Engineering at the University of Sheffield in the United

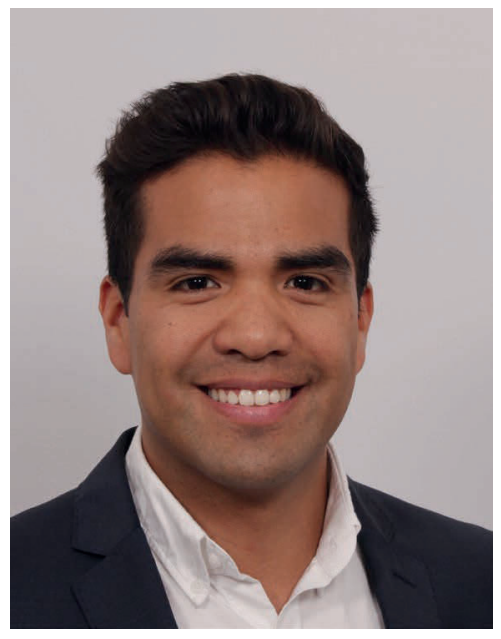
Kingdom. He did his master thesis in the Department of Aerospace Engineering where he worked on the project Extended finite element simulation of fracture initiation and propagation of laser sintered nylon 12. He successfully completed his master degree with merit. In February 2015 he started as a PhDcandidate of the University of Twente, at Wetsus in Leeuwarden 
This work was performed in the cooperation framework of Wetsus, European Centre of Excellence for Sustainable Water Technology (www.wetsus.eu). Wetsus is co-funded by the Dutch Ministry of Economic Affairs and Ministry of Infrastructure and Environment, the Province of Fryslân, and the Northern Netherlands Provinces. The authors like to thank the participants of the research theme "Smart Water Grids" for the fruitful discussions and their financial support. 